\title{
Enantioselective Synthesis of Cyclic Amides and Amines through Mo- Catalyzed Asymmetric Ring-Closing Metathesis (ARCM)
}

\author{
Elizabeth S. Sattely, ${ }^{\ddagger}$ G. Alexander Cortez, ${ }^{\ddagger}$ David C. Moebius, ${ }^{\ddagger}$ Richard R. Schrock ${ }^{\dagger}$ \\ and Amir H. Hoveyda ${ }^{\ddagger}$, \\ ${ }^{\ddagger}$ Department of Chemistry, Merkert Chemistry Center, Boston College \\ Chestnut Hill, Massachusetts 02467 \\ ${ }^{\dagger}$ Department of Chemistry, Massachusetts Institute of Technology \\ Cambridge, Massachusetts 02139
}

General: All reactions were conducted in oven- $\left(135^{\circ} \mathrm{C}\right)$ or flame-dried glassware under an inert atmosphere of dry $\mathrm{N}_{2}$. Infrared (IR) spectra were recorded on a Nicolet 210 spectrophotometer, $v_{\max }$ in $\mathrm{cm}^{-1}$. Bands are characterized as broad (br), strong (s), medium (m) or weak (w). ${ }^{1}$ H NMR spectra were recorded on a Varian Gemini 2000 (400 $\mathrm{MHz}$ ) spectrometer. Chemical shifts are reported in $\mathrm{ppm}$ from tetramethylsilane with the solvent resonance resulting from incomplete deuteration as the internal standard $\left(\mathrm{CDCl}_{3}\right.$ : $\left.\delta 7.26, \mathrm{C}_{6} \mathrm{D}_{6}: \delta 7.16,\left(\mathrm{CD}_{3}\right)_{2} \mathrm{SO}: \delta 2.50\right)$. Data are reported as follows: chemical shift, multiplicity $(\mathrm{s}=$ singlet, $\mathrm{d}=$ doublet, $\mathrm{t}=$ triplet, $\mathrm{q}=$ quartet, $\mathrm{br}=$ broad, $\mathrm{m}=$ multiplet), coupling constants $(\mathrm{Hz})$, integration and assignment. ${ }^{13} \mathrm{C}$ NMR spectra were recorded on a Varian Gemini $2000(100 \mathrm{MHz})$ spectrometer with complete proton decoupling. Chemical shifts are reported in ppm from tetramethylsilane with the solvent as the internal reference $\left(\mathrm{CDCl}_{3}: \delta\right.$ 77.16, $\left(\mathrm{CD}_{3}\right)_{2} \mathrm{SO}: \delta$ 39.52). Enantiomer ratios were determined by chiral HPLC (Chiral Technologies chiralpak OD column (4.6 mm x 250 $\mathrm{mm}$ )) or by chiral GLC analysis (Alltech Associates Chiraldex GTA or BPH, or Supelco Betadex or Alphadex 120 column $(30 \mathrm{~m} \times 0.25 \mathrm{~mm})$ ) in comparison with authentic racemic materials. Elemental analyses were performed at Robertson Microlit Laboratories (Madison, NJ). High-resolution mass spectrometry was performed at the Mass Spectrometry Facility, Boston College. Optical rotation values were recorded on a Rudolph Research Analytical Autopol IV polarimeter.

Materials: Solvents were purged with argon and then purified under a positive pressure of dry argon by a modified Innovative Technologies purification system: $\mathrm{Et}_{2} \mathrm{O}, \mathrm{CH}_{2} \mathrm{Cl}_{2}$, and THF were passed through activated alumina columns; benzene, toluene, and pentane ${ }^{1}$ were passed successively through activated $\mathrm{Cu}$ and alumina columns. Unless otherwise stated, commercially available reagents were used as received. Mo complexes $\mathbf{1 a}, \mathbf{1 b}{ }^{2}$ 1c, $3 \mathbf{a}, 3 \mathbf{3 b}, \mathbf{3 c},{ }^{3} \mathbf{2 a}, \mathbf{2 b},{ }^{4} \mathbf{2 c},{ }^{5} 4,{ }^{6} \mathbf{5}^{7}$ and $\mathbf{3 5}^{8}$ were prepared according to published

(1) $n$-Pentane was stirred over concentrated $\mathrm{H}_{2} \mathrm{SO}_{4}$ for 5 days, washed with a saturated aqueous solution of $\mathrm{NaHCO}_{3}$ followed by water, dried over $\mathrm{MgSO}_{4}$, and filtered before use in the solvent purification system.

(2) Alexander, J. B.; La, D. S.; Cefalo, D. R.; Hoveyda, A. H.; Schrock, R. R. J. Am. Chem. Soc. 1998, 120, 4041-4042.

(3) Schrock, R. R.; Jamieson, J. Y.; Dolman, S. J.; Miller, S. A.; Bonitatebus, P. J., Jr.; Hoveyda, A. H., Organometallics, 2002, 21, 409-417. 
procedures. Mo complexes were handled under an inert atmosphere in a dry box. Olefin metathesis substrates were dried by repeated (three times) azeotropic distillation of water with benzene under high vacuum (except amines 30 and 32: distilled from metallic sodium under vacuum prior to use).

Silica gel column chromatography was driven with compressed $\mathrm{N}_{2}$ or by gravity elution. For alkyl amines, Dragendorff's reagent was used to visualize TLC spots. Chromatography solvent buffered with ammonia was prepared by washing the organic solvent components (usually a $\mathrm{CH}_{2} \mathrm{Cl}_{2}: \mathrm{MeOH}$ mixture) with $2 \%$ by volume concentrated $\mathrm{NH}_{4} \mathrm{OH}$. The resulting organic solution was separated from the aqueous phase and used directly as solvent for elution.

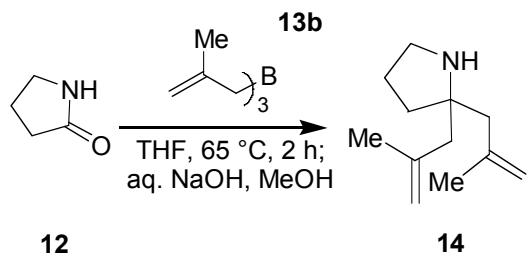

2,2-Bis-(2-methyl-allyl)-pyrrolidine 14. Amine 14 was prepared through a reductive bisalkylation according to a literature procedure with slight modification (see below). ${ }^{9}$ The trimethallyl borane reagent was prepared ( $85 \%$ yield, $44 \mathrm{mmol}$ ) and purified according to a procedure reported by Brown and coworkers for the synthesis of triallyl borane $\left(\mathrm{BF}_{3} \mathrm{OEt}_{2}\right.$, methallyl chloride, metallic $\left.\mathrm{Mg}, \mathrm{Et}_{2} \mathrm{O}\right){ }^{10}$ 2,5-dimethyl-hexa-1,5-diene, a byproduct formed during the preparation of trimethallyl borane, was removed through vacuum distillation $(\sim 2 \mathrm{~mm})$ of the mixture. Further distillation under high vacuum $(\sim 2$ $\mathrm{mm})$ with mild heating $\left(\sim 50{ }^{\circ} \mathrm{C}\right)$ delivered the desired trimethallyl borane in $80-85 \%$ yield. This compound is air and moisture sensitive and may be stored for short periods under an inert atmosphere.

To a cold $\left(0{ }^{\circ} \mathrm{C}\right)$ stirring solution of pyrrolidinone $(2.16 \mathrm{~mL}, 28.5 \mathrm{~mol}$, distilled before use) in $10 \mathrm{~mL}$ THF was added a solution of trimethallyl borane $(6.51 \mathrm{~g}, 37.0$ mmol in $3 \mathrm{~mL}$ THF) via cannula. THF ( $\sim 2 \mathrm{~mL})$ was used to complete the transfer. The reaction was heated at reflux for $1.5 \mathrm{~h}$ and then stirred for an additional $12 \mathrm{~h}$ at ambient temperature. At this time, $5 \mathrm{~mL}$ of $\mathrm{MeOH}$ was added. After $1 \mathrm{~h}, \sim 15 \mathrm{~mL}$ of an aqueous solution of $\mathrm{NaOH}(3 \mathrm{M})$ was added. Stirring was continued for an additional hour to ensure complete deboronation. The biphasic solution was then transferred to a separatory funnel with EtOAc. The aqueous layer was separated and washed $3 \mathrm{x}$ with $20 \mathrm{~mL}$ portions of EtOAc. The organic fractions were combined, dried over $\mathrm{K}_{2} \mathrm{CO}_{3}$, filtered and concentrated to give a clear oil (4.23 g, $23.6 \mathrm{mmol}, 83 \%)$. Analysis by ${ }^{1} \mathrm{H}$ NMR

(4) Zhu, S. S.; Cefalo, D. R.; La, D. S.; Jamieson, J. Y.; Davis, W. M.; Hoveyda, A. H.; Schrock R. R. J. Am. Chem. Soc. 1999, 121, 8251-8259.

(5) Cefalo, D. R.; Kiely, A. F.; Wuchrer, M.; Jamieson, J. Y.; Schrock, R. R.; Hoveyda, A. H. J. Am. Chem. Soc. 2001, 123, 3139-3140.

(6) Unpublished results of Dr. A. F. Kiely, Boston College, Chestnut Hill, MA.

(7) Tsang, W. C. P.; Jernelius, J. A.; Cortez, G. A.; Weatherhead, G. S.; Schrock, R. R.; Hoveyda, A. H. J. Am. Chem. Soc. 2003, 125, 2591-2596.

(8) Murdzek, J. S.; Schrock, R. R. Organometallics 1987, 6, 1373-1374.

(9) Bubnov, Y. N.; Pastukhov, F. V.; Yampolsky, I. V.; Ignatenko, A. V. Eur. J. Org. Chem. 2000, 15031505.

(10) Brown. H. C.; Racherla, U. S. J. Org. Chem. 1986, 51, 427-432. 
indicated that the product was $>98 \%$ pure. All spectral data were in accordance with that previously reported by Bubnov. ${ }^{9}$
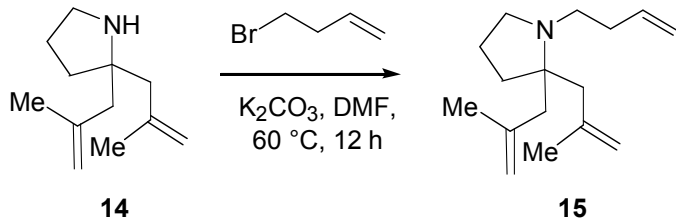

15. Amine 14 was combined with 1 -bromo,3-butene ( 2 equiv) and $\mathrm{K}_{2} \mathrm{CO}_{3}$ (1 equiv) in $\operatorname{DMF}\left(0.1 \mathrm{M}\right.$ with respect to 14). The solution was then heated to $60{ }^{\circ} \mathrm{C}$ for $12 \mathrm{~h}$. The mixture was then transferred to a separatory funnel and diluted with $\mathrm{Et}_{2} \mathrm{O}$ and $\mathrm{H}_{2} \mathrm{O}$. The aqueous layer was washed three times with $\mathrm{Et}_{2} \mathrm{O}$. The combined organic fractions were dried with $\mathrm{MgSO}_{4}$, filtered and concentrated to give a yellow oil. Analysis by ${ }^{1} \mathrm{~N} \mathrm{NMR}$ indicated $30-40 \%$ conversion to $\mathbf{1 5}$ for several reactions ranging from $0.07 \mathrm{mmol}$ to 3.00 mmol in scale. Purification of $\mathbf{1 5}$ was accomplished with silica gel chromatography (20:1 hexane: $\mathrm{Et}_{2} \mathrm{O}$ washed with $1 \% \mathrm{v} / \mathrm{v}$ concentrated $\mathrm{NH}_{4} \mathrm{OH}$ as the eluent). The substrate was obtained as a clear oil after Kugelrohr distillation over metallic Na. IR (neat): 3081 (m), 2956 (s), 2800 (m), 1650 (m), 1462 (m), 1381 (w), 900 (s) $\mathrm{cm}^{-1} ;{ }^{1} \mathrm{H}$ NMR (400 MHz, $\mathrm{CDCl}_{3}$ ): $\delta 5.84$ (ddt, $J=16.8,9.9,6.6 \mathrm{~Hz}, 1 \mathrm{H}, \mathrm{RCH}=\mathrm{CH}_{2}$ ), 5.05 (ddd, $J=17.2,3.7,1.5$ $\left.\mathrm{Hz}, 1 \mathrm{H}, \mathrm{RCH}=\mathrm{CH}_{\mathrm{A}} \mathrm{H}_{\mathrm{B}}\right), 4.98\left(\mathrm{~d}(\mathrm{br}), J=10.3 \mathrm{~Hz}, 1 \mathrm{H}, \mathrm{RCH}=\mathrm{CH}_{\mathrm{A}} \mathbf{H}_{\mathbf{B}}\right), 4.84(\mathrm{dd}, J=2.9$, $\left.1.5 \mathrm{~Hz}, 2 \mathrm{H}, \mathrm{R}(\mathrm{Me}) \mathrm{C}=\mathrm{CH}_{\mathrm{A}} \mathrm{H}_{\mathrm{B}}\right), 4.65\left(\mathrm{~s}(\mathrm{br}), 2 \mathrm{H}, \mathrm{R}(\mathrm{Me}) \mathrm{C}=\mathrm{CH}_{\mathrm{A}} \mathbf{H}_{\mathrm{B}}\right), 2.76(\mathrm{t}, J=7.0,2 \mathrm{H}$, $\left.\mathrm{NCH}_{2}\right), 2.53\left(\mathrm{t}, J=7.7 \mathrm{~Hz}, 2 \mathrm{H}, \mathrm{NCH}_{2}\right), 2.19$ (dd, $J=15.7,7.0 \mathrm{~Hz}, 2 \mathrm{H}, \mathrm{H}_{2} \mathrm{C}=\mathrm{CHCH}$ ), $2.11\left(\mathrm{ABq}, J=13.6 \mathrm{~Hz}, 4 \mathrm{H}, \mathrm{H}_{2} \mathrm{C}=\mathrm{CMeCH}_{2}\right), 1.80\left(\mathrm{~s}, 6 \mathrm{H}, \mathrm{CH}_{3}\right), 1.76-1.65(\mathrm{~m}, 4 \mathrm{H}$, $\left.\mathrm{NCR}_{2} \mathrm{CH}_{\mathbf{2}} \mathrm{CH}_{2}\right) ;{ }^{13} \mathrm{C}$ NMR $\left(100 \mathrm{MHz}, \mathrm{CDCl}_{3}\right): \delta 144.7,138.1,115.8,114.9,66.7,50.8$, 48.3, 42.2, 34.7, 32.2, 25.8, 21.7; HRMS Calcd for $\mathrm{C}_{16} \mathrm{H}_{27} \mathrm{~N}(\mathrm{M}+\mathrm{H})$ : 234.2222, Found: 234.2219 .

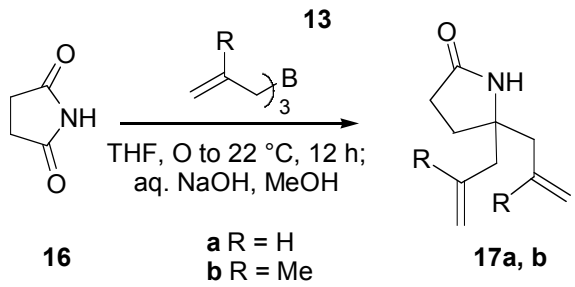

5,5-Bis-allyl-pyrrolidin-2-one 17 a and 5,5-bis-(2-methyl-allyl)-pyrrolidin-2-one 17 b. The procedure for the synthesis of $\mathbf{1 4}$ (see above) was adapted for the preparation of $17 \mathbf{a}, \mathbf{b}$.

17b. Succinimide (recrystallized from $\mathrm{C}_{6} \mathrm{H}_{6}, 4.62 \mathrm{~g}, 46.6 \mathrm{mmol}$ ) was ground to a fine powder and diluted with $93 \mathrm{~mL}$ THF. After all solids had dissolved, the mixture was cooled to $0{ }^{\circ} \mathrm{C}$ in an ice bath. Trimethallyl borane $(8.20 \mathrm{~g}, 46.6 \mathrm{mmol}, 1$ equiv.) was added over $\sim 20 \mathrm{~min}$ via cannula to the stirring solution, causing it to change from colorless to yellow. The reaction was then stirred for $12 \mathrm{~h}$ at ambient temperature. $\mathrm{MeOH}, 5 \mathrm{~mL}$, was added and stirring was continued for $0.5 \mathrm{~h}$. Subsequently, $\sim 5 \mathrm{~mL}$ of a $3 \mathrm{M}$ aqueous solution of $\mathrm{NaOH}$ was added and the resulting orange mixture was stirred for an additional hour. At this time, the solution was diluted with $\sim 30 \mathrm{~mL} \mathrm{Et}_{2} \mathrm{O}$ and 120 $\mathrm{mL}$ of a saturated aqueous solution of $\mathrm{NaCl}$. The organic layer was separated and washed with an additional $\sim 120 \mathrm{~mL}$ of a saturated aqueous solution of $\mathrm{NaCl}$. The remaining 
material was extracted from the combined aqueous layers with two $100 \mathrm{~mL}$ portions of $\mathrm{CH}_{2} \mathrm{Cl}_{2}$. The combined organic fractions were dried over $\mathrm{MgSO}_{4}$, filtered, and concentrated in vacuo to afford an orange foam. This material was absorbed onto silica gel and loaded onto of a silica column $\left(\sim 7 \mathrm{x} 4\right.$ inches). The product was eluted with $\mathrm{Et}_{2} \mathrm{O}$ washed with $1 \% \mathrm{v} / \mathrm{v}$ concentrated $\mathrm{NH}_{4} \mathrm{OH}$. A clean sample (by TLC analysis) of $\mathbf{1 7 b}$ $(3.00 \mathrm{~g})$ was obtained which could be recrystallized from hot $\mathrm{Et}_{2} \mathrm{O}$ to give off-white needles. A second fraction ( $3.15 \mathrm{~g}$ ) of $\mathbf{1 7} \mathbf{b}\left(\sim 90 \%\right.$ clean as judged by ${ }^{1} \mathrm{H}$ NMR analysis) was resubjected to the flash conditions for further purification (anisaldehyde for TLC visualization). Combined yield of $\mathbf{1 7 b}$ : $5.85 \mathrm{~g}(30.3 \mathrm{mmol}, 65 \%)$. IR (neat): 3211 (w), $3080(\mathrm{w}), 2973$ (w), 2936 (w), 1696 (s), 1446 (w), 1383 (w), 901 (m) $\mathrm{cm}^{-1}$; ${ }^{1} \mathrm{H}$ NMR (400 MHz, $\left.\mathrm{CDCl}_{3}\right): \delta 5.70(\mathrm{~s}(\mathrm{br}), 1 \mathrm{H}, \mathrm{NH}), 4.96\left(\mathrm{t}(\mathrm{br}), J=1.5 \mathrm{~Hz}, 2 \mathrm{H}, \mathrm{R}_{2} \mathrm{C}=\mathrm{CH}_{\mathrm{A}} \mathrm{H}_{\mathrm{B}}\right.$ ), $4.79\left(\mathrm{~s}(\mathrm{br}), 2 \mathrm{H}, \mathrm{R}_{2} \mathrm{C}=\mathrm{CH}_{\mathrm{A}} \mathbf{H}_{\mathrm{B}}\right), 2.34\left(\mathrm{t}, J=7.7 \mathrm{~Hz}, 2 \mathrm{H}, \mathrm{OCCH}_{2}\right), 2.27(\mathrm{ABd}, J=13.9 \mathrm{~Hz}$, $\left.4 \mathrm{H}, \mathrm{H}_{2} \mathrm{C}=\mathrm{C}(\mathrm{Me}) \mathrm{CH}_{2} \mathrm{R}\right), 2.08\left(\mathrm{t}, J=8.1 \mathrm{~Hz}, 2 \mathrm{H}, \mathrm{OCCH}_{2} \mathrm{CH}_{2} \mathrm{R}\right), 1.80\left(\mathrm{~s}, 6 \mathrm{H}, \mathrm{CH}_{3}\right) ;{ }^{13} \mathrm{C}$ NMR $\left(100 \mathrm{MHz}, \mathrm{CDCl}_{3}\right) ; \delta 177.9,141.5,115.9,62.3,48.6,31.1,29.6,24.5$; HRMS Calcd for $\mathrm{C}_{12} \mathrm{H}_{19} \mathrm{NO}(\mathrm{M}+\mathrm{H}):$ 194.1545, Found: 194.1543 .

17a. An analogous procedure to that for the synthesis of $\mathbf{1 7 b}$ was followed with triallyl borane as the alkylating agent. ${ }^{10}$ The desired product was obtained in $39 \%$ yield $(18 \mathrm{mmol})$ after purification by silica gel chromatography $\left(1: 1 \mathrm{Et}_{2} \mathrm{O}: \mathrm{EtOAc}\right.$; CAM stain for TLC visualization of product, anisaldehyde stain for TLC visualization of impurities). IR (neat): 3226 (s), 3087 (s), 2917 (s), 1715 (s), 1438 (m), 1313 (m), 1017 (m) cm ${ }^{-1} ;{ }^{1} \mathrm{H}$ NMR (400 MHz, $\mathrm{CDCl}_{3}$ ): $\delta 5.93(\mathrm{~s}(\mathrm{br}), 1 \mathrm{H}, \mathrm{NH}), 5.78$ (ddt, $J=17.1,10.3,7.8 \mathrm{~Hz}, 2 \mathrm{H}$, $\left.\mathrm{RCH}=\mathrm{CH}_{2}\right), 5.18\left(\mathrm{~d}(\mathrm{br}), J=7.8 \mathrm{~Hz}, 2 \mathrm{H}, \mathrm{RCH}=\mathrm{CH}_{\mathrm{A}} \mathrm{H}_{\mathrm{B}}\right), 5.15(\mathrm{~d}(\mathrm{br}), J=17.1 \mathrm{~Hz}, 2 \mathrm{H}$, $\left.\mathrm{RCH}=\mathrm{CH}_{\mathrm{A}} \mathbf{H}_{\mathbf{B}}\right), 2.36\left(\mathrm{t}, J=7.8 \mathrm{~Hz}, 2 \mathrm{H}, \mathrm{OCCH}_{2}\right), 2.31(\mathrm{dd}, J=13.7,6.3 \mathrm{~Hz}, 2 \mathrm{H}$, $\left.\mathrm{RCH}_{\mathrm{A}} \mathrm{H}_{\mathrm{B}} \mathrm{CH}=\mathrm{CH}_{2}\right), 2.23\left(\mathrm{dd}, J=14.2,8.3 \mathrm{~Hz}, 2 \mathrm{H}, \mathrm{RCH}_{\mathrm{A}} \mathbf{H}_{\mathrm{B}} \mathrm{CH}=\mathrm{CH}_{2}\right), 1.96(\mathrm{t}, J=7.8$ $\left.\mathrm{Hz}, 2 \mathrm{H}, \mathrm{OCCH}_{2} \mathrm{CH}_{2} \mathrm{R}\right) ;{ }^{13} \mathrm{C} \mathrm{NMR}\left(100 \mathrm{MHz}, \mathrm{CDCl}_{3}\right) ; \delta 178.0,132.7,119.5,61.5,44.6$, 30.7, 30.1; HRMS Calcd for $\mathrm{C}_{10} \mathrm{H}_{15} \mathrm{NO}(\mathrm{M}+\mathrm{H})$ : 166.1232, Found: 166.1226.

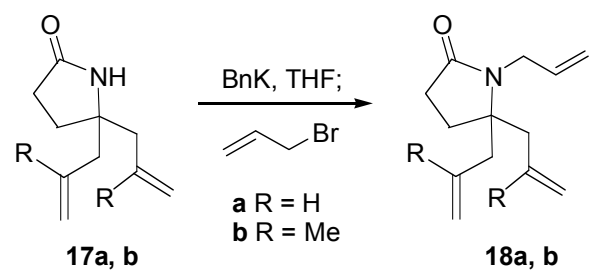

18a and 18b. The procedure for the $N$-alkylation of $\mathbf{1 7 b}$ (for the preparation of 19) was followed (see below) with allyl bromide as the electrophile. In both cases, ${ }^{1} \mathrm{H}$ NMR analysis of the unpurified reaction mixture indicated $>98 \%$ conversion to the desired product.

18b. Silica gel chromatography ( $4: 1 \mathrm{Et}_{2} \mathrm{O}$ :pentane) gave the desired triene $\mathbf{1 8 b}$ (5 mmol) in 95\% yield as an off-white solid. IR (neat): 3087 (w), 2974 (m), 1703 (s), 1652 (w), $1457(\mathrm{w}), 1407(\mathrm{~m}), 910(\mathrm{~m}) \mathrm{cm}^{-1} ;{ }^{1} \mathrm{H} \mathrm{NMR}$ (400 MHz, $\mathrm{CDCl}_{3}$ ): $\delta 5.87$ (ddt, $J=$ $\left.17.2,10.3,5.9 \mathrm{~Hz}, 1 \mathrm{H}, \mathrm{RCH}=\mathrm{CH}_{2}\right), 5.19\left(\mathrm{ddt}, J=17.2,1.5,1.5 \mathrm{~Hz}, 1 \mathrm{H}, \mathrm{RCH}=\mathrm{CH}_{\mathrm{A}} \mathrm{H}_{\mathrm{B}}\right)$, $5.12\left(\mathrm{ddt}, J=10.3,1.5,1.5 \mathrm{~Hz}, 1 \mathrm{H}, \mathrm{RCH}=\mathrm{CH}_{\mathrm{A}} \mathbf{H}_{\mathbf{B}}\right), 4.90(\mathrm{tt}, J=1.5,1.5 \mathrm{~Hz}, 2 \mathrm{H}$, $\left.\mathrm{R}(\mathrm{Me}) \mathrm{C}=\mathrm{CH}_{\mathrm{A}} \mathrm{H}_{\mathrm{B}}\right), 4.75\left(\mathrm{~s}(\mathrm{br}), 2 \mathrm{H}, \mathrm{R}(\mathrm{Me}) \mathrm{C}=\mathrm{CH}_{\mathrm{A}} \mathbf{H}_{\mathbf{B}}\right), 3.83(\mathrm{dt}, J=5.5,1.5 \mathrm{~Hz}, 2 \mathrm{H}$, $\left.\mathrm{NCH}_{2}\right), 2.35-2.23(\mathrm{~m}, 6 \mathrm{H}), 2.11(\mathrm{t}, J=8.4 \mathrm{~Hz}, 2 \mathrm{H}), 1.75\left(\mathrm{~s}, 6 \mathrm{H}, \mathrm{CH}_{3}\right) ;{ }^{13} \mathrm{C} \mathrm{NMR}(100$ 
$\left.\mathrm{MHz}, \quad \mathrm{CDCl}_{3}\right): \quad \delta 175.3,141.2,134.7,116.7,116.3,66.8,46.8,43.1,30.4,29.3,24.7$; HRMS Calcd for $\mathrm{C}_{15} \mathrm{H}_{23} \mathrm{NO}(\mathrm{M}+\mathrm{Na}): 256.1677$, Found: 256.1681 .

18a. Silica gel chromatography $\left(2: 1 \mathrm{Et}_{2} \mathrm{O}\right.$ :hexane) gave the desired triene $\mathbf{1 8 b}(1.5$ $\mathrm{mmol}$ ) in 85\% yield as an off-white solid. IR (neat): 3081 (w), 2981 (w), 2925 (w) 1693 (s), 1406 (m), 1000 (w), 919 (m) cm ${ }^{-1} ;{ }^{1} \mathrm{H}$ NMR (400 MHz, $\left.\mathrm{CDCl}_{3}\right): \delta 5.89$ (ddt, $J=17.2$, $\left.10.3,6.2 \mathrm{~Hz}, 1 \mathrm{H}, \mathrm{NCH}_{2} \mathrm{CH}=\mathrm{CH}_{2}\right), 5.73-5.63\left(\mathrm{~m}, 2 \mathrm{H}, \mathrm{NCR}\left(\mathrm{CH}_{2} \mathrm{CH}=\mathrm{CH}_{2}\right)_{2}\right), 5.21$ (ddt, $J$ $\left.=17.2,1.5,1.5 \mathrm{~Hz}, 1 \mathrm{H}, \mathrm{CH}=\mathrm{CH}_{2}\right), 5.15-5.10\left(\mathrm{~m}, 5 \mathrm{H}, \mathrm{CH}=\mathrm{CH}_{2}\right), 3.84(\mathrm{~d}(\mathrm{br}), J=6.2 \mathrm{~Hz}$, $\left.2 \mathrm{H}, \mathrm{NCH}_{2} \mathrm{R}\right), 2.38\left(\mathrm{ddt}, J=14.3,6.2,1.5 \mathrm{~Hz}, 2 \mathrm{H}, \mathrm{R}\left(\mathrm{CH}_{\mathrm{A}} \mathrm{H}_{\mathrm{B}} \mathrm{CH}=\mathrm{CH}_{2}\right)_{2}\right), 2.32(\mathrm{t}, J=8.4$ $\mathrm{Hz}, 2 \mathrm{H}), 2.24$ (ddt, $\left.J=13.9,8.1,1.0 \mathrm{~Hz}, 2 \mathrm{H}, \mathrm{R}\left(\mathrm{CH}_{\mathrm{A}} \mathbf{H}_{\mathrm{B}} \mathrm{CH}=\mathrm{CH}_{2}\right)_{2}\right), 1.91$ (t, $J=8.4 \mathrm{~Hz}$, $\left.{ }_{2 \mathrm{H}}\right) ; \quad{ }^{13} \mathrm{C} \quad \mathrm{NMR} \quad\left(100 \quad \mathrm{MHz}, \quad \mathrm{CDCl}_{3}\right)$ : $\delta$ 175.4, 134.6, 132.7, 119.6, 117.1, 65.9, 43.7, 42.8, 29.9, 27.5; HRMS Calcd for $\mathrm{C}_{13} \mathrm{H}_{19} \mathrm{NO}(\mathrm{M}+\mathrm{Na}): 228.1364$, Found: 228.1363.
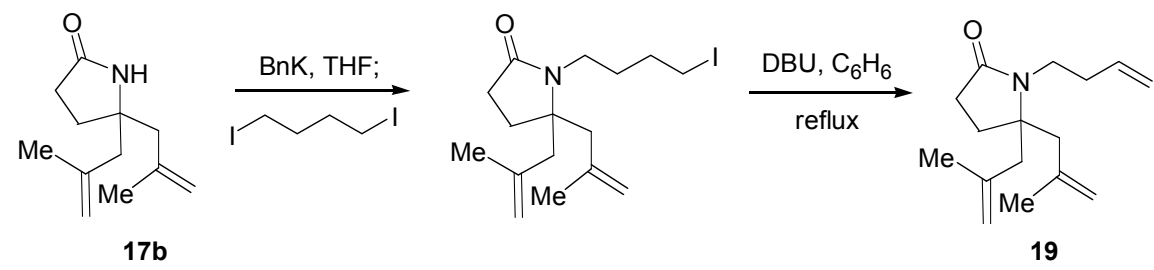

19. In a $\mathrm{N}_{2}$ filled glovebox, $\mathbf{1 7 b}(659 \mathrm{mg}, 3.41 \mathrm{mmol})$ was dissolved in $17 \mathrm{~mL}$ THF and cooled to $-30{ }^{\circ} \mathrm{C}$. Benzyl potassium ${ }^{11}(444 \mathrm{mg}, 3.41 \mathrm{mmol}$ ) was added in portions. (If necessary, additional Benzyl potassium was added just until the orange color persists.) The mixture was sealed, removed from the glove box and cooled to $0{ }^{\circ} \mathrm{C}$. The electrophile, 1,5 diiodo pentane (Aldrich, $1.32 \mathrm{~mL}, 10.2 \mathrm{mmol}$ ), was added drowise via syringe. The resulting white mixture was stirred for $12 \mathrm{~h}$, at which time $\sim 40 \mathrm{~mL}$ of a saturated aqueous solution of $\mathrm{NaCl}$ was added. The organic layer was separated and washed with two additional portions $(\sim 20 \mathrm{~mL})$ of a saturated aqueous solution of $\mathrm{NaCl}$. The combined aqueous layers were then washed with two $50 \mathrm{~mL}$ portions of $\mathrm{CH}_{2} \mathrm{Cl}_{2}$. The organic fractions were combined, dried over $\mathrm{MgSO}_{4}$, and concentrated in vacuo. ${ }^{1} \mathrm{H}$ NMR analysis of the crude reaction mixture indicated $\sim 70 \%$ conversion to the desired product. Silica gel chromatography (4:1 pentane: $\left.\mathrm{Et}_{2} \mathrm{O}\right)$ of the mixture gave $520 \mathrm{mg}$ of 1(4-iodo-butyl)-5,5-bis-(2-methyl-allyl)-pyrrolidin-2-one ( $\sim 40 \%$ yield) combined with $\sim 5 \%$ 19. A solution of this material $(346 \mathrm{mg})$ in $9 \mathrm{~mL} \mathrm{C}_{6} \mathrm{H}_{6}$ was heated to reflux in the presence of $0.8 \mathrm{~mL}$ DBU ( $\sim 6$ equiv, distilled from $\mathrm{CaH}_{2}$ ) for $1 \mathrm{~h}$. At this time, white solids had precipitated from the reaction mixture and TLC analysis indicated complete consumption of starting material. Pentane $(\sim 20 \mathrm{~mL})$ was added, causing further precipitation of the ammonium salts, and the resulting solution was filtered. Concentration of the filtrate gave an oil that was purified by silica gel chromatography (4:1 $\mathrm{Et}_{2} \mathrm{O}$ :pentane) to give $59.4 \mathrm{mg}$ of $19(0.240 \mathrm{mmol}, 26 \%$ yield). IR (neat): $3074(\mathrm{w})$, $2974(\mathrm{~m}), 2930$ (m), 1697 (s), 1463 (m), 1406 (m), 1375 (w), 904 (m) $\mathrm{cm}^{-1}$; ${ }^{1} \mathrm{H}$ NMR $\left(400 \mathrm{MHz}, \mathrm{CDCl}_{3}\right.$ ): $\delta 5.82$ (ddt, $J=17.2,10.3,7.0 \mathrm{~Hz}, 1 \mathrm{H}, \mathrm{RCH}=\mathrm{CH}_{2}$ ), 5.09 (ddd, $J=$ $\left.17.2,3.3,1.8 \mathrm{~Hz}, 1 \mathrm{H}, \mathrm{RCH}=\mathrm{CH}_{\mathrm{A}} \mathrm{H}_{\mathrm{B}}\right), 5.04\left(\mathrm{~d}(\mathrm{br}), J=10.3 \mathrm{~Hz}, 1 \mathrm{H}, \mathrm{RCH}=\mathrm{CH}_{\mathrm{A}} \mathbf{H}_{\mathbf{B}}\right), 4.91$ (t(br), $\left.J=1.5 \mathrm{~Hz}, 2 \mathrm{H}, \mathrm{R}(\mathrm{Me}) \mathrm{C}=\mathrm{CH}_{\mathrm{A}} \mathrm{H}_{\mathrm{B}}\right), 4.76\left(\mathrm{~s}(\mathrm{br}), 2 \mathrm{H}, \mathrm{R}(\mathrm{Me}) \mathrm{C}=\mathrm{CH}_{\mathrm{A}} \mathbf{H}_{\mathbf{B}}\right), 3.19-3.15$

(11) Lochmann, L.; Trekoval, J. J. Organomet. Chem. 1987, 326, 1. 
(m, 2H, NCH $), 2.39-2.28(\mathrm{~m}, 6 \mathrm{H}), 2.24(\mathrm{t}, J=13.9 \mathrm{~Hz}, 2 \mathrm{H}), 2.09(\mathrm{t}, J=8.0 \mathrm{~Hz}, 2 \mathrm{H})$, $1.76\left(\mathrm{~s}, 6 \mathrm{H}, \mathrm{CH}_{3}\right) ;{ }^{13} \mathrm{C} \mathrm{NMR}\left(100 \mathrm{MHz}, \mathrm{CDCl}_{3}\right): \delta 175.5,141.2,135.6,116.7,116.4$, 66.6, 46.9, 40.4, 33.9, 30.5, 29.4, 24.7; HRMS Calcd for $\mathrm{C}_{16} \mathrm{H}_{25} \mathrm{NO}(\mathrm{M}+\mathrm{Na})$ : 270.1834, Found: 270.1835 .

General procedure for Mo-catalyzed asymmetric ring-closing metathesis (ARCM) under inert $\left(\mathbf{N}_{2}\right)$ atmosphere: (Reactions performed entirely in an $\mathrm{N}_{2}$ dry box). A one dram vial was charged with a solution of $\mathbf{5 1}(40 \mathrm{mg}, 0.12 \mathrm{mmol})$ in $1.2 \mathrm{~mL}^{\circ} \mathrm{C}_{6} \mathrm{H}_{6}$. Mo catalyst $1 \mathbf{b}(4.4 \mathrm{mg}, 5.9 \mu \mathrm{mol}, 5 \mathrm{~mol} \%)$ was added in one portion. The vial was sealed with a cap and the reaction was stirred at ambient temperature for $24 \mathrm{~h}$. At this time, the vial was removed from the dry box, $\sim 1 \mathrm{~mL}$ of wet (nondistilled) $\mathrm{Et}_{2} \mathrm{O}$ was added to quench the catalyst and the resulting solution was concentrated in vacuo. ${ }^{1} \mathrm{H}$ NMR analysis of the unpurified reaction mixture indicated $\sim 95 \%$ conversion to the desired product (52). Silica gel chromatography ( $3: 1$ hexanes: $\left.\mathrm{Et}_{2} \mathrm{O}\right)$ was used to purify $\mathbf{5 2}$ in $\mathbf{9 4 \%}$ yield (35 mg, $0.11 \mathrm{mmol})$.

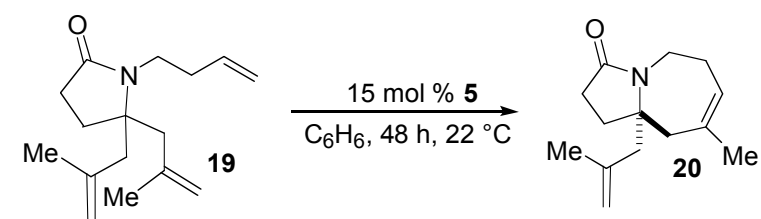

20. General procedure for ARCM was followed. Silica gel chromatography with $100 \%$ $\mathrm{Et}_{2} \mathrm{O}$ was used for purification (84\% yield, $0.083 \mathrm{mmol}$ ). IR (neat): $2967(\mathrm{~m}), 2924(\mathrm{~m})$, 1701 (s), $1438(\mathrm{~m}), 1413(\mathrm{~m}) \mathrm{cm}^{-1} ;{ }^{1} \mathrm{H}$ NMR $\left(400 \mathrm{MHz}, \mathrm{CDCl}_{3}\right): \delta 5.62(\mathrm{dd}(\mathrm{br}), J=5.1$, $\left.5.1 \mathrm{~Hz}, 1 \mathrm{H}, \mathrm{R}_{2} \mathrm{C}=\mathrm{CHR}\right), 4.89\left(\mathrm{dd}, J=1.8,1.8 \mathrm{~Hz}, 1 \mathrm{H}, \mathrm{R}_{2} \mathrm{C}=\mathrm{CH}_{\mathbf{A}} \mathrm{H}_{\mathrm{B}}\right), 4.73(\mathrm{~s}(\mathrm{br}), 1 \mathrm{H}$, $\mathrm{R}_{2} \mathrm{C}=\mathrm{CH}_{\mathrm{A}} \mathbf{H}_{\mathbf{B}}$ ), 4.05 (ddd, $J=13.6,4.0,4.0 \mathrm{~Hz}, 1 \mathrm{H}, \mathrm{NCH}_{\mathrm{A}} \mathrm{H}_{\mathrm{B}} \mathrm{R}$ ), 2.85 (ddd, $J=14.3$, $\left.12.1,2.9 \mathrm{~Hz}, 1 \mathrm{H}, \mathrm{NCH}_{\mathrm{A}} \mathbf{H}_{\mathrm{B}} \mathrm{R}\right), 2.61(\mathrm{~d}, J=14.3 \mathrm{~Hz}, 1 \mathrm{H}), 2.44(\mathrm{~d}, J=14.6 \mathrm{~Hz}, 1 \mathrm{H}), 2.41-$ $2.09(\mathrm{~m}, 6 \mathrm{H}), 2.04(\mathrm{~d}, J=13.9 \mathrm{~Hz}, 1 \mathrm{H}), 1.76\left(\mathrm{~s}, 3 \mathrm{H}, \mathrm{CH}_{3}\right), 1.75-1.72(\mathrm{~m}, 1 \mathrm{H}), 1.71(\mathrm{~s}, 3 \mathrm{H}$, $\left.\mathrm{CH}_{3}\right) ;{ }^{13} \mathrm{C}$ NMR $\left(100 \mathrm{MHz}, \mathrm{CDCl}_{3}\right): \delta 174.5,141.8,135.2,125.4,115.9,64.6,47.0,42.5$, 37.7, 30.4, 30.1, 28.4, 27.3, 24.2; HRMS Calcd for $\mathrm{C}_{14} \mathrm{H}_{21} \mathrm{NO}(\mathrm{M}+\mathrm{Na})$ : 242.1521, Found: $242.1517 ;[\alpha]_{\mathrm{D}}+14.9\left(c=0.5, \mathrm{CHCl}_{3}\right)$.

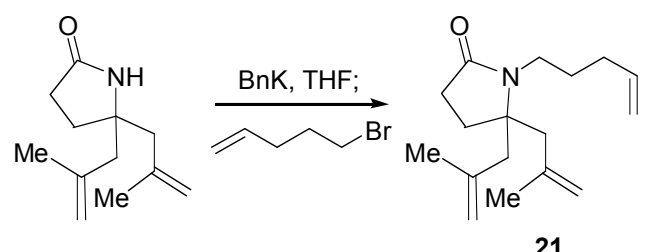

21. The procedure for the $N$-alkylation of $\mathbf{1 7 b}$ (for the preparation of 19) was followed (see above) with 5-bromo-1-pentene as the electrophile. ${ }^{1} \mathrm{H}$ NMR analysis of the unpurified reaction mixture indicated $\sim 60 \%$ conversion to the desired product. Silica gel chromatography (2:1 to $4: 1 \mathrm{Et}_{2} \mathrm{O}$ :pentane) gave the desired triene 21 (4 mmol) in 57\% yield as an off-white solid. IR (neat): 3087 (w), 2943 (m), $1690(\mathrm{~s}), 1444$ (m), 1407 (m), $1306(\mathrm{w}), 897(\mathrm{~m}) \mathrm{cm}^{-1} ;{ }^{1} \mathrm{H}$ NMR (400 MHz, $\mathrm{CDCl}_{3}$ ): $\delta 5.82$ (ddt, $J=16.8,10.3,6.6 \mathrm{~Hz}$, $1 \mathrm{H}, \mathrm{RCH}=\mathrm{CH}_{2}$ ), 5.05 (ddt, $J=17.2,1.8,1.8 \mathrm{~Hz}, 1 \mathrm{H}, \mathrm{RCH}=\mathrm{CH}_{\mathbf{A}} \mathrm{H}_{\mathrm{B}}$ ), 4.99 (ddt), $J=10.3$, $\left.2.6,1.7 \mathrm{~Hz}, 1 \mathrm{H}, \mathrm{RCH}=\mathrm{CH}_{\mathrm{A}} \mathbf{H}_{\mathbf{B}}\right), 4.91\left(\mathrm{tt}, J=2.9,1.5 \mathrm{~Hz}, 2 \mathrm{H}, \mathrm{R}(\mathrm{Me}) \mathrm{C}=\mathrm{CH}_{\mathbf{A}} \mathrm{H}_{\mathrm{B}}\right), 4.76$ (s(br), 2H, R(Me)C $\left.=\mathrm{CH}_{\mathrm{A}} \mathbf{H}_{\mathbf{B}}\right), 3.13-3.08\left(\mathrm{~m}, 2 \mathrm{H}, \mathrm{NCH}_{2}\right), 2.33-2.21(\mathrm{~m}, 6 \mathrm{H}), 2.13-2.07$ 
(m, 4H), $1.76\left(\mathrm{~s}, 6 \mathrm{H}, \mathrm{CH}_{3}\right), 1.75-1.66(\mathrm{~m}, 2 \mathrm{H})$; HRMS Calcd for $\mathrm{C}_{17} \mathrm{H}_{27} \mathrm{NO}(\mathrm{M}+\mathrm{Na})$ : 284.1990, Found: 284.1990.

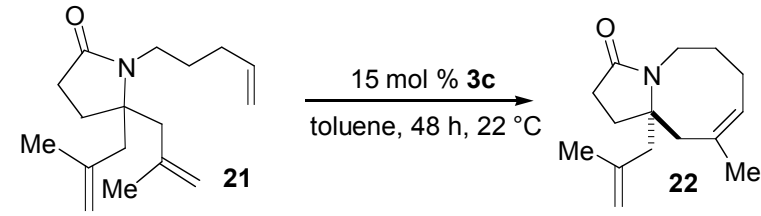

22. General procedure for ARCM was followed with some modification: toluene was used as a solvent and the vessel was loosely capped for the duration of the reaction. Silica gel chromatography with $100 \% \mathrm{Et}_{2} \mathrm{O}$ was used for purification $(90 \%$ yield, $0.088 \mathrm{mmol}$ ). IR (neat): 2936 (m), 2861 (m), 1697 (s), $1457(\mathrm{~m}), 1413(\mathrm{~m}), 13.76(\mathrm{~m}), 910(\mathrm{w}) \mathrm{cm}^{-1} ;{ }^{1} \mathrm{H}$ NMR (400 MHz, $\mathrm{CDCl}_{3}$ ): $\delta 5.56$ (dd(br), $J=8.4,8.4 \mathrm{~Hz}, 1 \mathrm{H}, \mathrm{R}_{2} \mathrm{C}=\mathrm{CHR}$ ), 4.91 (dd, $J=$ $\left.1.5,1.5 \mathrm{~Hz}, 1 \mathrm{H}, \mathrm{R}_{2} \mathrm{C}=\mathrm{CH}_{\mathrm{A}} \mathrm{H}_{\mathrm{B}}\right), 4.72\left(\mathrm{~s}(\mathrm{br}), 1 \mathrm{H}, \mathrm{R}_{2} \mathrm{C}=\mathrm{CH}_{\mathrm{A}} \mathbf{H}_{\mathbf{B}}\right), 3.63(\mathrm{~d}(\mathrm{br}), J=13.9 \mathrm{~Hz}$, $1 \mathrm{H}, \mathrm{NCH}_{\mathrm{A}} \mathrm{H}_{\mathrm{B}} \mathrm{R}$ ), 2.82 (ddd, $\left.J=13.9,13.9,3.7 \mathrm{~Hz}, 1 \mathrm{H}, \mathrm{NCH}_{\mathrm{A}} \mathbf{H}_{\mathrm{B}} \mathrm{R}\right), 2.48$ (d, $J=14.3 \mathrm{~Hz}$, $1 \mathrm{H}), 2.39-2.03(\mathrm{~m}, 8 \mathrm{H}), 1.97-1.89(\mathrm{~m}, 1 \mathrm{H}), 1.84\left(\mathrm{~s}, 3 \mathrm{H}, \mathrm{CH}_{3}\right), 1.85-1.79(\mathrm{~m}, 1 \mathrm{H}), 1.68(\mathrm{~s}$, $\left.3 \mathrm{H}, \mathrm{CH}_{3}\right), 1.49-1.43(\mathrm{~m}, 1 \mathrm{H}) ;{ }^{13} \mathrm{C} \mathrm{NMR}\left(100 \mathrm{MHz}, \mathrm{CDCl}_{3}\right): \delta 176.4,141.9,134.2,128.7$, 116.8, 68.0, 45.7 (br), 40.9, 30.4, 29.6, 27.7, 27.1, 26.3, 24.7; HRMS Calcd for $\mathrm{C}_{15} \mathrm{H}_{23} \mathrm{NO}(\mathrm{M}+\mathrm{Na}): 256.1677$, Found: $256.1669 ;[\alpha]_{\mathrm{D}}-27.2\left(c=1, \mathrm{CHCl}_{3}\right)$.
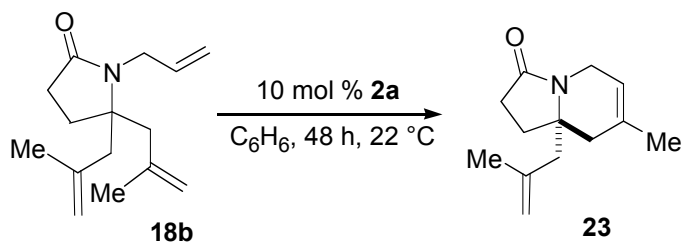

23. General procedure for ARCM was followed. Silica gel chromatography with 1:1 EtOAc: $\mathrm{Et}_{2} \mathrm{O}$ was used for purification (91\% yield, $\left.0.098 \mathrm{mmol}\right)$. IR (neat): $2974(\mathrm{~m})$, 2943 (m), 2861 (m), 1710 (s), 1457 (m), $1420(\mathrm{~m}), 904(\mathrm{w}) \mathrm{cm}^{-1} ;{ }^{1} \mathrm{H}$ NMR (400 MHz, $\left.\mathrm{CDCl}_{3}\right): \delta 5.38\left(\mathrm{~s}(\mathrm{br}), 1 \mathrm{H}, \mathrm{R}_{2} \mathrm{C}=\mathrm{CHR}\right), 4.90\left(\mathrm{~s}(\mathrm{br}), 1 \mathrm{H}, \mathrm{R}_{2} \mathrm{C}=\mathrm{CH}_{\mathrm{A}} \mathrm{H}_{\mathrm{B}}\right), 4.72(\mathrm{~s}(\mathrm{br}), 1 \mathrm{H}$, $\left.\mathrm{R}_{2} \mathrm{C}=\mathrm{CH}_{\mathrm{A}} \mathbf{H}_{\mathbf{B}}\right), 4.32\left(\mathrm{~d}(\mathrm{br}), J=18.3 \mathrm{~Hz}, 1 \mathrm{H}, \mathrm{NCH}_{\mathrm{A}} \mathrm{H}_{\mathrm{B}} \mathrm{R}\right), 3.43(\mathrm{~d}(\mathrm{br}), J=17.6 \mathrm{~Hz}, 1 \mathrm{H}$, $\left.\mathrm{NCH}_{\mathrm{A}} \mathbf{H}_{\mathrm{B}} \mathrm{R}\right), 2.48-2.22(\mathrm{~m}, 5 \mathrm{H}), 2.14-2.04(\mathrm{~m}, 2 \mathrm{H}), 1.82-1.75(\mathrm{~m}, 1 \mathrm{H}), 1.74\left(\mathrm{~s}, 3 \mathrm{H}, \mathrm{CH}_{3}\right)$, $1.69\left(\mathrm{~s}, 3 \mathrm{H}, \mathrm{CH}_{3}\right) ;{ }^{13} \mathrm{C}$ NMR $\left(100 \mathrm{MHz}, \mathrm{CDCl}_{3}\right): \delta 173.8,141.7,131.5,116.4,116.0$, 60.7, 43.7, 41.4, 38.6, 30.8, 30.0, 24.4, 23.6; HRMS Calcd for $\mathrm{C}_{13} \mathrm{H}_{19} \mathrm{NO}(\mathrm{M}+\mathrm{H})$ : 206.1545, Found: 206.1542. Anal. Calcd. for $\mathrm{C}_{13} \mathrm{H}_{19} \mathrm{NO}$ : C, 76.06; H, 9.33; Found: C, 76.16; $\mathrm{H}, 9.60 ;[\alpha]_{\mathrm{D}}+103.2\left(c=1, \mathrm{CHCl}_{3}\right)$.
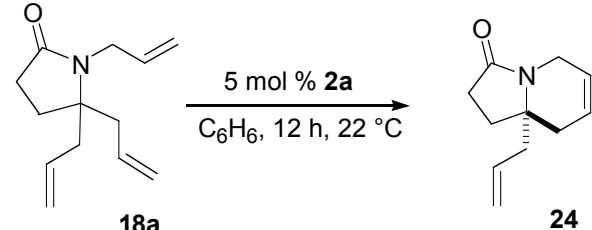

24. General procedure for ARCM was followed. Silica gel chromatography with 1:1 EtOAc: $\mathrm{Et}_{2} \mathrm{O}$ was used for purification $(92 \%$ yield, $0.122 \mathrm{mmol})$. IR (neat): $3075(\mathrm{w})$, 3037 (w), 2925 (m), 2843 (m), 1693 (s), 1419 (m), 1212 (w), 925 (w) cm ${ }^{-1}$; ${ }^{1} \mathrm{H}$ NMR $\left(400 \mathrm{MHz}, \mathrm{CDCl}_{3}\right): \delta 5.76-5.65\left(\mathrm{~m}, 3 \mathrm{H}, \mathrm{RCH}=\mathrm{CH}_{2}, \mathrm{RCH}=\mathrm{CHR}\right), 5.14(\mathrm{~s}(\mathrm{br}), 1 \mathrm{H}$, $\left.\mathrm{RCH}=\mathrm{CH}_{\mathbf{A}} \mathrm{H}_{\mathrm{B}}\right), 5.12-5.09\left(\mathrm{~m}, 1 \mathrm{H}, \mathrm{RCH}=\mathrm{CH}_{\mathrm{A}} \mathbf{H}_{\mathbf{B}}\right), 4.36(\mathrm{~d}(\mathrm{br}), J=19.0 \mathrm{~Hz}, 1 \mathrm{H}$, $\left.\mathrm{NCH}_{\mathbf{A}} \mathrm{H}_{\mathrm{B}} \mathrm{R}\right), 3.43\left(\mathrm{~d}(\mathrm{br}), J=18.7 \mathrm{~Hz}, 1 \mathrm{H}, \mathrm{NCH}_{\mathrm{A}} \mathbf{H}_{\mathbf{B}} \mathrm{R}\right), 2.47-2.31(\mathrm{~m}, 3 \mathrm{H}), 2.26-2.12(\mathrm{~m}$, $4 \mathrm{H}), 1.76$ (ddd, $J=12.8,9.9,6.6 \mathrm{~Hz}, 1 \mathrm{H}) ;{ }^{13} \mathrm{C}$ NMR $\left(100 \mathrm{MHz}, \mathrm{CDCl}_{3}\right): \delta 173.8,132.9$, 
123.8, 123.1, 119.5, 59.9, 40.8, 38.5, 36.3, 30.2, 29.8; HRMS Calcd for $\mathrm{C}_{11} \mathrm{H}_{15} \mathrm{NO}$ $(\mathrm{M}+\mathrm{Na})$ : 200.1051, Found: 200.1054; $[\alpha]_{\mathrm{D}}+57.7\left(c=1, \mathrm{CHCl}_{3}\right)$.
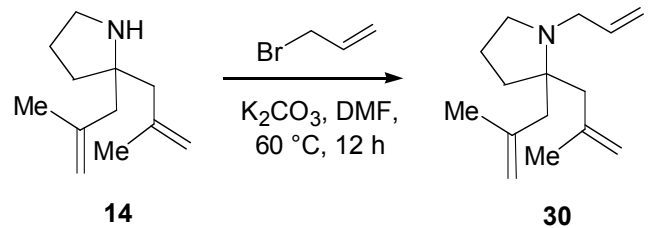

30. Amine 30 was prepared in $84 \%$ yield $(1.98 \mathrm{mmol})$ according to the procedure for the preparation of 15 with allyl bromide as the electrophile. IR (neat): $3075(\mathrm{~m}), 2968(\mathrm{~s})$, $2804(\mathrm{~m}), 1646$ (m), 1451 (m), $922(\mathrm{~m}), 878(\mathrm{~s}) \mathrm{cm}^{-1} ;{ }^{1} \mathrm{H}$ NMR (400 MHz, CDCl $)$ : $\delta 5.82$ (dddd, $\left.J=16.5,10.3,6.2,6.2 \mathrm{~Hz}, 1 \mathrm{H}, \mathrm{RCH}=\mathrm{CH}_{2}\right), 5.17$ (dd, $J=3.3,1.8 \mathrm{~Hz}, 1 \mathrm{H}$, $\left.\mathrm{RCH}=\mathrm{CH}_{\mathrm{A}} \mathrm{H}_{\mathrm{B}}\right), 5.13\left(\mathrm{dd}, J=3.3,1.5 \mathrm{~Hz}, 1 \mathrm{H}, \mathrm{RCH}=\mathrm{CH}_{\mathrm{A}} \mathbf{H}_{\mathrm{B}}\right), 5.03(\mathrm{~m}, 2 \mathrm{H}$, $\left.\mathrm{R}_{2} \mathrm{C}=\mathrm{CH}_{\mathrm{A}} \mathrm{H}_{\mathrm{B}}\right), 5.00\left(\mathrm{~m}, 2 \mathrm{H}, \mathrm{R}_{2} \mathrm{C}=\mathrm{CH}_{\mathrm{A}} \mathbf{H}_{\mathbf{B}}\right), 3.13(\mathrm{ddd}, J=6.2,1.5,1.5 \mathrm{~Hz}, 2 \mathrm{H}$, $\left.\mathrm{NCH}_{2} \mathrm{CH}=\mathrm{CH}_{2}\right), 2.72\left(\mathrm{dd}, J=7.0,7.0 \mathrm{~Hz}, 2 \mathrm{H}, \mathrm{NCH}_{2} \mathrm{CH}_{2} \mathrm{R}\right), 2.12(\mathrm{ABq}, J=13.6 \mathrm{~Hz}$, $\left.4 \mathrm{H}, \mathrm{R}\left(\mathrm{CH}_{2} \mathrm{C}\left(\mathrm{CH}_{3}\right)=\mathrm{CH}_{2}\right)_{2}\right), 1.81\left(\mathrm{~s}, 6 \mathrm{H}, \mathrm{CH}_{3}\right), 1.78-1.75(\mathrm{~m}, 2 \mathrm{H}), 1.71-1.63(\mathrm{~m}, 2 \mathrm{H}) ;{ }^{13} \mathrm{C}$ NMR (100 MHz, $\left.\mathrm{CDCl}_{3}\right): \delta 144.1,137.8,115.6,114.4,65.8,51.2,50.5,41.8,31.7,25.3$, 21.1; HRMS Calcd for $\mathrm{C}_{15} \mathrm{H}_{25} \mathrm{~N}(\mathrm{M}+\mathrm{H}): 220.2065$, Found: 220.2073 .

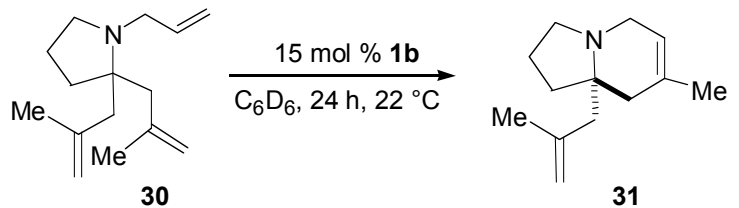

31. General procedure for ARCM was followed. Silica gel chromatography with $15: 1$ $\mathrm{CH}_{2} \mathrm{Cl}_{2}: \mathrm{MeOH}$ washed with $2 \% \mathrm{v} / \mathrm{v}$ concentrated $\mathrm{NH}_{4} \mathrm{OH}$ was used for purification $(56 \%$ yield, $0.053 \mathrm{mmol}) .{ }^{1} \mathrm{H} \mathrm{NMR}\left(400 \mathrm{MHz}, \mathrm{CDCl}_{3}\right): \delta 5.34\left(\mathrm{~s}(\mathrm{br}), 1 \mathrm{H}, \mathrm{RCH}=\mathrm{CR}_{2}\right), 4.82$ $\left(\mathrm{s}(\mathrm{br}), 1 \mathrm{H}, \mathrm{R}_{2} \mathrm{C}=\mathrm{CH}_{\mathrm{A}} \mathrm{H}_{\mathrm{B}}\right), 4.64\left(\mathrm{~s}(\mathrm{br}), 1 \mathrm{H}, \mathrm{R}_{2} \mathrm{C}=\mathrm{CH}_{\mathrm{A}} \mathbf{H}_{\mathbf{B}}\right), 3.25(\mathrm{ABq}, J=18.3 \mathrm{~Hz}, 2 \mathrm{H}$, $\left.\mathrm{NCH}_{2}\right), 2.87-2.75\left(\mathrm{~m}, 2 \mathrm{H}, \mathrm{NCH}_{2}\right), 2.26(\mathrm{~d}, J=13.2 \mathrm{~Hz}, 1 \mathrm{H}), 1.95-1.77(\mathrm{~m}, 6 \mathrm{H}), 1.77$ (s, $\left.3 \mathrm{H}, \mathrm{CH}_{3}\right), 1.66\left(\mathrm{~s}, 3 \mathrm{H}, \mathrm{CH}_{3}\right), 1.58-1.51(\mathrm{~m}, 1 \mathrm{H})$. Enantiomeric excess was determined by GLC analysis of the derived amide (23). See below for details.
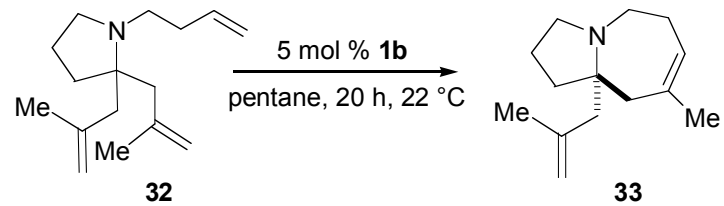

33. General procedure for ARCM was followed (pentane as solvent). Silica gel chromatography with 20:1 $\mathrm{CH}_{2} \mathrm{Cl}_{2}$ : $\mathrm{MeOH}$ washed with $1 \% \mathrm{v} / \mathrm{v}$ concentrated $\mathrm{NH}_{4} \mathrm{OH}$ was used for purification (quantitative yield, $0.042 \mathrm{mmol}$ ). ${ }^{1} \mathrm{H}$ NMR (400 $\mathrm{MHz}, \mathrm{CDCl}_{3}$ ):

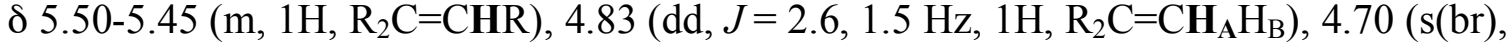
$\left.1 \mathrm{H}, \mathrm{R}_{2} \mathrm{C}=\mathrm{CH}_{\mathrm{A}} \mathbf{H}_{\mathbf{B}}\right), 2.97\left(\mathrm{ddd}, J=13.2,10.3,2.6 \mathrm{~Hz}, 1 \mathrm{H}, \mathrm{NCH}_{\mathrm{A}} \mathrm{H}_{\mathrm{B}} \mathrm{CH}_{2}\right), 2.90-2.77(\mathrm{~m}$, $\left.3 \mathrm{H}, \mathrm{NCH}_{2} \mathrm{CH}_{2}, \mathrm{NCH}_{\mathrm{A}} \mathbf{H}_{\mathbf{B}} \mathrm{CH}_{2}\right), 2.45(\mathrm{~d}, J=15.7 \mathrm{~Hz}, 1 \mathrm{H}), 2.42-2.32(\mathrm{~m}, 1 \mathrm{H}), 2.30(\mathrm{~d}, J=$ $13.6 \mathrm{~Hz}, 1 \mathrm{H}), 2.14(\mathrm{~d}, J=13.6 \mathrm{~Hz}, 1 \mathrm{H}), 2.12-2.05(\mathrm{~m}, 1 \mathrm{H}), 2.02(\mathrm{~d}, J=15.7 \mathrm{~Hz}, 1 \mathrm{H})$, 2.02-1.96 (m, 1H), 1.81 (s, 3H, CH $\left.\mathbf{H}_{3}\right), 1.75-1.68$ (m, 2H), 1.70 (s, 3H, CH $\left.\mathbf{H}_{3}\right), 1.46$ (ddd, $J$ $=11.7,9.5,9.5 \mathrm{~Hz}, 1 \mathrm{H})$; LRMS Calcd for $\mathrm{C}_{14} \mathrm{H}_{23} \mathrm{~N}(\mathrm{M}+\mathrm{H})$ : 206.19, Found: 206.19. Enantiomeric excess was determined by GLC analysis of the derived amide (20). 


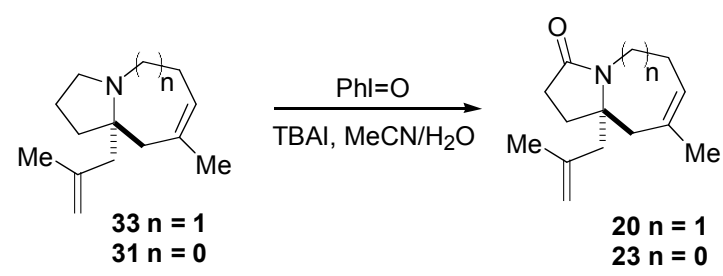

Enantiomeric excess determination of $\mathbf{3 1}$ and 33. Amines $\mathbf{3 1}$ and $\mathbf{3 3}$ was converted to the derived lactams (23 and $\mathbf{2 0}$ respectively) for ee analysis through an oxidation with iodosyl benzene (based on a literature procedure $)^{12}$ as follows: $\mathbf{3 3}(12 \mathrm{mg}, 0.06 \mathrm{mmol})$ was diluted with a 9:1 mixture of $\mathrm{MeCN} / \mathrm{H}_{2} \mathrm{O}(1.2 \mathrm{~mL}, 0.05 \mathrm{M})$ and tetrabutylammonium iodide $(4.5 \mathrm{mg}, 0.01 \mathrm{mmol}, 0.2$ equiv) was added. Iodosyl benzene (prepared by hydrolysis of $\mathrm{PhI}(\mathrm{OAc})_{2}$ according to a literature procedure, ${ }^{13} 29 \mathrm{mg}, 0.13 \mathrm{mmol}, 2.2$ equiv) was then added in one portion. After stirring for $\sim 3 \mathrm{~h}$, the mixture changes to a homogeneous orange/brown solution. Stirring was continued for $20 \mathrm{~h}$, and the mixture was then concentrated in vacuo. The resulting brown residue was dissolved in $\sim 1 \mathrm{~mL}$ $\mathrm{CHCl}_{3}$ and $\sim 1 \mathrm{~mL}$ of a $5 \%$ aqueous solution of $\mathrm{Na}_{2} \mathrm{~S}_{2} \mathrm{O}_{3}$. The layers are separated, and the aqueous fraction was washed with two $1 \mathrm{~mL}$ portions of $\mathrm{CHCl}_{3}$. The combined organic layers were washed with $3 \mathrm{~mL} \mathrm{H}_{2} \mathrm{O}$, dried with $\mathrm{Na}_{2} \mathrm{SO}_{3}$, filtered, and concentrated with a $\mathrm{N}_{2}$ purge. ${ }^{1} \mathrm{H}$ NMR analysis of the unpurified mixture indicated a complex mixture of oxidation products (the majority of the material was unreacted 33). Nonetheless a minor amount (1-3\%) of the desired amide 20 was present. Careful purification through a small plug of silica with $\mathrm{Et}_{2} \mathrm{O}$ (anisaldehyde for TLC visualization) afforded $\sim 0.5 \mathrm{mg}$ of $\mathbf{2 0}$. GC analysis indicated $40 \%$ ee of the product in comparison with racemic material. An analogous procedure was followed for the oxidation of $\mathbf{3 1}$ to afford trace quantities of $\mathbf{2 3}$ sufficient for GC analysis.

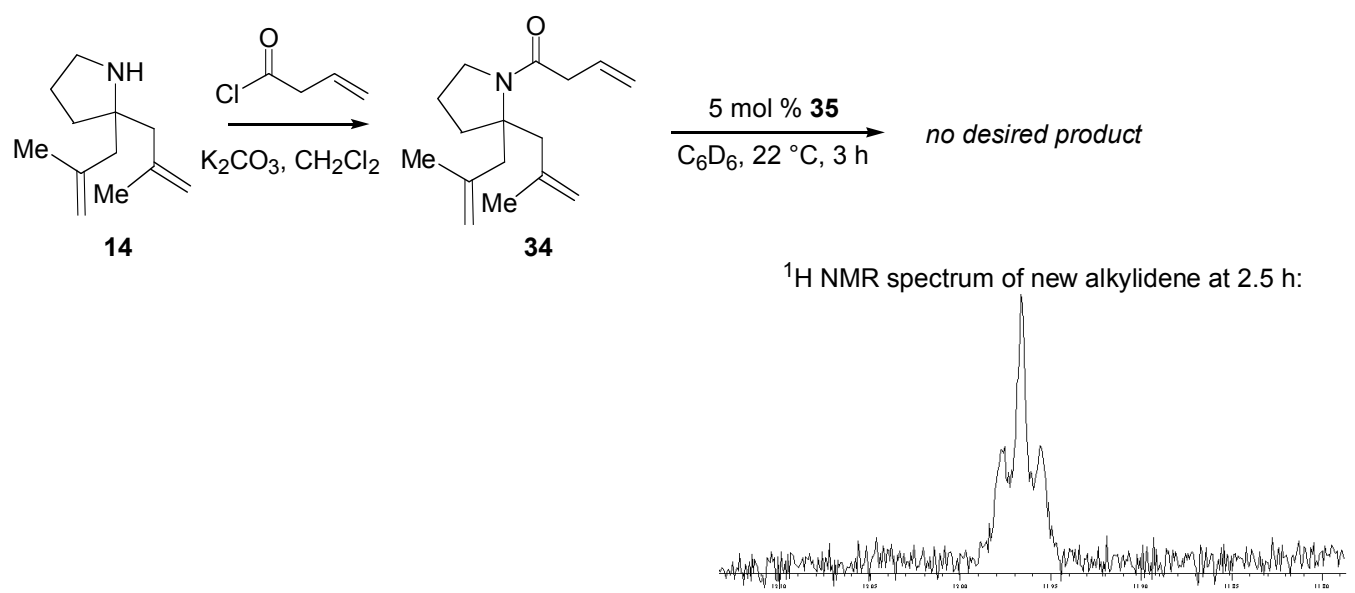

34. Amine 14 (108 mg, $0.60 \mathrm{mmol})$ was stirred with $\mathrm{K}_{2} \mathrm{CO}_{3}$ and 3-butenoyl chloride (84 $\mu \mathrm{L}, 0.72 \mathrm{mmol}$, prepared by the addition of 1 equiv oxalyl chloride followed by $5 \mathrm{~mol} \%$ DMF to a solution of vinyl acetic acid $\left(0.1 \mathrm{M}\right.$ in $\left.\mathrm{Et}_{2} \mathrm{O}\right)$ - after the gas evolution had ceased, the desired acid chloride was directly purified by simple distillation) in $\mathrm{CH}_{2} \mathrm{Cl}_{2}(3 \mathrm{~mL})$ for $12 \mathrm{~h}$. The mixture was then transferred to a separatory funnel and diluted with $\sim 5 \mathrm{~mL}$

(12) W.-J. Huang, O. V. Singh, C.-H. Chen, S.-Y. Chiou, S.-S Lee Helv. Chim. Act. 2002, 85, 1069-1078.

(13) Hypervalent Iodine in Organic Synthesis, A. Varvoglis; Academic Press; 1997, pp 13-14. 
$\mathrm{H}_{2} \mathrm{O}$ and $5 \mathrm{~mL} \mathrm{CH}_{2} \mathrm{Cl}_{2}$. The aqueous layer was washed three times with $5 \mathrm{~mL}$ portions of $\mathrm{CH}_{2} \mathrm{Cl}_{2}$. The combined organic fractions were dried over $\mathrm{MgSO}_{4}$, filtered and concentrated in vacuo. Purification by silica gel chromatography $\left(5: 1\right.$ pentane: $\left.\mathrm{Et}_{2} \mathrm{O}\right)$ gave $72 \mathrm{mg}$ of 34 (48\%). ${ }^{1} \mathrm{H} \mathrm{NMR}\left(400 \mathrm{MHz}, \mathrm{CDCl}_{3}\right.$ ): $\delta 5.97$ (ddt, $J=16.8,10.3,6.6 \mathrm{~Hz}, 1 \mathrm{H}$, $\left.\mathrm{RCH}=\mathrm{CH}_{2}\right), 5.16\left(\mathrm{~d}(\mathrm{br}), J=10.3 \mathrm{~Hz}, 1 \mathrm{H}, \mathrm{RCH}=\mathrm{CH}_{\mathrm{A}} \mathrm{H}_{\mathrm{B}}\right), 5.11$ (ddd, $J=17.2,3.3,1.8$ $\left.\mathrm{Hz}, \quad 1 \mathrm{H}, \quad \mathrm{RCH}=\mathrm{CH}_{\mathrm{A}} \mathbf{H}_{\mathbf{B}}\right), \quad 4.87-4.84 \quad\left(\mathrm{~m}, 2 \mathrm{H}, \mathrm{R}(\mathrm{Me}) \mathrm{C}=\mathrm{CH}_{\mathbf{A}} \mathrm{H}_{\mathrm{B}}\right), 4.69 \quad(\mathrm{~s}(\mathrm{br}), 2 \mathrm{H}$, $\left.\mathrm{R}(\mathrm{Me}) \mathrm{C}=\mathrm{CH}_{\mathrm{A}} \mathbf{H}_{\mathbf{B}}\right), 3.42\left(\mathrm{t}, J=7.0 \mathrm{~Hz}, 2 \mathrm{H}, \mathrm{NCH}_{2} \mathrm{R}\right), 3.14\left(\mathrm{~d}, J=13.6 \mathrm{~Hz}, 2 \mathrm{H}, \mathrm{OCCH}_{2} \mathrm{R}\right)$, $3.02\left(\mathrm{dt}, J=6.6,1.5 \mathrm{~Hz}, 2 \mathrm{H}, \mathrm{H}_{2} \mathrm{C}=\mathrm{CMeCH}_{\mathrm{A}} \mathrm{H}_{\mathrm{B}}\right), 2.28(\mathrm{~d}, J=13.2 \mathrm{~Hz}, 2 \mathrm{H}$, $\left.\mathrm{H}_{2} \mathrm{C}=\mathrm{CMeCH}_{\mathrm{A}} \mathbf{H}_{\mathbf{B}}\right), 1.97\left(\mathrm{t}, J=7.3 \mathrm{~Hz}, 2 \mathrm{H}, \mathrm{NCR}_{2} \mathrm{CH}_{2} \mathrm{R}\right), 1.76(\mathrm{tt}, J=14.3,7.0 \mathrm{~Hz}, 2 \mathrm{H}$, $\left.\mathrm{NCH}_{2} \mathrm{CH}_{2} \mathrm{CH}_{2} \mathrm{R}\right), 1.72\left(\mathrm{~s}, 6 \mathrm{H}, \mathrm{CH}_{3}\right)$.

Reaction of 34. In a $\mathrm{N}_{2}$ filled glove box, a threaded NMR tube was charged with 0.50 $\mathrm{mL}$ of a $0.1 \mathrm{M}$ solution of 34 in $\mathrm{C}_{6} \mathrm{D}_{6}$. Mo catalyst 35 (1.92 mg, $2.5 \mu \mathrm{mol}, 5 \mathrm{~mol} \%$ ) was added in one portion. A teflon cap was used to securely seal the tube and the reaction was monitored by ${ }^{1} \mathrm{H}$ NMR spectroscopy. None of the desired ring-closed product was observed and a new alkylidene resonance appeared $(11.97 \mathrm{ppm}, \mathrm{t}, J=4.5 \mathrm{~Hz})$ that persisted for several hours.

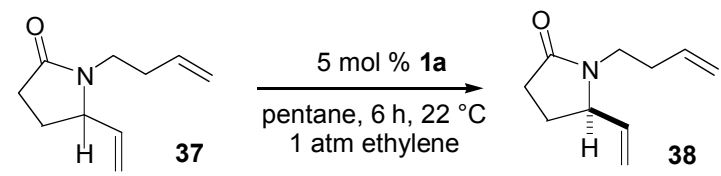

Kinetic resolution of 37 . Kinetic resolution substrate 37 was prepared according to published procedure. ${ }^{14}$ Kugelrohr distillation provided a clear oil for use in ARCM reactions. In a $\mathrm{N}_{2}$ filled dry box, a solution of pyrrolidinone $37\left(1.2 \mathrm{~mL}, 0.2 \mathrm{M}\right.$ in $\left.\mathrm{C}_{6} \mathrm{H}_{6}\right)$ was prepared in a 1 dram vial and sealed securely with a septum. A separate vial equipped with a stirbar was charged with a solution of Mo catalyst 1a $(7.6 \mathrm{mg}, 10 \mu \mathrm{mol}$ in $1.0 \mathrm{~mL} \mathrm{C}_{6} \mathrm{H}_{6}, 0.01 \mathrm{M}$ ). This vial was also sealed completely with a septum and teflon tape. Both vials were then removed from the glove box. The vial containing the catalyst solution was connected to a full ethylene (99.99\% anhydrous) balloon and purged for $\sim 2$ $\mathrm{s}$ through an exit needle. (The balloon apparatus consisted of a triple layered balloon connected to a short tube containing $\mathrm{Ca}_{2} \mathrm{SO}_{4}$, which was fitted with an 18 gauge needle. The entire apparatus was assembled in the glove box so as to exclude any air or moisture and then removed from the $\mathrm{N}_{2}$ atmosphere and purged three times with anhydrous ethylene before filling.) The vial containing the starting material was then connected to a $\mathrm{N}_{2}$ manifold and $1.0 \mathrm{~mL}$ of this solution $(33 \mathrm{mg}, 0.20 \mathrm{mmol}$ ) was transferred to the vial containing the catalyst solution via a gas-tight syringe that was first carefully purged with $\mathrm{N}_{2}$. The reaction was stirred under an ethylene atmosphere for $6 \mathrm{~h}$ before $0.5 \mathrm{~mL}$ of wet $\mathrm{Et}_{2} \mathrm{O}$ was added to quench the catalyst. The mixture was concentrated with a $\mathrm{N}_{2}$ purge to give a brown residue. ${ }^{1} \mathrm{H}$ NMR analysis indicated $66 \%$ conversion to the ring-closed product, indolizidinone 38 with $34 \%$ unreacted starting material. Isolation of the optically-enriched starting material 37 (9.9 mg, $0.06 \mathrm{mmol}, 30 \%$ yield) from the reaction mixture was accomplished with silica gel chromatography (EtOAc). GC analysis of 37 indicated $94 \%$ ee, corresponding to a $k_{\text {rel }}$ value of 8. Lactam 37: ${ }^{1} \mathrm{H}$ NMR $(400 \mathrm{MHz}$,

(14) Martin, S. F.; Chen, H-J.; Courtney, A. K.; Liao, Y.; Patzel, M.; Ramser, M. N.; Wagman, A. S. Tetrahedron 1996, 52, 7251-7264. 
$\mathrm{CDCl}_{3}$ ): $\delta 5.74$ (dddd, $\left.J=16.8,10.3,6.6,6.6 \mathrm{~Hz}, 1 \mathrm{H}, \mathrm{RCH}_{2} \mathrm{CH}=\mathrm{CH}_{2}\right), 5.66$, (ddd, $J=$ $\left.17.2,10.3,8.8 \mathrm{~Hz}, 1 \mathrm{H}, \mathrm{R}_{2} \mathrm{CHCH}=\mathrm{CH}_{2}\right), 5.24\left(\mathrm{~d}(\mathrm{br}), J=15.7 \mathrm{~Hz}, 1 \mathrm{H}, \mathrm{CH}=\mathrm{CH}_{\mathrm{A}} \mathrm{H}_{\mathrm{B}}\right), 5.21$ (d(br), $\left.J=9.2 \mathrm{~Hz}, 1 \mathrm{H}, \mathrm{CH}=\mathrm{CH}_{\mathrm{A}} \mathbf{H}_{\mathbf{B}}\right), 5.06\left(\mathrm{~d}(\mathrm{br}), J=17.6 \mathrm{~Hz}, 1 \mathrm{H}, \mathrm{CH}=\mathrm{CH}_{\mathrm{A}} \mathrm{H}_{\mathrm{B}}\right), 5.02$ (d(br), $\left.J=11.4 \mathrm{~Hz}, 1 \mathrm{H}, \mathrm{CH}=\mathrm{CH}_{\mathrm{A}} \mathbf{H}_{\mathbf{B}}\right), 4.05$ (ddd, $\left.J=8.1,8.1,5.5 \mathrm{~Hz}, 1 \mathrm{H}, \mathrm{NCH}\right), 3.60$ (ddd, $J=15.0,7.7,7.7 \mathrm{~Hz}, 1 \mathrm{H}, \mathrm{NCH}), 2.94$ (ddd, $J=13.6,8.1,5.9 \mathrm{~Hz}, 1 \mathrm{H}, \mathrm{NCH}), 2.45-$ $2.16(\mathrm{~m}, 5 \mathrm{H}), 1.73$ (dddd, $J=12.8,9.5,7.0,5.5 \mathrm{~Hz}, 1 \mathrm{H}) ;[\alpha]_{\mathrm{D}}+68.2\left(c=1, \mathrm{CHCl}_{3}\right)$.

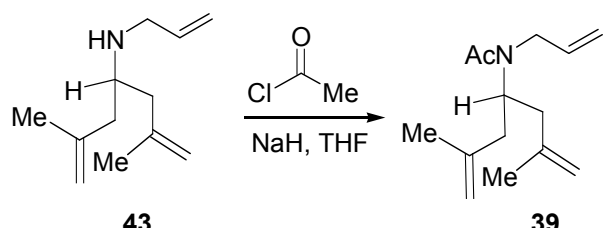

39. To a stirring solution of $\mathrm{NaH}(58.8 \mathrm{mg}, 2.45 \mathrm{mmol}, 1$ equiv) in $40 \mathrm{~mL}$ THF was added 43 (440 mg, $2.45 \mathrm{mmol}$, 1 equiv, see below for preparation) by cannula transfer with a total of $10 \mathrm{~mL}$ THF. The mixture was stirred for $1 \mathrm{~h}$ at which time it was cooled to $0{ }^{\circ} \mathrm{C}$ and acetylchloride (174 $\mu \mathrm{L}, 2.45 \mathrm{mmol}, 1$ equiv) was added dropwise via syringe. The mixture was then stirred for $12 \mathrm{~h}$. Water, $50 \mathrm{~mL}$, was added to react with any remaining $\mathrm{NaH}$ and the aqueous layer was separated. Three $40 \mathrm{~mL}$ portions of $\mathrm{Et}_{2} \mathrm{O}$ were used to extract the product from the aqueous layer. The combined organic fractions were dried over $\mathrm{MgSO}_{4}$, filtered and concentrated in vacuo to give an oil. ${ }^{1} \mathrm{H}$ NMR analysis indicated $\sim 65 \%$ conversion to the desired product 39. This compound was purified by silica gel chromatography $\left(20: 1 \mathrm{CH}_{2} \mathrm{Cl}_{2}: \mathrm{Et}_{2} \mathrm{O}\right.$ as the eluent) in $62 \%$ yield $(336 \mathrm{mg}, 1.52$ mmol). IR (neat): 3069 (s), 2974 (s), 2917 (s), 1797 (w), 1652 (s), 1413 (s), 1250 (m), $1036(\mathrm{~m}), 885(\mathrm{~s}) \mathrm{cm}^{-1}$; ${ }^{1} \mathrm{H}$ NMR $\left(400 \mathrm{MHz}, \mathrm{CDCl}_{3}, 25 \mathrm{C}\right), \sim 1: 3$ mixture of amide rotamers: $\delta 5.89$ (ddt, $J=16.1,10.3,5.9 \mathrm{~Hz}, 0.25 \mathrm{H}, \mathrm{RCH}=\mathrm{CH}_{2}$ ), 5.78 (ddt, $J=15.7$, 10.6, $\left.5.5 \mathrm{~Hz}, 0.75 \mathrm{H}, \mathrm{RCH}=\mathrm{CH}_{2}\right), 5.21-5.03\left(\mathrm{~m}, 2.75 \mathrm{H}, \mathrm{RCH}=\mathrm{CH}_{2}, \mathrm{NCHR}_{2}\right), 4.85$ (s(br), $\left.0.5 \mathrm{H}, \mathrm{R}_{2} \mathrm{C}=\mathrm{CH}_{\mathrm{A}} \mathrm{H}_{\mathrm{B}}\right), 4.76\left(\mathrm{~s}(\mathrm{br}), 1.5 \mathrm{H}, \mathrm{R}_{2} \mathrm{C}=\mathrm{CH}_{\mathrm{A}} \mathrm{H}_{\mathrm{B}}\right), 4.73\left(\mathrm{~s}(\mathrm{br}), 0.5 \mathrm{H}, \mathrm{R}_{2} \mathrm{C}=\mathrm{CH}_{\mathrm{A}} \mathbf{H}_{\mathrm{B}}\right)$, $4.70\left(\mathrm{~s}(\mathrm{br}), 1.5 \mathrm{H}, \mathrm{R}_{2} \mathrm{C}=\mathrm{CH}_{\mathrm{A}} \mathbf{H}_{\mathbf{B}}\right), 4.02\left(\mathrm{p}, J=6.6 \mathrm{~Hz}, 0.25 \mathrm{H}, \mathrm{NCHR}_{2}\right), 3.87(\mathrm{~d}(\mathrm{br}), J=5.9$ $\left.\mathrm{Hz}, 0.5 \mathrm{H}, \mathrm{NCH}_{2}\right), 3.75\left(\mathrm{dt}, J=5.1,1.5 \mathrm{~Hz}, 1.5 \mathrm{H}, \mathrm{NCH}_{2}\right), 2.25-2.14(\mathrm{~m}, 4 \mathrm{H}$, $\left.\mathrm{NCH}\left(\mathrm{CH}_{2} \mathrm{R}\right)_{2}\right), 2.13$ (s, 0.75H, $\left.\mathrm{CH}_{3} \mathrm{CO}\right), 2.03$ (s, 2.25H, $\left.\mathrm{CH}_{3} \mathrm{CO}\right), 1.75$ (s, 6H, $\mathrm{R}_{2} \mathrm{CCH}_{3}$ ); ${ }^{13} \mathrm{C}$ NMR $\left(100 \mathrm{MHz}, \mathrm{CDCl}_{3}\right): \delta 172.0,143.5,142.1,136.4,136.0,129.0,117.1,116.5$, 114.6, 113.2, 56.2, 50.3 (br), 47.0 (br), 44.6 (br), 42.2, 23.1, 23.0, 22.9, 22.6; HRMS Calcd for $\mathrm{C}_{14} \mathrm{H}_{23} \mathrm{NO}(\mathrm{M}+\mathrm{Na})$ : 244.1677, Found: 244.1672 .

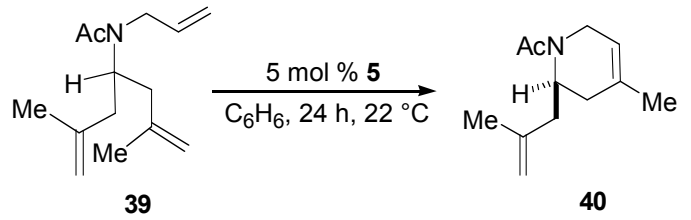

40. General procedure for ARCM was followed. Silica gel chromatography with 1:1 EtOAc:hexanes was used for purification (90\% yield, $0.22 \mathrm{mmol})$. IR (neat): $2962(\mathrm{~m})$, 2924 (m), 2855 (m), 1646 (s), 1426 (s), 1376 (w), 1237 (w), 891 (w) cm ${ }^{-1} ;{ }^{1} \mathrm{H}$ NMR (400 $\mathrm{MHz}, \mathrm{CDCl}_{3}, 25 \mathrm{C}$ ), 2:3 mixture of amide rotamers: $\delta 5.39\left(\mathrm{~s}(\mathrm{br}), 0.4 \mathrm{H}, \mathrm{R}_{2} \mathrm{C}=\mathrm{CHR}\right.$ ), $5.33\left(\mathrm{~s}(\mathrm{br}), 0.6 \mathrm{H}, \mathrm{R}_{2} \mathrm{C}=\mathrm{CHR}\right), 5.10\left(\mathrm{dd}, J=14.6,7.3 \mathrm{~Hz}, 0.6 \mathrm{H}, \mathrm{NCHR}_{2}\right), 4.83$ (s(br), $\left.0.4 \mathrm{H}, \mathrm{R}_{2} \mathrm{C}=\mathrm{CH}_{\mathrm{A}} \mathrm{H}_{\mathrm{B}}\right), 4.73\left(\mathrm{~s}(\mathrm{br}), 0.6 \mathrm{H}, \mathrm{R}_{2} \mathrm{C}=\mathrm{C} \mathbf{H}_{\mathrm{A}} \mathrm{H}_{\mathrm{B}}\right), 4.70\left(\mathrm{~s}(\mathrm{br}), 0.4 \mathrm{H}, \mathrm{R}_{2} \mathrm{C}=\mathrm{CH}_{\mathrm{A}} \mathbf{H}_{\mathrm{B}}\right)$, 4.65 (s(br), 0.6H, $\left.\mathrm{R}_{2} \mathrm{C}=\mathrm{CH}_{\mathrm{A}} \mathbf{H}_{\mathrm{B}}\right), 4.58$ (d(br), $\left.J=19.0 \mathrm{~Hz}, 0.4 \mathrm{H}, \mathrm{NCH}_{\mathrm{A}} \mathrm{H}_{\mathrm{B}} \mathrm{R}\right), 4.08$ (dd, $J$ 
$\left.=13.6,6.6 \mathrm{~Hz}, 0.4 \mathrm{H}, \mathrm{NCHR}_{2}\right), 3.91\left(\mathrm{~d}(\mathrm{br}), J=17.6 \mathrm{~Hz}, 0.6 \mathrm{H}, \mathrm{NCH}_{\mathrm{A}} \mathrm{H}_{\mathrm{B}} \mathrm{R}\right), 3.73(\mathrm{~d}(\mathrm{br}), J$ $\left.=17.6 \mathrm{~Hz}, 0.4 \mathrm{H}, \mathrm{NCH}_{\mathrm{A}} \mathbf{H}_{\mathrm{B}} \mathrm{R}\right), 3.39\left(\mathrm{~d}(\mathrm{br}), J=19.0 \mathrm{~Hz}, 0.4 \mathrm{H}, \mathrm{NCH}_{\mathrm{A}} \mathbf{H}_{\mathrm{B}} \mathrm{R}\right), 2.38-2.20(\mathrm{~m}$, 2.4H, $\left.\mathrm{NCHCH}_{2} \mathrm{R}\right), 2.11\left(\mathrm{~s}, 1.2 \mathrm{H}, \mathrm{CH}_{3}\right), 2.10-2.07\left(\mathrm{~m}, 0.6 \mathrm{H}, \mathrm{NCHCH}_{2} \mathrm{R}\right), 2.04(\mathrm{~s}, 1.8 \mathrm{H}$, $\left.\mathrm{CH}_{3}\right), 1.93-1.79\left(\mathrm{~m}, 1 \mathrm{H}, \mathrm{NCHCH}_{2} \mathrm{R}\right), 1.78\left(\mathrm{~s}, 1.8 \mathrm{H}, \mathrm{CH}_{3}\right), 1.77\left(\mathrm{~s}, 1.2 \mathrm{H}, \mathrm{CH}_{3}\right), 1.71$ (s(br), 3H, $\left.\mathrm{CH}_{3}\right) ;{ }^{13} \mathrm{C}$ NMR $\left(100 \mathrm{MHz}, \mathrm{CDCl}_{3}\right): \delta 170.1,144.1,142.5,132.5,130.1$, 118.0, 116.5, 114.7, 113.3, 51.1, 44.4, 42.7, 41.0, 40.6, 39.2, 33.9, 33.2, 30.4; HRMS Calcd for $\mathrm{C}_{12} \mathrm{H}_{19} \mathrm{NO}(\mathrm{M}+\mathrm{Na})$ : 216.1364, Found: 216.1367 .

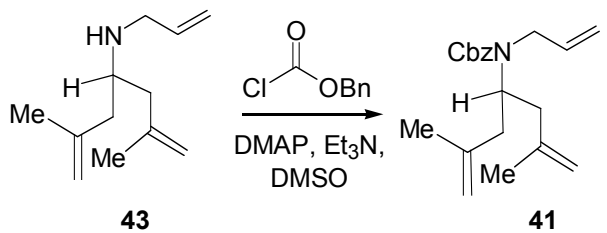

41. To a solution of 43 (400 mg, $2.23 \mathrm{mmol}, 1$ equiv, see below for preparation), $\mathrm{Et}_{3} \mathrm{~N}$ (0.66 mL, $8.92 \mathrm{mmol}, 4$ equiv, distilled over $\mathrm{CaH}_{2}$ ), and DMAP (55 mg, $0.45 \mathrm{mmol}, 0.2$ equiv) in DMSO $\left(2.23 \mathrm{~mL}\right.$, distilled over $\mathrm{CaH}_{2}$ ) was added $\mathrm{CbzCl}$ (carefully distilled to remove any $\mathrm{BnCl}$ resulting from decomposition, $0.64 \mathrm{~mL}, 4.46 \mathrm{mmol}, 2$ equiv) via syringe. The resulting mixture was heated to $50{ }^{\circ} \mathrm{C}$ and stirred for $12 \mathrm{~h}$. The reaction was then allowed to cool to ambient temperature and $\sim 10 \mathrm{~mL} \mathrm{H}_{2} \mathrm{O}$ followed by $\sim 10 \mathrm{~mL} \mathrm{Et}_{2} \mathrm{O}$ was added. The aqueous phase was separated and washed three times with $10 \mathrm{~mL}$ portions of $\mathrm{Et}_{2} \mathrm{O}$. The combined organic fractions were washed with $\sim 30 \mathrm{~mL}$ of a saturated aqueous solution of $\mathrm{NaHCO}_{3}$, dried over $\mathrm{MgSO}_{4}$, filtered, and concentrated in vacuo. ${ }^{1} \mathrm{H}$ NMR analysis of the unpurified reaction mixture indicated $\sim 60 \%$ conversion to the desired product. Purification with silica gel chromatography gave $335 \mathrm{mg}$ (1.07 mmol, 48\% yield) of 41. IR (neat): 3081 (m), 3031 (w), 2968 (m), $2924(\mathrm{~m}), 1697$ (s), 1646 (m), 1464 (s), 1407 (s), 1231 (s), $885(\mathrm{~m}) \mathrm{cm}^{-1} ;{ }^{1} \mathrm{H}$ NMR (400 MHz, DMSO-d 6,100 C): $\delta$ 7.37-7.28 (m, 5H, ArH), $5.78\left(\mathrm{ddt}, J=15.7,10.6,5.5 \mathrm{~Hz}, 1 \mathrm{H}, \mathrm{RCH}=\mathrm{CH}_{2}\right), 5.14(\mathrm{~d}$, $\left.J=17.2 \mathrm{~Hz}, \mathrm{RCH}=\mathrm{CH}_{\mathrm{A}} \mathrm{H}_{\mathrm{B}}\right), 5.06\left(\mathrm{~s}, 2, \mathrm{PhCH}_{2}\right), 5.02(\mathrm{~d}, J=10.3 \mathrm{~Hz}, 1 \mathrm{H}$, $\left.\mathrm{RCH}=\mathrm{CH}_{\mathrm{A}} \mathbf{H}_{\mathbf{B}}\right), 4.73$ (s(br), $\left.2 \mathrm{H}, \mathrm{R}_{2} \mathrm{C}=\mathrm{CH}_{\mathrm{A}} \mathrm{H}_{\mathrm{B}}\right), 4.68\left(\mathrm{~s}(\mathrm{br}), 2 \mathrm{H}, \mathrm{R}_{2} \mathrm{C}=\mathrm{CH}_{\mathrm{A}} \mathbf{H}_{\mathbf{B}}\right), 4.34$ (s(br), 1H, NCHR $), 3.74\left(\mathrm{~d}, J=5.1 \mathrm{~Hz}, 2 \mathrm{H}, \mathrm{NCH}_{2} \mathrm{R}\right), 2.29(\mathrm{dd}, J=14.3,9.9 \mathrm{~Hz}, 2 \mathrm{H}$, $\left.\mathrm{NCH}\left(\mathrm{CH}_{\mathbf{A}} \mathrm{H}_{\mathrm{B}} \mathrm{R}\right)_{2}\right), 2.12\left(\mathrm{dd}, J=14.3,5.1 \mathrm{~Hz}, 2 \mathrm{H}, \mathrm{NCH}\left(\mathrm{CH}_{\mathrm{A}} \mathbf{H}_{\mathbf{B}} \mathrm{R}\right)_{2}\right), 1.66\left(\mathrm{~s}, 3 \mathrm{H}, \mathrm{CH}_{3}\right)$; ${ }^{13} \mathrm{C}$ NMR $\left(100 \mathrm{MHz}, \mathrm{DMSO}-d_{6}, 100 \mathrm{C}\right): \delta 155.2,142.0,136.7,135.6,127.8,127.2$, 115.4, 112.1, 65.8, 52.7, 45.5, 40.5, 21.5; HRMS Calcd for $\mathrm{C}_{20} \mathrm{H}_{27} \mathrm{NO}_{2}(\mathrm{M}+\mathrm{Na})$ : 336.1939, Found: 336.1941.

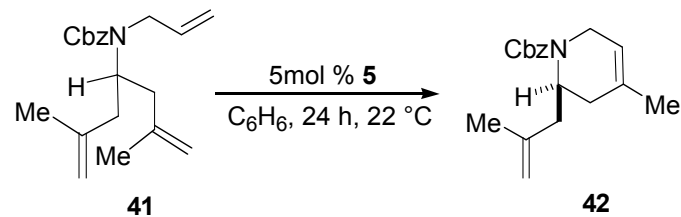

42. General procedure for ARCM was followed. Silica gel chromatography with $\mathrm{CH}_{2} \mathrm{Cl}_{2}$ was used for purification (98\% yield, $0.24 \mathrm{mmol}$ ). IR (neat): 3069 (w), 3031 (w), 2974 (m), 2917 (m), 2836 (m), 1709 (s), 1432 (s), 1344 (s), 1294 (s), 1224 (s), 1105 (s), 1048 (m), $891(\mathrm{~m}) \mathrm{cm}^{-1} ;{ }^{1} \mathrm{H}$ NMR (400 MHz, DMSO- $\left.d_{6}, 100{ }^{\circ} \mathrm{C}\right): \delta 7.37-7.28(\mathrm{~m}, 5 \mathrm{H}, \operatorname{ArH})$, 5.39 (s(br), 1H, $\left.\mathrm{R}_{2} \mathrm{C}=\mathrm{CHR}\right), 5.07\left(\mathrm{~s}, 2 \mathrm{H}, \mathrm{PhCH}_{2}\right), 4.71$ (s(br), 2H, $\left.\mathrm{R}_{2} \mathrm{C}=\mathrm{CH}_{\mathrm{A}} \mathrm{H}_{\mathrm{B}}\right), 4.65$ (s(br), $\left.1 \mathrm{H}, \mathrm{R}_{2} \mathrm{C}=\mathrm{CH}_{\mathrm{A}} \mathbf{H}_{\mathrm{B}}\right), 4.49\left(\mathrm{dd}, J=13.6,6.2 \mathrm{~Hz}, 1 \mathrm{H}, \mathrm{NCHR}_{2}\right), 4.11(\mathrm{~d}, J=17.9 \mathrm{~Hz}$, $\left.1 \mathrm{H}, \mathrm{NCH}_{\mathrm{A}} \mathrm{H}_{\mathrm{B}} \mathrm{R}\right), 3.52\left(\mathrm{~d}, J=17.6 \mathrm{~Hz}, 1 \mathrm{H}, \mathrm{NCH}_{\mathrm{A}} \mathbf{H}_{\mathrm{B}} \mathrm{R}\right), 2.30(\mathrm{~d}, J=16.8 \mathrm{~Hz}, 1 \mathrm{H}$, 
$\left.\mathrm{NCH}\left(\mathrm{CH}_{2}\right)_{2}\right), 2.20\left(\mathrm{dd}, J=13.6,8.4 \mathrm{~Hz}, 1 \mathrm{H}, \mathrm{NCH}\left(\mathrm{CH}_{2}\right)_{2}\right), 2.05(\mathrm{dd}, J=13.6,7.0 \mathrm{~Hz}$, $\left.1 \mathrm{H}, \mathrm{NCH}\left(\mathbf{C H}_{2}\right)_{2}\right), 1.79\left(\mathrm{~d}, J=17.2 \mathrm{~Hz}, \mathrm{NCH}\left(\mathrm{CH}_{2}\right)_{2}\right), 1.67\left(\mathrm{~s}(\mathrm{br}), 6 \mathrm{H}, \mathrm{CH}_{3}\right) ;{ }^{13} \mathrm{C} \mathrm{NMR}$ $\left(100 \mathrm{MHz}, \mathrm{DMSO}-d_{6}, 100{ }^{\circ} \mathrm{C}\right): \delta 155.5,143.2,142.7,137.1,130.1,128.5,128.3,128.0$, 117.0, 116.5, 113.3, 113.0, 67.0, 47.2, 46.7, 40.1, 33.1, 32.6, 29.8, 23.6, 22.2; HRMS Calcd for $\mathrm{C}_{18} \mathrm{H}_{23} \mathrm{NO}_{2}(\mathrm{M}+\mathrm{Na})$ : 308.1626, Found: 308.1622; $[\alpha]_{\mathrm{D}}-14.7\left(c=2, \mathrm{CHCl}_{3}\right)$.

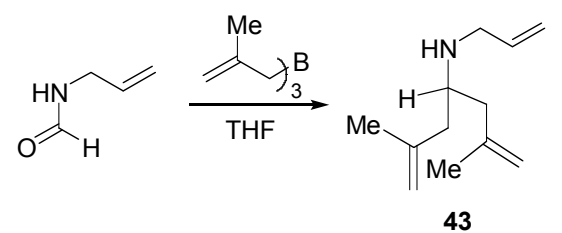

43. A reductive alkylation of allylformamide ${ }^{15}$ with trimethallylborane was performed according to the procedure for the synthesis of $\mathbf{1 4}$ (see above). Secondary amine $\mathbf{4 3}$ is obtained in $71 \%$ yield $(8.5 \mathrm{mmol})$ after silica gel chromatography (4:1 DCM:hexanes washed with $1 \% \mathrm{v} / \mathrm{v}$ concentrated $\mathrm{NH}_{4} \mathrm{OH}$, Dragendorff's reagent for TLC visualization). The amine can be further purified by Kugelrohr distillation under vacuum to give a clear oil. IR (neat): 3087 (m), 2980 (m), 2936 (s), $1652(\mathrm{~m}), 1457(\mathrm{~m}), 1381(\mathrm{~m}) \mathrm{cm}^{-1} ;{ }^{1} \mathrm{H}$ NMR (400 MHz, $\mathrm{CDCl}_{3}$ ): $\delta 5.85\left(\mathrm{dddd}, J=16.8,10.3,6.2,6.2 \mathrm{~Hz}, 1 \mathrm{H}, \mathrm{RCH}=\mathrm{CH}_{2}\right.$ ), 5.15 (ddd, $\left.J=17.2,3.3,1.5 \mathrm{~Hz}, 1 \mathrm{H}, \mathrm{RCH}=\mathrm{CH}_{\mathrm{A}} \mathrm{H}_{\mathrm{B}}\right), 5.08(\mathrm{~d}(\mathrm{br}), J=10.3 \mathrm{~Hz}, 1 \mathrm{H}$, $\left.\mathrm{RCH}=\mathrm{CH}_{\mathrm{A}} \mathbf{H}_{\mathbf{B}}\right), 4.81\left(\mathrm{~s}(\mathrm{br}), 2 \mathrm{H}, \mathrm{R}_{2} \mathrm{C}=\mathrm{CH}_{\mathrm{A}} \mathrm{H}_{\mathrm{B}}\right), 4.75\left(\mathrm{~s}(\mathrm{br}), 2 \mathrm{H}, \mathrm{R}_{2} \mathrm{C}=\mathrm{CH}_{\mathrm{A}} \mathbf{H}_{\mathbf{B}}\right), 3.26(\mathrm{~d}$, $J=5.9 \mathrm{~Hz}, 2 \mathrm{H}, \mathrm{NCH}_{2} \mathrm{R}$ ), 2.82 (dddd, $\left.J=6.6,6.6,6.6,6.6 \mathrm{~Hz}, 1 \mathrm{H}, \mathrm{NCHR}_{2}\right), 2.15$ (dd, $J=$ 13.9, 7.3 Hz, 2H, NCHCH $\left.\mathrm{H}_{\mathrm{B}} \mathrm{R}\right), 2.07$ (dd, $\left.J=13.9,6.2 \mathrm{~Hz}, 2 \mathrm{H}, \mathrm{NCHCH}_{\mathrm{A}} \mathbf{H}_{\mathbf{B}} \mathrm{R}\right), 1.72(\mathrm{~s}$, $\left.6 \mathrm{H}, \mathrm{CH}_{3}\right), 1.28$ (s(br), 1H, NH); ${ }^{13} \mathrm{C}$ NMR (100 MHz, $\left.\mathrm{CDCl}_{3}\right): \delta$ 143.5, 137.2, 116.0, 113.1, 52.0, 50.1, 43.4, 22.6; HRMS Calcd for $\mathrm{C}_{12} \mathrm{H}_{21} \mathrm{~N}(\mathrm{M}+\mathrm{H})$ : 180.1752, Found: 180.1751 .

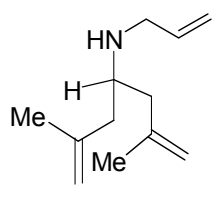

43
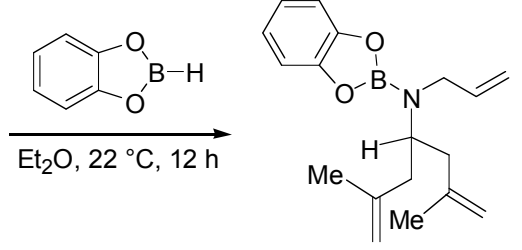

53

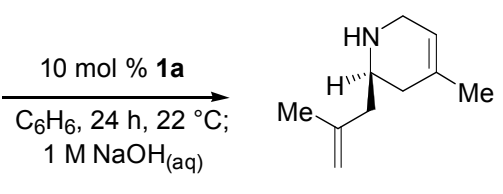

44

Amine-borane complex 53. To a stirring solution of amine $43(50.3 \mathrm{mg}, 0.280 \mathrm{mmol})$ in $\mathrm{Et}_{2} \mathrm{O}(2.8 \mathrm{~mL})$ under an inert atmosphere was added $29.9 \mathrm{~mL}$ catechol borane (freshly distilled from $\mathrm{CaH}_{2}, 0.280 \mathrm{mmol}$ ). The resulting solution was stirred for $12 \mathrm{~h}$. At this time, the solvent was removed by a stream of $\mathrm{N}_{2}$ and then in vacuo. The complex was azeotroped with $\mathrm{C}_{6} \mathrm{H}_{6}$ (see materials section). ${ }^{1} \mathrm{H}$ NMR analysis revealed that all starting material had been consumed, however, the signals for complex $\mathbf{5 3}$ were not well resolved.

(15) Prepared according to literature procedure. See: McManus, S. P.; Carroll, J. T.; Piiman Jr., C. U.; J. Org. Chem. 1970, 35, 3768-3774. 
44. General procedure for ARCM was followed with a modified work-up: the unpurified reaction mixture was diluted with an equal volume of $1 \mathrm{M}$ aqueous $\mathrm{NaOH}$ solution and stirred for $1 \mathrm{~h}$. The aqueous phase was then separated and washed three times with $\mathrm{CH}_{2} \mathrm{Cl}_{2}$. The combined organic extracts were dried with $\mathrm{Na}_{2} \mathrm{SO}_{4}$, filtered and concentrated almost to dryness (product is volatile). Silica gel chromatography with 20:1 $\mathrm{CH}_{2} \mathrm{Cl}_{2}: \mathrm{MeOH}$ washed with $2 \% \mathrm{v} / \mathrm{v}$ concentrated $\mathrm{NH}_{4} \mathrm{OH}$ was used for purification $(50 \%$ yield, 0.027 mmol). IR (neat): 3270 (w), 3075 (m), 2917 (s), 1659 (m), 1451 (s), 1381 (m), 890 (s) cm ${ }^{-1} ;{ }^{1} \mathrm{H}$ NMR (400 MHz, $\mathrm{CDCl}_{3}$ ): $\delta 5.41$ (s(br), $\left.1 \mathrm{H} \mathrm{RCH}=\mathrm{CR}_{2}\right), 4.83$ (s(br), $1 \mathrm{H}, \mathrm{R}_{2} \mathrm{C}=\mathrm{CH}_{\mathrm{A}} \mathrm{H}_{\mathrm{B}}$ ), 4.78 ( $\mathrm{s}(\mathrm{br}), 1 \mathrm{H}, \mathrm{R}_{2} \mathrm{C}=\mathrm{CH}_{\mathrm{A}} \mathbf{H}_{\mathbf{B}}$ ), 3.35 (s(br), 2H, $\mathrm{NCH}_{2} \mathrm{R}$ ), 2.85 (dddd, $\left.J=9.5,7.7,5.9,4.4 \mathrm{~Hz}, 1 \mathrm{H}, \mathrm{NCHR}_{2}\right), 2.17-2.13\left(\mathrm{~m}, 2 \mathrm{H}, \mathrm{NCHCH}_{2} \mathrm{R}\right), 1.90-1.72(\mathrm{~m}, 3 \mathrm{H}$, $\left.\mathrm{NCHCH}_{2} \mathrm{R}, \mathrm{NH}\right), 1.74\left(\mathrm{~s}, 3 \mathrm{H}, \mathrm{CH}_{3}\right), 1.67\left(\mathrm{~s}, 3 \mathrm{H}, \mathrm{CH}_{3}\right) ;{ }^{13} \mathrm{C} \mathrm{NMR}\left(100 \mathrm{MHz}, \mathrm{CDCl}_{3}\right)$ : $\delta 142.9,132.9,120.1,112.9,50.4,45.6,45.3,37.3,23.4,22.5$; HRMS Calcd for $\mathrm{C}_{10} \mathrm{H}_{17} \mathrm{~N}$ $(\mathrm{M}+\mathrm{H})$ : 152.1439, Found: 152.1436; $[\alpha]_{\mathrm{D}}+7.7(c=1)$. Enantiomeric excess was determined by GLC analysis of the derived acetamide (40).

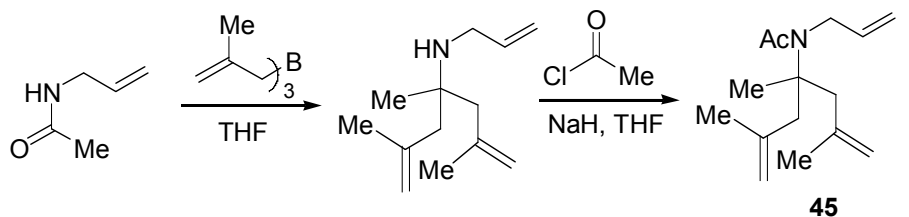

45. Alkylation of allyl acetamide with trimethallyl borane was preformed according the procedure for the preparation of 2,2-bis-(2-methyl-allyl)-pyrrolidine $\mathbf{1 4}$ with the following modification: trimethallyl borane was added to a $-78{ }^{\circ} \mathrm{C}$ solution of allyl acetamide in THF. After the addition, the mixture was allowed to warm to ambient temperature and was subsequently stirred for $12 \mathrm{~h}$ before quench with $\mathrm{MeOH}$ and $3 \mathrm{M}$ $\mathrm{NaOH}$. Allyl-[1,3-dimethyl-1-(2-methyl-allyl)-but-3-enyl]-amine was obtained after purification by silica gel chromatography $\left(15: 1\right.$ pentane: $\mathrm{Et}_{2} \mathrm{O}$ washed with $1 \% \mathrm{v} / \mathrm{v}$ concentrated $\left.\mathrm{NH}_{4} \mathrm{OH}\right)$ in $47 \%$ yield $(7 \mathrm{mmol})$. This secondary amine was then converted to the acetamide under the conditions reported for the synthesis of 39. By this method, 45 was obtained in $44 \%$ yield after silica gel chromatography $\left(20: 1 \mathrm{CH}_{2} \mathrm{Cl}_{2}: \mathrm{Et}_{2} \mathrm{O}\right)$. IR (neat): 3087 (w), 2980 (m), 1671 (s), 1470 (m), 1401 (s), 1212 (m) cm ${ }^{-1} ;{ }^{1} \mathrm{H}$ NMR (400 MHz, $\left.\mathrm{CDCl}_{3}\right): \delta 5.79\left(\mathrm{ddt}, J=17.2,11.0,5.5 \mathrm{~Hz}, 1 \mathrm{H}, \mathrm{RCH}=\mathrm{CH}_{2}\right), 5.19-5.12(\mathrm{~m}, 2 \mathrm{H}$, $\left.\mathrm{RCH}=\mathrm{CH}_{2}\right), 4.87\left(\mathrm{dq}, J=2.5,1.1 \mathrm{~Hz}, 2 \mathrm{H}, \mathrm{R}_{2} \mathrm{C}=\mathrm{CH}_{\mathrm{A}} \mathrm{H}_{\mathrm{B}}\right), 4.68-4.67(\mathrm{~m}, 2 \mathrm{H}$, $\left.\mathrm{R}_{2} \mathrm{C}=\mathrm{CH}_{\mathrm{A}} \mathbf{H}_{\mathrm{B}}\right), 3.83\left(\mathrm{dt}, J=5.5,1.8 \mathrm{~Hz}, 2 \mathrm{H}, \mathrm{NCH}_{2} \mathrm{R}\right), 3.34(\mathrm{~d}, J=13.2 \mathrm{~Hz}, 2 \mathrm{H}$, $\left.\mathrm{NC}(\mathrm{Me}) \mathrm{CH}_{\mathrm{A}} \mathrm{H}_{\mathrm{B}} \mathrm{R}\right), 2.17\left(\mathrm{~d}, J=13.2 \mathrm{~Hz}, 2 \mathrm{H}, \mathrm{NCMeCH}_{\mathrm{A}} \mathbf{H}_{\mathrm{B}} \mathrm{R}\right), 2.07$ (s, 3H, CH $\mathbf{C H}_{3} \mathrm{CO}$ ), 1.73 (s, 6H, $\left.\mathrm{CR}_{2} \mathrm{CH}_{3}\right), 1.32$ (s, 3H, $\left.\mathrm{NCR}_{2} \mathrm{CH}_{3}\right) ;{ }^{13} \mathrm{C} \mathrm{NMR}\left(100 \mathrm{MHz}, \mathrm{CDCl}_{3}\right): \mathrm{d} 172.3$, 143.0, 136.2, 116.3, 115.4, 63.3, 49.8, 45.8, 25.5, 25.0, 24.6; HRMS Calcd for $\mathrm{C}_{15} \mathrm{H}_{25} \mathrm{NO}$ $(\mathrm{M}+\mathrm{H}): 236.2014$, Found: 236.2011.

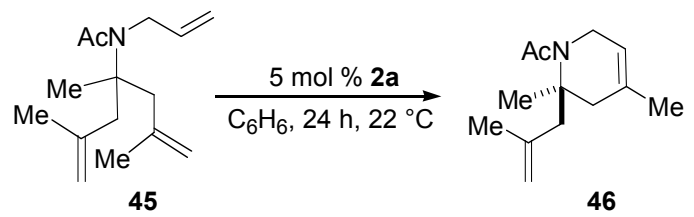

46. General procedure for ARCM was followed. Silica gel chromatography with 15:1 $\mathrm{CH}_{2} \mathrm{Cl}_{2}: \mathrm{Et}_{2} \mathrm{O}$ was used for purification $(63 \%$ yield, $0.140 \mathrm{mmol})$. IR (neat): $3068(\mathrm{~m})$, 2968 (s), 2923 (s), 2861 (m), 1646 (s), 1694 (s), 1243 (m), 1168 (m), 897 (s) cm ${ }^{-1} ;{ }^{1} \mathrm{H}$ 
NMR (400 MHz, $\left.\mathrm{CDCl}_{3}\right): \delta 5.53\left(\mathrm{~s}(\mathrm{br}), 1 \mathrm{H}, \mathrm{RCH}=\mathrm{CR}_{2}\right), 4.83\left(\mathrm{~s}, 1 \mathrm{H}, \mathrm{R}_{2} \mathrm{C}=\mathrm{CH}_{\mathrm{A}} \mathrm{H}_{\mathrm{B}}\right), 4.66$ $\left(\mathrm{s}, 1 \mathrm{H}, \mathrm{R}_{2} \mathrm{C}=\mathrm{CH}_{\mathrm{A}} \mathbf{H}_{\mathrm{B}}\right), 3.85\left(\mathrm{dd}, J=15.7,4.8 \mathrm{~Hz}, 1 \mathrm{H}, \mathrm{NCH}_{\mathrm{A}} \mathrm{H}_{\mathrm{B}} \mathrm{R}\right), 3.68(\mathrm{dd}, J=15.7,1.5$ $\left.\mathrm{Hz}, 1 \mathrm{H}, \mathrm{NCH}_{\mathrm{A}} \mathbf{H}_{\mathrm{B}} \mathrm{R}\right), 3.02\left(\mathrm{~d}, J=13.6 \mathrm{~Hz}, 1 \mathrm{H}, \mathrm{NCR}_{2} \mathrm{CH}_{\mathrm{A}} \mathrm{H}_{\mathrm{B}} \mathrm{R}\right), 2.34(\mathrm{~d}, J=14.6 \mathrm{~Hz}, 1 \mathrm{H}$, $\left.\mathrm{NCR}_{2} \mathrm{CH}_{\mathrm{A}} \mathrm{H}_{\mathrm{B}} \mathrm{R}\right), 2.30\left(\mathrm{~d}, J=13.6 \mathrm{~Hz}, 1 \mathrm{H}, \mathrm{NCR}_{2} \mathrm{CH}_{\mathrm{A}} \mathbf{H}_{\mathrm{B}} \mathrm{R}\right), 2.06$ (s, 3H, CH $\left.\mathrm{CH}_{3} \mathrm{CO}\right), 1.87$ $\left(\mathrm{d}, J=15.7 \mathrm{~Hz}, 1 \mathrm{H}, \mathrm{NCR}_{2} \mathrm{CH}_{\mathrm{A}} \mathbf{H}_{\mathrm{B}} \mathrm{R}\right), 1.74\left(\mathrm{~s}, 3 \mathrm{H}, \mathrm{CH}_{3}\right), 1.49\left(\mathrm{~s}, 3 \mathrm{H}, \mathrm{CH}_{3}\right) ;{ }^{13} \mathrm{C} \mathrm{NMR}$ $\left(100 \mathrm{MHz}, \mathrm{CDCl}_{3}\right): \delta 171.0,143.3,136.2,117.6,114.9,58.7,45.2,44.8,42.5,26.9,25.5$, 24.2, 23.0; HRMS Calcd for $\mathrm{C}_{13} \mathrm{H}_{21} \mathrm{NO}(\mathrm{M}+\mathrm{H}): 208.1701$, Found: 208.1698.

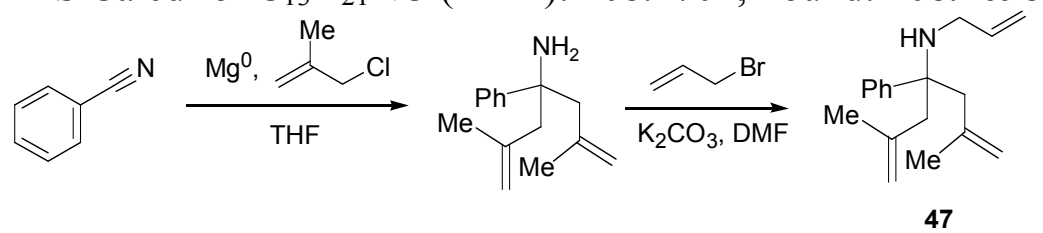

47. Amine 47 was prepared according to the procedure for the synthesis of 49 (see below). ${ }^{1} \mathrm{H}$ NMR (400 MHz, $\mathrm{CDCl}_{3}$ ): $\delta$ 7.46-7.43 (m, 2H), 7.32-7.27 (m, 2H), 7.21-7.16 (m, 1H), 6.01-5.92 (tdd, $J=16.0,10.0,5.6 \mathrm{~Hz}, 1 \mathrm{H}), 5.27-5.21$ (ddd, $J=17.2,2.0,1.6$ $\mathrm{Hz}, 1 \mathrm{H}), 5.10-5.06$ (ddd, $J=10.4,1.6,1.2 \mathrm{~Hz}, 1 \mathrm{H}), 4.78-4.76$ (td, $J=4,1.6 \mathrm{~Hz}, 2 \mathrm{H})$, $4.61(\mathrm{~s}, 2 \mathrm{H}), 3.22-3.19(\mathrm{td}, J=5.6,1.2 \mathrm{~Hz}, 2 \mathrm{H}), 2.68-2.64(\mathrm{~d}, J=14 \mathrm{~Hz}, 2 \mathrm{H}), 2.54-2.50$ $(\mathrm{d}, J=14 \mathrm{~Hz}, 1 \mathrm{H}), 1.57(\mathrm{~s}, 1 \mathrm{H}), 1.42(\mathrm{~s}, 6 \mathrm{H}) \cdot{ }^{13} \mathrm{C} \mathrm{NMR}\left(100 \mathrm{MHz}, \mathrm{CDCl}_{3}\right): \delta 145.9$, $142.6,137.4,128.0,127.0,126.3,115.2,114.7,61.1,46.2,45.1,24.9$.

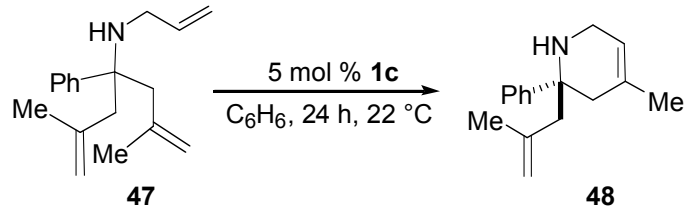

48. General procedure for ARCM was followed. Silica gel chromatography with $2.5 \%$ $\mathrm{MeOH}$ in $\mathrm{CH}_{2} \mathrm{Cl}_{2}$ was used for purification (95\% yield, $0.039 \mathrm{mmol}$ ). IR (neat): 3383 (br), 2961 (m), 2917 (s), 2848 (m), 2369 (w); ${ }^{1} \mathrm{H}$ NMR (400 MHz, CDCl ${ }_{3}$ ): $\delta$ 7.38-7.28 (m, 4H), 7.23-7.18 (m, 1H), $5.30(\mathrm{~s}, 1 \mathrm{H}), 4.77(\mathrm{~s}, 1 \mathrm{H}), 4.60(\mathrm{~s}, 1 \mathrm{H}), 3.32-3.24(\mathrm{~d}, 20 \mathrm{~Hz}$, 1H), 3.06-2.98 (d, $20 \mathrm{~Hz}, 1 \mathrm{H}), 2.55-2.24(\mathrm{~m}, 4 \mathrm{H}), 1.75(\mathrm{~s}, 3 \mathrm{H}), 1.25(\mathrm{~s}, 3 \mathrm{H}) ;{ }^{13} \mathrm{C} \mathrm{NMR}$ $\left(100 \mathrm{MHz}, \mathrm{C}_{6} \mathrm{D}_{6}\right): \delta 146.0,142.8,131.4,128.4,127.3,126.8,121.5,115.2,57.5,51.9$, 42.6, 39.2, 24.9, 24.0; HRMS calcd for $\mathrm{C}_{16} \mathrm{H}_{21} \mathrm{~N}$ : $228.1752(\mathrm{M}+\mathrm{H})$, Found 228.1747. Enantiomeric excess was determined by GLC analysis of the derived acetamide. Acetyprotected 48: ${ }^{1} \mathrm{H}$ NMR $\left(400 \mathrm{MHz}, \mathrm{CDCl}_{3}\right): \delta$ 7.24-7.14 (m, 5H), $5.60(\mathrm{~s}, 1 \mathrm{H}), 4.92(\mathrm{~s}$, $1 \mathrm{H}), 4.77(\mathrm{~s}, 1 \mathrm{H}), 4.20-3.56(\mathrm{~m}, 3 \mathrm{H}), 2.87-2.80(\mathrm{t}, J=12 \mathrm{~Hz}, 2 \mathrm{H}), 2.20-2.16(\mathrm{~d}, J=16$ $\mathrm{Hz}, 1 \mathrm{H}), 2.03(\mathrm{~s}, 3 \mathrm{H}), 1.63(\mathrm{~s}, 3 \mathrm{H}), 1.54(\mathrm{~s}, 3 \mathrm{H})$.

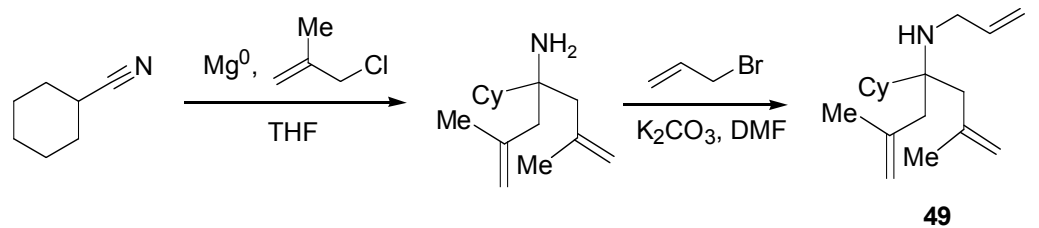

49. The primary amine required for the synthesis of 49 was prepared according to a modified literature procedure. ${ }^{16}$ A three-neck $500 \mathrm{~mL}$ round-bottom flask, with attached addition funnel and reflux condenser, was charged with cyclohexanecarbonitrile (3.30

(16) Henze, H. R.; Allen, B. B.; Leslie W. B. J. Am. Chem. Soc. 1944, 65, 87-89 
$\mathrm{mL}, 27.5 \mathrm{mmol})$, magnesium turnings $(2.50 \mathrm{~g}, 103 \mathrm{mmol})$, and THF (200 $\mathrm{mL})$. The resulting mixture was allowed to stir at ambient temperature. Methallyl chloride (6.80 $\mathrm{mL}, 68.7 \mathrm{mmol})$ and THF (68 mL) were transferred to the addition funnel. Then $10 \mathrm{~mL}$ of the methallyl chloride solution was slowly added to the mixture of cyclohexanecarbonitrile with heating (heat gun) to initiate the reaction (during initiation the solution became olive green and was exothermic). At this point the reaction mixture was no longer heated and the remainder of the methallyl chloride solution was added slowly over one hour. The reaction mixture was then refluxed. After $16 \mathrm{~h}$ the reaction mixture was cooled to $0{ }^{\circ} \mathrm{C}$ and quenched with a saturated aqueous solution of $\mathrm{NH}_{4} \mathrm{Cl}$ $(100 \mathrm{~mL})$. The aqueous layer was separated and the organic layer was concentrated to remove the majority of the THF. The crude reaction mixture was diluted with $\mathrm{Et}_{2} \mathrm{O}$ (100 $\mathrm{mL})$, washed with $\mathrm{H}_{2} \mathrm{O}(3 \times 50 \mathrm{~mL})$, dried $\left(\mathrm{MgSO}_{4}\right)$, filtered and concentrated in vacuo. 1-cyclohexyl-3-methyl-1-(2-methyl-allyl)-but-3-enylamine was purified by silica gel chromatography $\left(20: 1 \mathrm{CH}_{2} \mathrm{Cl}_{2}: \mathrm{MeOH}\right)$ to give the primary amine as a yellow oil $(5.0 \mathrm{~g}$, $83 \%$ yield). ${ }^{1} \mathrm{H}$ NMR (400 $\left.\mathrm{MHz}, \mathrm{CDCl}_{3}\right): \delta 4.93-4.91(\mathrm{dd}, J=2.4,1.2 \mathrm{~Hz}, 2 \mathrm{H})$, 4.73-4.71 (dd, $J=2.4,1.2 \mathrm{~Hz}, 2 \mathrm{H}), 2.22-2.18$ (d, $J=13.2 \mathrm{~Hz}, 2 \mathrm{H}), 2.07-2.04$ (d, $J=$ $13.2 \mathrm{~Hz}, 2 \mathrm{H}), 1.81(\mathrm{~s}, 6 \mathrm{H}), 1.79-1.6(\mathrm{~m}, 2 \mathrm{H}), 1.40-1.00(\mathrm{~m}, 9 \mathrm{H})$. A $50 \mathrm{~mL}$ round-bottom flask was charged with 1-cyclohexyl-3-methyl-1-(2-methyl-allyl)-but-3-enylamine (0.50 $\mathrm{g}, 2.3 \mathrm{mmol})$, potassium carbonate $(0.31 \mathrm{~g}, 2.3 \mathrm{mmol})$, DMF $(13 \mathrm{~mL})$, and allyl bromide $(0.20 \mathrm{~mL}, 2.3 \mathrm{mmol})$. The resulting solution was allowed to stir at ambient temperature for $16 \mathrm{~h}$. The reaction mixture was diluted with $\mathrm{Et}_{2} \mathrm{O}(50 \mathrm{~mL})$, washed with $\mathrm{H}_{2} \mathrm{O}(6 \times 25$ $\mathrm{mL})$, dried $\left(\mathrm{MgSO}_{4}\right)$, filtered and concentrated in vacuo. The crude product was purified by silica gel chromatography $\left(20: 1 \mathrm{CH}_{2} \mathrm{Cl}_{2}: \mathrm{MeOH}\right)$ to give amine 49 as a yellow oil $(0.40$ g, 68\% yield). ${ }^{1} \mathrm{H}$ NMR (400 $\left.\mathrm{MHz}, \mathrm{CDCl}_{3}\right): \delta 5.96-5.86(\mathrm{tdd}, J=18.0,11.6,6.0 \mathrm{~Hz}$, $1 \mathrm{H}), 5.22-5.20$ (ddd, $J=17.2,3.6,2.0 \mathrm{~Hz}, 1 \mathrm{H}), 5.17-5.15(\mathrm{ddd}, J=17.2,3.6,2.0 \mathrm{~Hz}$, $1 \mathrm{H}), 5.04-5.03$ (dd, $J=3.6,2.0 \mathrm{~Hz}, 1 \mathrm{H}), 5.02-5.00$ (dd, $J=3.6,2.0 \mathrm{~Hz}, 1 \mathrm{H}), 4.85-4.84$ $(\mathrm{dd}, J=2.4,1.2 \mathrm{~Hz}, 2 \mathrm{H}), 4.75-4.74(\mathrm{dd}, J=2.4,1.2 \mathrm{~Hz}, 2 \mathrm{H}), 3.24-3.21(\mathrm{~m}, 2 \mathrm{H}), 2.16$ (s, $3 \mathrm{H}), 1.86(\mathrm{~s}, 3 \mathrm{H}), 1.82-1.09(\mathrm{~m}, 11 \mathrm{H}) .{ }^{13} \mathrm{C} \mathrm{NMR}\left(100 \mathrm{MHz}, \mathrm{CDCl}_{3}\right): \delta 144.4,137.7$, $114.6,114.5,60.6,45.9,42.9,27.6,27.5,27.0,25.5$.

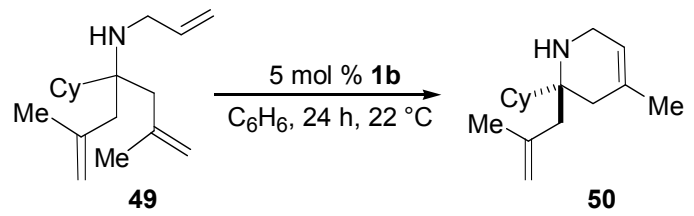

50. General procedure for ARCM was followed. Silica gel chromatography with 1:1 $\mathrm{Et}_{2} \mathrm{O}$ :hexane was used for purification (56\% yield, $\left.0.076 \mathrm{mmol}\right)$. IR (neat): $2924(\mathrm{~m})$, 2855 (m), 1734 (w), $1639(\mathrm{w}) ;{ }^{1} \mathrm{H}$ NMR (400 MHz, $\left.\mathrm{CDCl}_{3}\right): \delta 5.38(\mathrm{~s}, 1 \mathrm{H}), 4.92(\mathrm{~s}, 1 \mathrm{H})$, $4.72(\mathrm{~s}, 1 \mathrm{H}), 3.40-3.21(\mathrm{dd}, J=41.6,16.8 \mathrm{~Hz}, 2 \mathrm{H}), 2.32-1.98(\mathrm{~m}, 4 \mathrm{H}), 1.84(\mathrm{~s}, 3 \mathrm{H}), 1.66$ $(\mathrm{s}, 3 \mathrm{H}), 1.48-0.87(\mathrm{~m}, 11 \mathrm{H}) ;{ }^{13} \mathrm{C}$ NMR $\left(100 \mathrm{MHz}, \mathrm{C}_{6} \mathrm{D}_{6}\right): \delta 131.3,127.9,120.0,114.6$, $42.8,42.0,40.7,36.1,27.5,27.4,27.3,27.2,25.5,24.1$; HRMS calcd for $\mathrm{C}_{16} \mathrm{H}_{27} \mathrm{~N}$ : 234.2222 $(\mathrm{M}+\mathrm{H})$, Found 234.2224. Enantiomeric excess was determined by GLC analysis of the derived acetamide. Acetyl-protected 50: ${ }^{1} \mathrm{H}$ NMR $\left(400 \mathrm{MHz}, \mathrm{CDCl}_{3}\right): \delta$ $5.46(\mathrm{~s}, 1 \mathrm{H}), 4.73(\mathrm{~s}, 1 \mathrm{H}), 4.65(\mathrm{~s}, 1 \mathrm{H}), 3.82-3.00(\mathrm{~m}, 2 \mathrm{H}), 3.52-3.46(\mathrm{~m}, 1 \mathrm{H}), 2.75-2.65$ $(\mathrm{m}, 1 \mathrm{H}), 2.20-2.10(\mathrm{~m}, 1 \mathrm{H}), 2.10(\mathrm{~s}, 3 \mathrm{H}), 2.10-2.00(\mathrm{~m}, 1 \mathrm{H}), 1.78(\mathrm{~s}, 3 \mathrm{H}), 1.62(\mathrm{~s}, 3 \mathrm{H})$, $1.45-0.82(\mathrm{~m}, 11 \mathrm{H})$. 


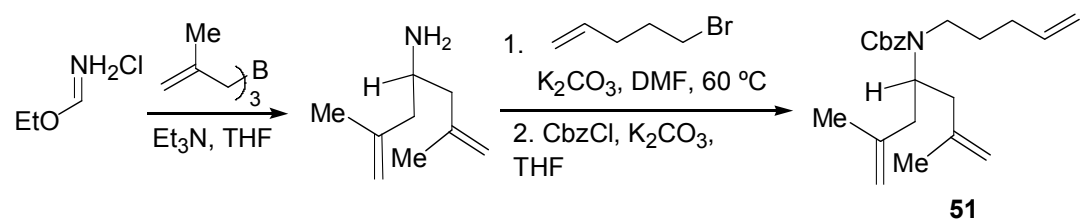

51. Alkylation of ethyl formimidate $\cdot \mathrm{HCl}$ was preformed according the procedure for the preparation of 2,2-bis-(2-methyl-allyl)-pyrrolidine $\mathbf{1 4}$ with the following modification: after the addition of trimethallyl borane, 1.2 equiv of $\mathrm{Et}_{3} \mathrm{~N}$ (distilled from $\mathrm{CaH}_{2}$ ) was added (with respect to substrate). The desired primary amine was obtained in 57\% yield (6 mmol) after purification with silica gel chromatography $\left(30: 1 \mathrm{CH}_{2} \mathrm{Cl}_{2}: \mathrm{MeOH}\right.$ washed with $4 \% \mathrm{v} / \mathrm{v}$ concentrated $\mathrm{NH}_{4} \mathrm{OH}$ ). IR (neat): $3075(\mathrm{~m}), 2936(\mathrm{~s}), 1646(\mathrm{~m}), 1448(\mathrm{~m})$, $1382(\mathrm{~m}), 904(\mathrm{~s}) \mathrm{cm}^{-1} ;{ }^{1} \mathrm{H}$ NMR $\left(400 \mathrm{MHz}, \mathrm{CDCl}_{3}\right): \delta 4.82\left(\mathrm{~s}(\mathrm{br}), 2 \mathrm{H}, \mathrm{R}_{2} \mathrm{C}=\mathrm{CH}_{\mathrm{A}} \mathrm{H}_{\mathrm{B}}\right)$, 4.77 (s(br), $2 \mathrm{H}, \mathrm{R}_{2} \mathrm{C}=\mathrm{CH}_{\mathrm{A}} \mathbf{H}_{\mathbf{B}}$ ), 3.08 (dddd, $J=8.8,8.8,4.4,4.4 \mathrm{~Hz}, 1 \mathrm{H}, \mathrm{NCHR}_{2}$ ), 2.13 (dd, $J=13.6,4.4 \mathrm{~Hz}, 2 \mathrm{H}, \mathrm{RCH}_{\mathrm{A}} \mathrm{CH}_{\mathrm{B}} \mathrm{R}$ ), 1.95 (dd, $J=14.3,9.5 \mathrm{~Hz}, 2 \mathrm{H}, \mathrm{RCH}_{\mathrm{A}} \mathrm{CH} \mathbf{H}_{\mathrm{B}} \mathrm{R}$ ), 1.74 (s, $\left.6 \mathrm{H}, \mathrm{CH}_{3}\right), 1.18$ (s(br), $\left.2 \mathrm{H}, \mathrm{NH}_{2}\right) ;{ }^{13} \mathrm{C}$ NMR (100 MHz, CDCl $): \delta 143.4,112.8$, 46.8, 46.0, 22.4; HRMS Calcd for $\mathrm{C}_{9} \mathrm{H}_{17} \mathrm{~N}(\mathrm{M}+\mathrm{H})$ : 140.1439, Found: 140.1436. 3Methyl-1-(2-methyl-allyl)-but-3-enyl]-amine (500 mg, $3.59 \mathrm{mmol}, 1$ equiv) was diluted with $36 \mathrm{~mL}$ DMF (dried over 4 A molecular sieves). Potassium carbonate (546 mg, 3.95 mmol, 1.1 equiv) was added followed by 5-bromo-1-pentene $(0.47 \mathrm{~mL}, 3.95 \mathrm{mmol}, 1.1$ equiv, distilled from $\mathrm{CaH}_{2}$ ). The reaction was sealed and stirred at $60{ }^{\circ} \mathrm{C}$ for 3 days. At this time, the mixture was diluted with $\sim 40 \mathrm{~mL} \mathrm{H} \mathrm{H}_{2} \mathrm{O}$ and an equal portion of $\mathrm{CH}_{2} \mathrm{Cl}_{2}$. The aqueous layer was separated and washed with three additional portions of $\mathrm{CH}_{2} \mathrm{Cl}_{2}$. The organic fractions were combined, washed with three $200 \mathrm{~mL}$ portions of a saturated aqueous solution of $\mathrm{NaCl}$, dried over $\mathrm{Na}_{2} \mathrm{SO}_{4}$, filtered and concentrated to an oil. Purification with silica gel chromatography delivered the desired secondary amine [3methyl-1-(2-methyl-allyl)-but-3-enyl]-pent-4-enyl-amine in 74\% yield $(549 \mathrm{mg}, 2.64$ mmol). IR (neat): 3075 (m), 2930 (s), 1652 (m), 1451 (m), 1130 (w), $904(\mathrm{~s}) \mathrm{cm}^{-1} ;{ }^{1} \mathrm{H}$ NMR (400 MHz, $\mathrm{CDCl}_{3}$ ): $\delta 5.80$ (ddt, $J=17.1,10.1,6.7 \mathrm{~Hz}, 1 \mathrm{H}, \mathrm{RCH}=\mathrm{CH}_{2}$ ), 5.01 (ddd, $\left.J=17.1,3.6,1.5 \mathrm{~Hz}, 1 \mathrm{H}, \mathrm{RCH}=\mathrm{CH}_{\mathrm{A}} \mathrm{H}_{\mathrm{B}}\right), 4.95\left(\mathrm{~d}(\mathrm{br}), J=10.4 \mathrm{~Hz}, 1 \mathrm{H}, \mathrm{RCH}=\mathrm{CH}_{\mathrm{A}} \mathbf{H}_{\mathbf{B}}\right)$, $4.82\left(\mathrm{~s}(\mathrm{br}), 2 \mathrm{H}, \mathrm{R}_{2} \mathrm{C}=\mathrm{CH}_{\mathbf{A}} \mathrm{H}_{\mathrm{B}}\right), 4.75\left(\mathrm{~s}(\mathrm{br}), 2 \mathrm{H}, \mathrm{R}_{2} \mathrm{C}=\mathrm{CH}_{\mathrm{A}} \mathbf{H}_{\mathbf{B}}\right), 2.75(\mathrm{p}, J=6.4 \mathrm{~Hz}, 1 \mathrm{H}$, $\left.\mathrm{NCHR}_{2}\right), 2.61\left(\mathrm{t}, J=7.0 \mathrm{~Hz}, 2 \mathrm{H}, \mathrm{NCH}_{2} \mathrm{R}\right), 2.17-2.04(\mathrm{~m}, 6 \mathrm{H}), 1.72\left(\mathrm{~s}, 6 \mathrm{H}, \mathrm{CH}_{3}\right), 1.56(\mathrm{p}$, $J=7.3 \mathrm{~Hz}, 2 \mathrm{H}), 1.24(\mathrm{~s}(\mathrm{br}), 1 \mathrm{H}, \mathrm{NH}) ;{ }^{13} \mathrm{C} \mathrm{NMR}\left(100 \mathrm{MHz}, \mathrm{CDCl}_{3}\right): \delta 143.5,138.5$, 114.8, 112.9, 53.1, 47.0, 43.5, 31.6, 29.5, 22.6; HRMS Calcd for $\mathrm{C}_{14} \mathrm{H}_{25} \mathrm{~N}(\mathrm{M}+\mathrm{H})$ : 208.2065, Found: 208.2069. This material was protected under standard conditions (2 equiv $\mathrm{CbzCl}$, 2.2 equiv $\mathrm{K}_{2} \mathrm{CO}_{3}$, THF, $0{ }^{\circ} \mathrm{C}$ to ambient temperature, $1 \mathrm{~h}$ ) to give $\mathbf{5 1}$ in $92 \%$ yield. IR (neat): 3087 (w), 2974 (m), 2930 (m), 1690 (s), 1658 (w), 1413 (m), 1274 (m), $1224(\mathrm{~m}) \mathrm{cm}^{-1}$; ${ }^{1} \mathrm{H}$ NMR $\left(400 \mathrm{MHz}, \mathrm{CDCl}_{3}, 25 \mathrm{C}\right), \sim 2: 3$ mixture of amide rotamers: $\delta$ 7.37-7.27 (m, 5H, ArH), 5.82 (ddt, $J=16.8,10.3,6.6 \mathrm{~Hz}, 0.4 \mathrm{H}, \mathrm{RCH}=\mathrm{CH}_{2}$ ), 5.75 (ddt, $\left.J=16.8,10.3,6.6 \mathrm{~Hz}, 0.6 \mathrm{H}, \mathrm{RCH}=\mathrm{CH}_{2}\right), 5.13\left(\mathrm{~s}, 2 \mathrm{H}, \mathrm{PhCH}_{2}\right), 5.06-4.94(\mathrm{~m}, 2 \mathrm{H}$, $\left.\mathrm{RCH}=\mathrm{CH}_{2}\right), 4.76\left(\mathrm{~s}(\mathrm{br}), 2 \mathrm{H}, \mathrm{R}_{2} \mathrm{C}=\mathrm{CH}_{2}\right), 4.71\left(\mathrm{~s}, 1 \mathrm{H}, \mathrm{R}_{2} \mathrm{C}=\mathrm{CH}_{2}\right), 4.67\left(\mathrm{~s}, 1 \mathrm{H}, \mathrm{R}_{2} \mathrm{C}=\mathrm{C} \mathbf{H}_{2}\right)$, 4.45 (s(br), 0.6H, NCHR 2 ), 4.33 (s(br), 0.4H, NCHR 2 ), 3.12-3.02 (m, 2H, NCH $\left.\mathbf{N}_{2} \mathrm{R}\right), 2.30-$ $1.97(\mathrm{~m}, 7 \mathrm{H}), 1.75\left(\mathrm{~s}, 3 \mathrm{H}, \mathrm{CH}_{3}\right), 1.72-1.59(\mathrm{~m}, 1 \mathrm{H}), 1.65\left(\mathrm{~s}, 3 \mathrm{H}, \mathrm{CH}_{3}\right) ;{ }^{13} \mathrm{C}$ NMR $(100$ $\mathrm{MHz}, \mathrm{CDCl}_{3}$ ): $\delta 156.4$ (br), 142.8, 142.4, 138.2, 138.0, 137.3, 137.0, 128.5 (br), 128.3, 128.1, 127.9, 127.7, 115.1, 115.0, 113.2, 112.9, 67.1, 66.8, 53.3 (br), 43.4 (br), 41.8, 41.5, 31.6 (br), 28.8, 29.3, 28.4, 22.4; HRMS Calcd for $\mathrm{C}_{22} \mathrm{H}_{31} \mathrm{NO}_{2}(\mathrm{M}+\mathrm{H})$ : 342.2433, Found: 342.2434 . 


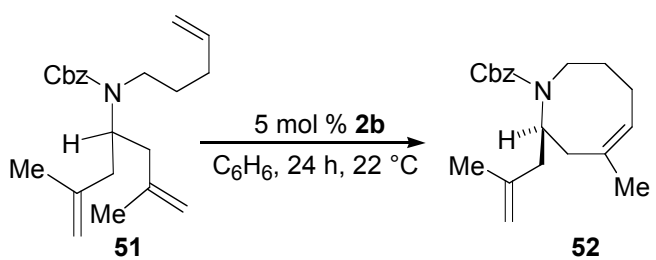

52. General procedure for ARCM was followed. Silica gel chromatography with 10:1 hexane: $\mathrm{Et}_{2} \mathrm{O}$ was used for purification (95\% yield, $\left.0.95 \mathrm{mmol}\right)$. IR (neat): $3062(\mathrm{w}), 3031$ (w), $2974(\mathrm{~m}), 2930(\mathrm{~m}), 1703$ (s), 1627 (m), 1501 (m), 1269 (m), 1168 (s), $998(\mathrm{~m}) \mathrm{cm}^{-1}$; ${ }^{1} \mathrm{H}$ NMR $\left(400 \mathrm{MHz}, \mathrm{CDCl}_{3}, 25 \mathrm{C}\right), \sim 2: 3$ mixture of amide rotamers: $\delta$ 7.37-7.27 $(\mathrm{m}, 5 \mathrm{H}$, ArH), $5.54\left(\mathrm{dd}, J=7.3,7.3 \mathrm{~Hz}, 0.4 \mathrm{H}, \mathrm{R}_{2} \mathrm{C}=\mathrm{CHR}\right), 5.48(\mathrm{dd}, J=8.1,8.1 \mathrm{~Hz}, 0.6 \mathrm{H}$, $\left.\mathrm{R}_{2} \mathrm{C}=\mathrm{CHR}\right), 5.12\left(\mathrm{~s}, 1.2 \mathrm{H}, \mathrm{PhCH}_{2}\right), 5.09\left(\mathrm{ABq}, J=12.5 \mathrm{~Hz}, 0.8 \mathrm{H}, \mathrm{PhCH}_{2}\right), 4.73-4.64$ $\left(\mathrm{m}, 2.6 \mathrm{H}, \mathrm{R}_{2} \mathrm{C}=\mathrm{CH}_{2}, \mathrm{NCHR}_{2}\right.$ ), 4.52-4.44 (m, 0.4H, NCHR $), 3.64$ (ddd, $J=14.3,2.9,2.9$ $\left.\mathrm{Hz}, 0.4 \mathrm{H}, \mathrm{NCH}_{\mathrm{A}} \mathrm{H}_{\mathrm{B}} \mathrm{R}\right), 3.55$ (ddd, $\left.J=15.0,3.3,3.3 \mathrm{~Hz}, 0.6 \mathrm{H}, \mathrm{NCH}_{\mathrm{A}} \mathrm{H}_{\mathrm{B}} \mathrm{R}\right), 2.88-2.76(\mathrm{~m}$, $\left.1 \mathrm{H}, \mathrm{NCH}_{\mathrm{A}} \mathbf{H}_{\mathrm{B}} \mathrm{R}\right), 2.36-2.22(\mathrm{~m}, 2 \mathrm{H}), 2.13(\mathrm{dd}, J=13.9,5.9 \mathrm{~Hz}, 1 \mathrm{H}), 2.08-1.91(\mathrm{~m}, 3 \mathrm{H})$, 1.87-1.79 (m, 1H), 1.77 (s, 3H, CH $\left.\mathbf{H}_{3}\right), 1.66\left(\mathrm{~s}, 1.8 \mathrm{H}, \mathrm{CH}_{3}\right), 1.63\left(\mathrm{~s}, 1.2 \mathrm{H}, \mathrm{CH}_{3}\right), 1.53-1.44$ $(\mathrm{m}, 1 \mathrm{H}) ;{ }^{13} \mathrm{C}$ NMR $\left(100 \mathrm{MHz}, \mathrm{CDCl}_{3}\right): \delta 156.9,156.2,142.9,142.3,137.5,137.2,135.5$, $134.9,128.5,127.9,127.8,127.6,127.2,126.3,113.3,112.8,66.83,66.81,52.7,52.6$, 43.3, 42.35, 42.26, 42.1, 36.7, 36.5, 29.9, 29.1, 27.9, 26.9, 26.5, 24.3, 23.8, 22.30, 22.27; HRMS Calcd for $\mathrm{C}_{20} \mathrm{H}_{27} \mathrm{NO}_{2}(\mathrm{M}+\mathrm{Na})$ : 336.1939, Found: 336.1927. Enantiomeric excess was determined by GLC analysis of the derived trifluoroacetamide, obtained by acylation of 54. See below for details.

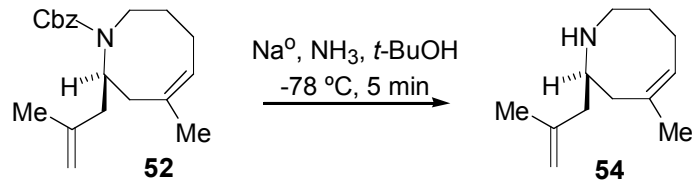

54. A $15 \mathrm{~mL}$ two- necked flask containing a teflon stirbar was charged with $\sim 11 \mathrm{mg}$ metallic $\mathrm{Na}(0.6 \mathrm{mmol}, 6$ equiv., washed with hexanes). The flask was then fitted with a Dewar condenser and purged with $\mathrm{N}_{2}$. After cooling the flask and condenser to $-78{ }^{\circ} \mathrm{C}$, $\sim 4 \mathrm{~mL}$ of $\mathrm{NH}_{3}(0.03 \mathrm{M})$ was condensed onto the metallic $\mathrm{Na}$ with stirring to produce a deep blue solution. A solution of Cbz-protected azocine $52(33.1 \mathrm{mg}, 0.106 \mathrm{mmol})$ and $60 \mu \mathrm{L}$ of $t-\mathrm{BuOH}$ in $\sim 1 \mathrm{~mL}$ of $\mathrm{Et}_{2} \mathrm{O}$ was rapidly added to the stirring solution via cannula. An additional $1 \mathrm{~mL}$ of $\mathrm{Et}_{2} \mathrm{O}$ was used for complete transfer. The resulting mixture should remain deep blue. On occasion, the color changed to white or pale yellow and a second portion of metallic $\mathrm{Na}$ was added to again produce a deep blue solution. The reaction was allowed to stir for $5 \mathrm{~min}$ at $-78^{\circ} \mathrm{C}$. At this time, solid $\mathrm{NH}_{4} \mathrm{Cl}$ was added in small portions until the blue color dissipated. The mixture was then allowed to gradually warm to ambient temp (without stirring). After bubbling (of $\mathrm{NH}_{3}$ ) had ceased, the mixture was transferred to a separatory funnel with $\sim 15 \mathrm{~mL} \mathrm{CH}_{2} \mathrm{Cl}_{2}$ and $\sim 10 \mathrm{~mL} \mathrm{H}_{2} \mathrm{O}$. The aqueous layer was washed $2 \mathrm{x}$ with $10 \mathrm{~mL}$ portions of $\mathrm{CH}_{2} \mathrm{Cl}_{2}$. The combined organic extracts were dried over $\mathrm{Na}_{2} \mathrm{SO}_{4}$, filtered and concentrated under reduced pressure. Analysis by ${ }^{1} \mathrm{H}$ NMR indicated $>90 \%$ conversion of starting material. Purification by silica gel chromatography in 30:1 $\mathrm{CH}_{2} \mathrm{Cl}_{2}: \mathrm{MeOH}$ washed with $2 \% \mathrm{v} / \mathrm{v}$ concentrated $\mathrm{NH}_{4} \mathrm{OH}$ (Dragendorff's reagent for TLC visualization) afforded $17.5 \mathrm{mg}(0.098 \mathrm{mmol}, 88 \%)$ of pure azocine 54. IR (neat): 3367 (w), 3075 (w), 2920 (s), 2851 (m), 1648 (w), 1443 (m), 1375 (w), 1133 (m), 903 (m) $\mathrm{cm}^{-1} ;{ }^{1} \mathrm{H}$ NMR (400 MHz, $\left.\mathrm{CDCl}_{3}\right): \delta 5.43$ (dd, $J=8.1,8.1$ 
$\left.\mathrm{Hz}, 1 \mathrm{H}, \mathrm{R}_{2} \mathrm{C}=\mathrm{CHR}\right), 4.83$ (s(br), $\left.1 \mathrm{H}, \mathrm{R}_{2} \mathrm{C}=\mathrm{CH}_{\mathbf{A}} \mathrm{H}_{\mathrm{B}}\right), 4.76\left(\mathrm{~s}(\mathrm{br}), 1 \mathrm{H}, \mathrm{R}_{2} \mathrm{C}=\mathrm{CH}_{\mathrm{A}} \mathbf{H}_{\mathrm{B}}\right), 2.92$ (ddd, $J=14.3,4.4,4.4 \mathrm{~Hz}, 1 \mathrm{H}, \mathrm{NCH}_{\mathrm{A}} \mathrm{H}_{\mathrm{B}} \mathrm{R}$ ), 2.70 (dddd, $J=9.2,9.2,5.1,2.2 \mathrm{~Hz}, 1 \mathrm{H}$, $\mathrm{NCHR}_{2}$ ), 2.45 (ddd, $\left.J=13.9,10.3,3.3,1 \mathrm{H}, \mathrm{NCH}_{\mathrm{A}} \mathbf{H}_{\mathrm{B}} \mathrm{R}\right), 2.36-2.26(\mathrm{~m}, 1 \mathrm{H}), 2.29$ (dd, $J=$ $13.2,8.8 \mathrm{~Hz}, 1 \mathrm{H}), 2.17(\mathrm{dd}, J=13.9,9.2 \mathrm{~Hz}, 1 \mathrm{H}), 2.07(\mathrm{dd}, J=13.6,4.8 \mathrm{~Hz}, 1 \mathrm{H}), 2.00-$ $1.93(\mathrm{~m}, 1 \mathrm{H}), 1.87$ (dd, $J=13.6,2.2 \mathrm{~Hz}, 1 \mathrm{H}), 1.75$ (d(br), $\left.0.7 \mathrm{~Hz}, 3 \mathrm{H}, \mathrm{CH}_{3}\right), 1.73$ (s(br), 3H, $\mathrm{CH}_{3}$ ), 1.64 (dddd, $\left.J=15.0,15.0,4.4,4.4 \mathrm{~Hz}, 1 \mathrm{H}\right), 1.54$ (s(br), 1H, NH), 1.35 (dddd, $J=12.5,12.5,8.4,4.0 \mathrm{~Hz}, 1 \mathrm{H}) ;{ }^{13} \mathrm{C} \mathrm{NMR}\left(100 \mathrm{MHz}, \mathrm{CDCl}_{3}\right): \delta 143.5,136.4,124.7$, 113.2, 55.5, 45.8, 45.4, 10.2, 32.2, 25.1, 24.9, 22.2; HRMS Calcd for $\mathrm{C}_{12} \mathrm{H}_{21} \mathrm{~N}(\mathrm{M}+\mathrm{H})$ : 180.1752, Found: 180.1752 .

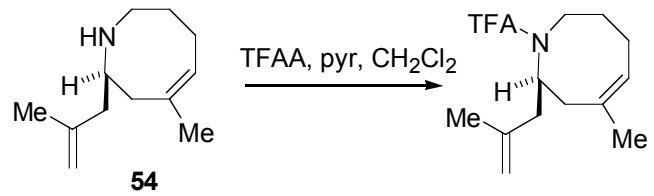

Enantiomeric excess determination of 52. Deprotection of 52 was performed as reported above. Standard conditions (pyridine, TFAA, $\mathrm{CH}_{2} \mathrm{Cl}_{2}$ ) were then employed for the synthesis of trifluoroacetamide-protected 54, obtained in $84 \%$ yield after silica gel chromatography (9:1 hexanes:EtOAc). TFA-protected 54: ${ }^{1} \mathrm{H}$ NMR (400 $\mathrm{MHz}, \mathrm{CDCl}_{3}$, $25 \mathrm{C}), 1: 4$ mixture of amide rotamers: $\delta 5.58\left(\mathrm{dd}, J=8.4,8.4 \mathrm{~Hz}, 0.8 \mathrm{H}, \mathrm{R}_{2} \mathrm{C}=\mathrm{CHR}\right), 5.54$ (dd, $\left.J=7.7,7.7 \mathrm{~Hz}, 0.2 \mathrm{H}, \mathrm{R}_{2} \mathrm{C}=\mathrm{CHR}\right), 5.05$ (s(br), 0.2H, NCHR $\left.\mathbf{N}_{2}\right), 4.87$ (s(br), 0.8H, $\mathrm{R}_{2} \mathrm{C}=\mathrm{CH}_{\mathrm{A}} \mathrm{H}_{\mathrm{B}}$ ), 4.77 ( $\left.\mathrm{s}(\mathrm{br}), 1 \mathrm{H}, \mathrm{R}_{2} \mathrm{C}=\mathrm{CH}_{\mathrm{A}} \mathbf{H}_{\mathrm{B}}\right), 4.66\left(\mathrm{~s}(\mathrm{br}), 0.2 \mathrm{H}, \mathrm{R}_{2} \mathrm{C}=\mathrm{CH}_{\mathrm{A}} \mathrm{H}_{\mathrm{B}}\right), 4.12$ (s(br), 0.8H, NCHR $), 3.87$ (d(br), $\left.J=13.9 \mathrm{~Hz}, 0.8 \mathrm{H}, \mathrm{NCH}_{\mathrm{A}} \mathrm{H}_{\mathrm{B}} \mathrm{R}\right), 3.71$ (d(br), $J=16.5$ $\left.\mathrm{Hz}, 0.2 \mathrm{H}, \mathrm{NCH}_{\mathrm{A}} \mathrm{H}_{\mathrm{B}} \mathrm{R}\right), 3.12\left(\mathrm{~m}, 0.2 \mathrm{H}, \mathrm{NCH}_{\mathrm{A}} \mathbf{H}_{\mathrm{B}} \mathrm{R}\right), 3.04$ (dd, $J=12.8,12.8 \mathrm{~Hz}, 0.8 \mathrm{H}$, $\left.\mathrm{NCH}_{\mathrm{A}} \mathbf{H}_{\mathrm{B}} \mathrm{R}\right), 2.42(\mathrm{dd}, 1 \mathrm{H}), 2.36-1.58(\mathrm{~m}, 7 \mathrm{H}), 1.76\left(\mathrm{~s}, 3 \mathrm{H}, \mathrm{CH}_{3}\right), 1.69\left(\mathrm{~s}, 3 \mathrm{H}, \mathrm{CH}_{3}\right)$.

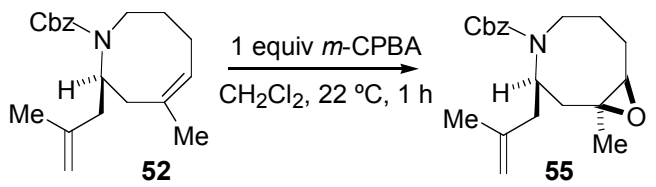

55. A round-bottomed flask was charged with $36.6 \mathrm{mg}(0.117 \mathrm{mmol})$ of $\mathbf{5 2}$ in $1.2 \mathrm{~mL}$ $\mathrm{CH}_{2} \mathrm{Cl}_{2}$. The resulting mixture was cooled to $0{ }^{\circ} \mathrm{C}$ in an ice bath. $m \mathrm{CPBA}, 21.1 \mathrm{mg}(0.123$ mmol, commercial reagent washed with $\mathrm{pH} 7$ buffer and dried in vacuo) was added in one portion to the stirring solution and the reaction was then allowed to warm to ambient temperature. After $1 \mathrm{~h}$, TLC analysis indicated that most of the starting material had been consumed. The reaction was then diluted with $\mathrm{CH}_{2} \mathrm{Cl}_{2}, \sim 5 \mathrm{~mL}$, and transferred to a separatory funnel. The solution was washed with $\sim 5 \mathrm{~mL}$ of a $20 \% \mathrm{wt} / \mathrm{wt}$ aqueous solution of $\mathrm{NaHSO}_{3}$ followed by $\sim 5 \mathrm{~mL}$ of a $10 \% \mathrm{wt} / \mathrm{wt}$ aqueous solution of $\mathrm{NaHCO}_{3}$. The organic phase was separated, dried over $\mathrm{Na}_{2} \mathrm{SO}_{4}$, filtered and concentrated in vacuo to a colorless oil. ${ }^{1} \mathrm{H}$ NMR analysis of the unpurified reaction mixture indicated $\sim 95 \%$ conversion of the starting material. The product was purified by silica gel chromatography with 2:1 hexane:Et ${ }_{2} \mathrm{O}$ as the eluent. Cbz-protected azocine oxirane 55 was thus obtained in $82 \%$ yield $(31.7 \mathrm{mg}, 0.096 \mathrm{mmol})$. IR (neat): $2932(\mathrm{~m}), 1698(\mathrm{~s})$, 1480 (m), 1424 (m), 1338 (m), 1269 (m), 1126 (m), 1058 (w) cm ${ }^{-1} ;{ }^{1} \mathrm{H}$ NMR (400 MHz, $\left.\mathrm{CDCl}_{3}, 25^{\circ} \mathrm{C}\right), 2: 3$ mixture of amide rotamers: $\delta 7.37-7.28(\mathrm{~m}, 5 \mathrm{H}, \mathrm{ArH}), 5.16(\mathrm{ABq}, J=$ $\left.12.5 \mathrm{~Hz}, 1.2 \mathrm{H}, \mathrm{PhCH}_{2} \mathrm{O}\right), 5.11\left(\mathrm{~s}, 0.8 \mathrm{H}, \mathrm{PhCH}_{2} \mathrm{O}\right), 4.79-4.72\left(\mathrm{~m}, 0.6 \mathrm{H}, \mathrm{NCHR}_{2}\right), 4.72$ (s(br), $\left.1 \mathrm{H}, \mathrm{R}_{2} \mathrm{C}=\mathrm{CH}_{\mathrm{A}} \mathrm{H}_{\mathrm{B}}\right), 4.62\left(\mathrm{~d}, J=8.1 \mathrm{~Hz}, 1 \mathrm{H}, \mathrm{R}_{2} \mathrm{C}=\mathrm{CH}_{\mathrm{A}} \mathbf{H}_{\mathbf{B}}\right), 4.47$ (ddd, $J=17.9$, 
9.5, $\left.4.8 \mathrm{~Hz}, 0.4 \mathrm{H}, \mathrm{NCHR}_{2}\right), 3.74$ (d(br), $\left.J=14.3 \mathrm{~Hz}, 0.4 \mathrm{H}, \mathrm{NCH}_{\mathrm{A}} \mathrm{H}_{\mathrm{B}} \mathrm{R}\right), 3.63$ (d (br), $J=$ $\left.15.0 \mathrm{~Hz}, 0.6 \mathrm{H}, \mathrm{NCH}_{\mathrm{A}} \mathrm{H}_{\mathrm{B}} \mathrm{R}\right), 2.74-2.58(\mathrm{~m}, 2 \mathrm{H}), 2.34-1.17(\mathrm{~m}, \sim 8 \mathrm{H}), 1.76\left(\mathrm{~s}, 1.8 \mathrm{H}, \mathrm{CH}_{3}\right)$, $1.61\left(\mathrm{~s}, 1.2 \mathrm{H}, \mathrm{CH}_{3}\right), 1.33\left(\mathrm{~s}, 1.2 \mathrm{H}, \mathrm{CH}_{3}\right), 1.23\left(\mathrm{~s}, 1.2 \mathrm{H}, \mathrm{CH}_{3}\right) ;{ }^{13} \mathrm{C}$ NMR $(100 \mathrm{MHz}$, $\left.\mathrm{CDCl}_{3}\right): \delta 156.6,156.0,142.4,141.7,137.2,136.6,128.6,128.54,128.48,128.3,128.0$, $127.9,113.9,113.4,67.4,67.1,62.4,62.2,59.5,59.2,51.0,50.7,43.7,42.9,42.2$. 42.0, 37.3, 37.0, 30.1, 29.9, 23.8, 22.8, 22.24, 22.21, 22.19, 21.95; HRMS Calcd for $\mathrm{C}_{20} \mathrm{H}_{27} \mathrm{NO}_{3}(\mathrm{M}+\mathrm{Na}): 352.1889$, Found: 352.1879 .

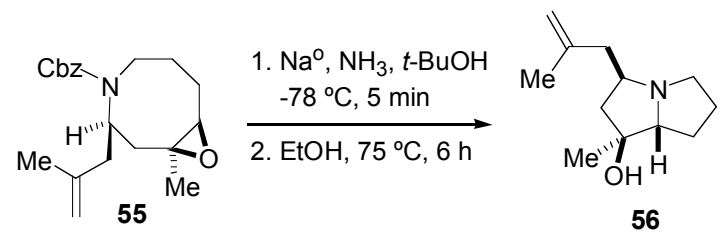

56. Deprotection of 55 was performed according to the procedure for the preparation of 54. The derived unprotected azocine oxirane was obtained in $77 \%$ yield after silica gel chromatography in $30: 1 \quad \mathrm{CH}_{2} \mathrm{Cl}_{2}$ washed with $2 \% \mathrm{v} / \mathrm{v}$ concentrated $\mathrm{NH}_{4} \mathrm{OH}$ (Dragendorff's reagent for TLC visualization). IR (neat): 3364 (w), $3081(\mathrm{w}), 2924(\mathrm{~s})$, 2855 (m), 1646 (w), 1470 (m), 1382 (m), $1143(\mathrm{~m}), 897$ (m) cm ${ }^{-1} ;{ }^{1} \mathrm{H}$ NMR (400 MHz, $\mathrm{CDCl}_{3}$ ): $\delta 4.85$ (s(br), $\left.1 \mathrm{H}, \mathrm{R}_{2} \mathrm{C}=\mathrm{CH}_{\mathrm{A}} \mathrm{H}_{\mathrm{B}}\right), 4.75$ (s(br), 1H, $\left.\mathrm{R}_{2} \mathrm{C}=\mathrm{CH}_{\mathrm{A}} \mathbf{H}_{\mathbf{B}}\right), 3.04$ (ddd, $J=$ 14.6, 3.7, 3.3 Hz, 1H, NCH), 2.91-2.85 (m, 1H, NCH), 2.72 (dd, $J=9.9,3.7 \mathrm{~Hz}, 1 \mathrm{H}$, ROCHR), 2.47 (dddd, $J=11.4,11.4,2.6,2.6 \mathrm{~Hz}, 1 \mathrm{H}, \mathrm{NCH}), 2.16-2.00$ (m, 3H), 1.86 (d, $J=13.9 \mathrm{~Hz}, 1 \mathrm{H}), 1.73\left(\mathrm{~s}, 3 \mathrm{H}, \mathrm{CH}_{3}\right), 1.66-1.43(\mathrm{~m}, 5 \mathrm{H}), 1.38\left(\mathrm{~s}, 3 \mathrm{H}, \mathrm{CH}_{3}\right) ;{ }^{13} \mathrm{C} \mathrm{NMR}$ $\left(100 \mathrm{MHz}, \mathrm{CDCl}_{3}\right): \delta 142.9,113.7,63.4,59.3,54.0,45.5,45.4,42.4,27.2,25.7,25.7$, 22.7, 22.2; HRMS Calcd for $\mathrm{C}_{12} \mathrm{H}_{21} \mathrm{NO}(\mathrm{M}+\mathrm{H})$ : 196.1701, Found: 196.1701. The secondary amine was subjected to transannular ring-closure according to the following method: A 1-dram vial was charged with $8.4 \mathrm{mg}(43 \mu \mathrm{mol})$ of the unprotected azocine oxirane and $0.5 \mathrm{~mL}$ EtOH (distilled from $\mathrm{CaH}_{2}$ ). The vial was sealed and the reaction was stirred for $6 \mathrm{~h}$ at $75{ }^{\circ} \mathrm{C}$. After cooling to ambient temperature, the solution was concentrated in vacuo. ${ }^{1} \mathrm{H}$ NMR analysis of the unpurified mixture indicated $\sim 90 \%$ conversion to the desired bicycle. Subsequent purification through a short plug of silica gel with 4:1 $\mathrm{CH}_{2} \mathrm{Cl}_{2}: \mathrm{MeOH}$ washed with $2 \% \mathrm{v} / \mathrm{v}$ concentrated $\mathrm{NH}_{4} \mathrm{OH}$ as an eluent (Dragendorff's reagent for TLC visualization) delivered $8.1 \mathrm{mg}$ (41 $\mu \mathrm{mol}, 72 \%$ yield) of 56 as a white solid. Crystals sufficient for x-ray analysis were obtained from heptane. See below for data. IR (neat): 3364 (s), 3087 (m), 2968 (s), 1652 (w), 1464 (m), $1388(\mathrm{~m})$, $1281(\mathrm{w}), 1149(\mathrm{~m}), 897(\mathrm{~m}) \mathrm{cm}^{-1} ;{ }^{1} \mathrm{H} \mathrm{NMR}\left(400 \mathrm{MHz}, \mathrm{CDCl}_{3}\right): \delta 4.76$ (s(br), 1H, $\mathrm{R}_{2} \mathrm{C}=\mathrm{CH}_{\mathrm{A}} \mathrm{H}_{\mathrm{B}}$ ), 4.73 (s(br), $\left.1 \mathrm{H}, \mathrm{R}_{2} \mathrm{C}=\mathrm{CH}_{\mathrm{A}} \mathbf{H}_{\mathrm{B}}\right), 2.96$ (ddd, $J=8.4,8.4,5.1 \mathrm{~Hz}, 1 \mathrm{H}$, $\left.\mathrm{NCH}_{\mathrm{A}} \mathrm{H}_{\mathrm{B}} \mathrm{R}\right), \quad 2.79 \quad\left(\mathrm{~s}(\mathrm{br}), \quad 1 \mathrm{H}, \quad \mathrm{NCH}\left(\mathrm{CH}_{2} \mathrm{C}(\mathrm{Me})=\mathrm{CH}_{2}\right) \mathrm{R}\right), \quad 2.62 \quad(\mathrm{~s}(\mathrm{br}), \quad 1 \mathrm{H}$, $\mathrm{NCH}(\mathrm{C}(\mathrm{Me})(\mathrm{R}) \mathrm{OH}) \mathrm{R}), 2.39\left(\mathrm{dd}, J=13.9,5.5 \mathrm{~Hz}, 1 \mathrm{H}, \mathrm{RCH}_{\mathrm{A}} \mathrm{H}_{\mathrm{B}} \mathrm{C}(\mathrm{Me})=\mathrm{CH}_{2}\right), 2.29$ (s(br), 1H, $\left.\mathrm{NCH}_{\mathrm{A}} \mathbf{H}_{\mathrm{B}} \mathrm{R}\right), 2.17$ (dd, $\left.J=13.6,8.1 \mathrm{~Hz}, 1 \mathrm{H}, \mathrm{NCH}(\mathrm{R}) \mathrm{CH}_{\mathrm{A}} \mathrm{H}_{\mathrm{B}} \mathrm{R}\right), 2.09$ (dd, $J=$ 14.6, $\left.8.8 \mathrm{~Hz}, 1 \mathrm{H}, \mathrm{RCH}_{\mathrm{A}} \mathrm{H}_{\mathrm{B}} \mathrm{C}(\mathrm{Me})=\mathrm{CH}_{2}\right), 2.04-1.92(\mathrm{~m}, 1 \mathrm{H}), 1.85(\mathrm{dd}, J=13.9,7.3 \mathrm{~Hz}$, $\left.1 \mathrm{H}, \mathrm{NCH}(\mathrm{R}) \mathrm{CH}_{\mathrm{A}} \mathbf{H}_{\mathrm{B}} \mathrm{R}\right), 1.74\left(\mathrm{~s}, 3 \mathrm{H}, \mathrm{R}_{2} \mathrm{CCH}_{3}\right), 1.68-1.57(\mathrm{~m}, 1 \mathrm{H}), 1.26(\mathrm{~s}, 3 \mathrm{H}$, $\left.\mathrm{R}_{2} \mathrm{C}(\mathrm{OH}) \mathrm{CH}_{3}\right) ;{ }^{13} \mathrm{C} \mathrm{NMR}\left(100 \mathrm{MHz}, \mathrm{CDCl}_{3}\right): \delta 143.6,112.1,77.3,74.8,57.2,50.5,47.0$, 43.1, 27.2, 25.0, 23.0, 20.8; HRMS Calcd for $\mathrm{C}_{12} \mathrm{H}_{21} \mathrm{NO}(\mathrm{M}+\mathrm{H})$ : 196.1701, Found: 196.1704; $[\alpha]_{\mathrm{D}}+25.8\left(c=0.25, \mathrm{CHCl}_{3}\right)$. 


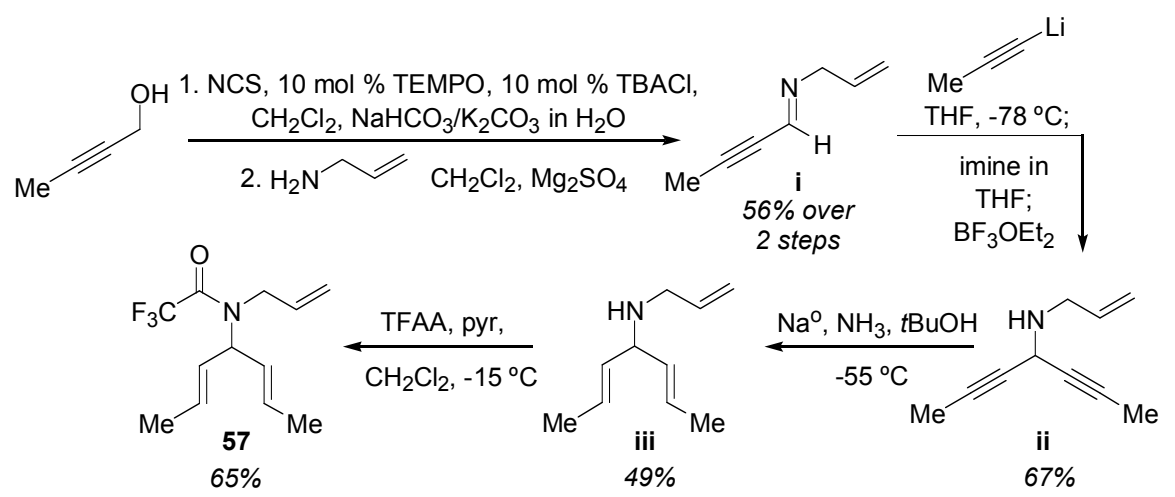

57. Trifluoroacetamide $\mathbf{5 7}$ was prepared according to a modified sequence reported by Blechert and coworkers for a related unsaturated amine. ${ }^{17}$

i. Oxidation of 2-butyn-1-ol was accomplished according to published procedure ${ }^{18}$ with some modification. To a rapidly stirring solution of 2-butyn-1-ol $(1.15 \mathrm{~mL}, 16.0$ mmol) in $32 \mathrm{~mL}$ of $\mathrm{CH}_{2} \mathrm{Cl}_{2}$ and $32 \mathrm{~mL}$ of an aqueous solution of $\mathrm{NaHCO}_{3}(0.5 \mathrm{M})$ and $\mathrm{K}_{2} \mathrm{CO}_{3}(0.05 \mathrm{M})$ was added TBACl $(443 \mathrm{mg}, 1.60 \mathrm{mmol})$ followed by TEMPO $(250 \mathrm{mg}$, $1.60 \mathrm{mmol})$. To the resulting light orange mixture was added NCS $(3.43 \mathrm{~g}, 25.6 \mathrm{mmol}$, recrystallized from toluene) in one portion. The mixture was then stirred for $6 \mathrm{~h}$. At this time, the aqueous layer was separated and washed with two $30 \mathrm{~mL}$ portions of $\mathrm{CH}_{2} \mathrm{Cl}_{2}$. The combined organic fractions were washed with a saturated aqueous solution of $\mathrm{NaCl}$ $(150 \mathrm{~mL})$, dried over $\mathrm{MgSO}_{4}$ and filtered. A small aliquot $(\sim 3 \mathrm{~mL})$ of this solution was carefully concentrated under reduced pressure. Analysis by ${ }^{1} \mathrm{H}$ NMR spectroscopy revealed $>98 \%$ conversion of the alcohol to the desired product. ${ }^{1} \mathrm{H}$ NMR $(400 \mathrm{MHz}$, $\left.\mathrm{CDCl}_{3}\right): \delta 9.15(\mathrm{~s}(\mathrm{br}), 1 \mathrm{H}, \mathrm{RCH}=\mathrm{O}), 2.07$ (s(br), 3H, $\left.\mathbf{C H}_{3}\right)$. The unpurified solution of aldehyde was directly subjected to imine formation: $\mathrm{MgSO}_{4}$ was added followed by 1 equiv. of allyl amine (assuming quantitative yield for the oxidation, $1.20 \mathrm{~mL}, 16.0$ mmol). The resulting mixture was stirred for $6 \mathrm{~h}$, filtered, and concentrated in vacuo. ${ }^{1} \mathrm{H}$ NMR analysis of the unpurified reaction indicated complete consumption of the aldehyde. Kugelrohr distillation of the reaction mixture (high vacuum, $\sim 60-80{ }^{\circ} \mathrm{C}$ ) delivered $0.97 \mathrm{~g}$ of the imine $\mathbf{i}$ as a pale orange oil. ${ }^{1} \mathrm{H}$ NMR $\left(400 \mathrm{MHz}, \mathrm{CDCl}_{3}\right), \sim 1: 1$ mixture of cis and trans isomers: $\delta 7.49-7.46(\mathrm{~m}, 1 \mathrm{H}, \mathrm{N}=\mathrm{CHR}), 6.06-5.91(\mathrm{~m}, 1 \mathrm{H}$, $\left.\mathrm{RCH}=\mathrm{CH}_{2}\right), 5.23-5.10\left(\mathrm{~m}, 2 \mathrm{H}, \mathrm{RCH}=\mathrm{CH}_{2}\right), 4.24(\mathrm{~m}, 1 \mathrm{H}, \mathrm{NCH}), 4.11(\mathrm{~m}, 1 \mathrm{H}, \mathrm{NCH})$, 2.04 (d(br), $\left.J=1.8 \mathrm{~Hz}, 1.5 \mathrm{H}, \mathrm{CH}_{3}\right), 1.99$ (d(br), $J=1.8 \mathrm{~Hz}, 1.5 \mathrm{H}, \mathrm{CH}_{3}$ ).

ii. A modified procedure for the alkylation of imine $\mathbf{i}$ with the requisite propargyl lithium was adapted from the literature. ${ }^{19}$ Propyne $(\sim 2 \mathrm{~mL})$ was condensed into $5 \mathrm{~mL}$ of THF in a graduated 2-neck tube at $-78{ }^{\circ} \mathrm{C}$. The solution was made homogeneous by gentle shaking of the tube. The resulting mixture was then transferred via cannula to a $100 \mathrm{~mL}$ round-bottom flask containing $30 \mathrm{~mL}$ of THF at $-78{ }^{\circ} \mathrm{C}$. A solution of $n \mathrm{BuLi}$ (1.59 $\mathrm{M}$ in hexanes, $8.50 \mathrm{~mL}, 13.5 \mathrm{mmol}$ ) was then added. The resulting milky solution was stirred at $-78{ }^{\circ} \mathrm{C}$ for $30 \mathrm{~min}$. A solution of imine $\mathbf{i}(725 \mathrm{mg}, 6.77 \mathrm{mmol})$ in $\sim 3 \mathrm{~mL}$ of THF was added via cannula ( $\sim 2 \mathrm{~mL}$ of THF to complete the transfer). Subsequently, $\mathrm{BF}_{3} \mathrm{OEt}_{2}\left(1.71 \mathrm{~mL}, 13.5 \mathrm{mmol}\right.$, distilled from $\left.\mathrm{CaH}_{2}\right)$ was added dropwise via syringe.

(17) C. M. Huew, S. Blechert Synthesis 1997, 61-67.

(18) J. Einhorn, C. Einhorn, F. Ratajczak, J-L. Pierre J. Org. Chem. 1996, 61, 7452-7454.

(19) Aubrecht, K. B.; Winemiller, M. D.; Collum, D. B. J. Am. Chem. Soc. 2000, 122, 11084-11089. 
The resulting mixture, while stirring for an additional $30 \mathrm{~min}$ at $-78{ }^{\circ} \mathrm{C}$, turned pale yellow. It was then allowed to warm to ambient temperature ( $\sim 30 \mathrm{~min}) . \sim 10 \mathrm{~mL}$ of a 10\% wt/wt aqueous solution of $\mathrm{NaOH}$ was then added and the resulting mixture was stirred rapidly for $\sim 15 \mathrm{~min}$. The now orange solution was poured into a separatory funnel and diluted with $\mathrm{H}_{2} \mathrm{O}$. The aqueous phase was washed $3 \mathrm{x}$ with $30 \mathrm{~mL}$ portions of $\mathrm{Et}_{2} \mathrm{O}$. The combined organic fractions were washed with a saturated aqueous solution of $\mathrm{NaCl}$, dried over $\mathrm{Na}_{2} \mathrm{SO}_{4}$, filtered and concentrated. Purification with silica gel chromatography $(30: 1$ $\mathrm{CH}_{2} \mathrm{Cl}_{2}$ washed with $2 \% \mathrm{v} / \mathrm{v}$ concentrated $\left.\mathrm{NH}_{4} \mathrm{OH}\right)$ gave $571 \mathrm{mg}(3.87 \mathrm{mmol})$ of the desired amine ii in $67 \%$ yield. ${ }^{1} \mathrm{H}$ NMR (400 $\left.\mathrm{MHz}, \mathrm{CDCl}_{3}\right): \delta 5.91$ (ddt, $J=16.1,10.3$, $\left.5.9 \mathrm{~Hz}, 1 \mathrm{H}, \mathrm{RCH}=\mathrm{CH}_{2}\right), 5.23\left(\mathrm{ddd}, J=17.2,3.3,1.5 \mathrm{~Hz},{ }^{1} \mathrm{H}, \mathrm{RCH}=\mathrm{CH}_{\mathrm{A}} \mathrm{H}_{\mathrm{B}}\right), 5.11$ (ddd, $\left.J=10.3,2.9,1.1 \mathrm{~Hz}, 1 \mathrm{H}, \mathrm{RCH}=\mathrm{CH}_{\mathrm{A}} \mathbf{H}_{\mathbf{B}}\right), 4.27(\mathrm{p}, J=2.2 \mathrm{~Hz}, 1 \mathrm{H}, \mathrm{NCHR} 2), 3.36$ (dt, $J=$ 5.9, $\left.1.5 \mathrm{~Hz}, 2 \mathrm{H}, \mathrm{NCH}_{2} \mathrm{R}\right), 1.85$ (d, $\left.J=2.2 \mathrm{~Hz}, 6 \mathrm{H}, \mathrm{CH}_{3}\right), 1.43$ (s(br), 1H, NH).

iii. Metallic $\mathrm{Na}(\sim 140 \mathrm{mg}, 8.1 \mathrm{mmol}, 6$ equiv., washed with hexanes) was placed in a $50 \mathrm{~mL}$ two-necked flask containing a teflon stirbar. The flask was then fitted with a Dewar condenser and purged with $\mathrm{N}_{2}$. After cooling the flask and condenser to $-78{ }^{\circ} \mathrm{C}$, $\sim 45 \mathrm{~mL}$ of $\mathrm{NH}_{3}(0.03 \mathrm{M}$ with respect to amine ii) was condensed onto the metallic $\mathrm{Na}$ with stirring to produce a deep blue solution. A solution of amine ii (198 mg, $1.34 \mathrm{mmol}$ )

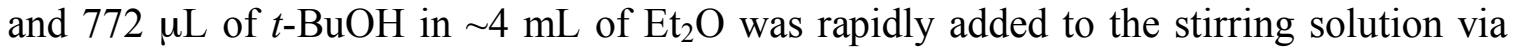
cannula. An additional $1 \mathrm{~mL}$ of $\mathrm{Et}_{2} \mathrm{O}$ was used for complete transfer. The resulting mixture should remain deep blue. If the color dissipates to white or pale yellow, a second portion of metallic $\mathrm{Na}$ was added. The reaction was stirred for $45 \mathrm{~min}$ at $-55^{\circ} \mathrm{C}$. At this time, the blue color disappears (or solid $\mathrm{NH}_{4} \mathrm{Cl}$ was added in small portions until the blue color dissipates). The mixture was diluted with $\mathrm{CH}_{2} \mathrm{Cl}_{2}(\sim 15 \mathrm{~mL})$ and then allowed to gradually warm to ambient temp (without stirring). After bubbling (of $\mathrm{NH}_{3}$ ) had ceased, the mixture was transferred into a separatory funnel with $\sim 5 \mathrm{~mL} \mathrm{CH}_{2} \mathrm{Cl}_{2}$ and $\sim 20 \mathrm{~mL}$ $\mathrm{H}_{2} \mathrm{O}$. The aqueous layer was washed $3 \mathrm{x}$ with $20 \mathrm{~mL}$ portions of $\mathrm{CH}_{2} \mathrm{Cl}_{2}$. The combined organic extracts were dried over $\mathrm{Na}_{2} \mathrm{SO}_{4}$, filtered and concentrated under reduced pressure to give an orange oil. ${ }^{1} \mathrm{H}$ NMR analysis indicated that the unpurified product was contaminated with $\sim 20 \%$ of the over-reduced propyl amine. Purification by silica gel chromatography $\left(4: 1 \mathrm{CH}_{2} \mathrm{Cl}_{2}: \mathrm{Et}_{2} \mathrm{O}\right.$ washed with $2 \% \mathrm{v} / \mathrm{v}$ concentrated $\mathrm{NH}_{4} \mathrm{OH}$, Dragendorff's reagent for TLC visualization) gave $99.4 \mathrm{mg}(0.657 \mathrm{mmol})$ of the desired unsaturated amine iii (50\% yield). ${ }^{1} \mathrm{H}$ NMR (400 $\left.\mathrm{MHz} \mathrm{CDCl}_{3}\right): \delta 5.90$ (ddt, $J=16.1$, 10.3, $\left.5.9 \mathrm{~Hz}, 1 \mathrm{H}, \mathrm{RCH}=\mathrm{CH}_{2}\right), 5.57$ (ddd, $\left.J=15.0,12.8,6.2 \mathrm{~Hz}, 2 \mathrm{H}, \mathrm{RCH}=\mathrm{CHR}\right), 5.37$ (ddq, $J=15.4,7.7,1.5 \mathrm{~Hz}, 2 \mathrm{H}, \mathrm{RCH}=\mathrm{CHR}), 5.14$ (ddd, $J=17.2,3.3,1.5 \mathrm{~Hz}, 1 \mathrm{H}$, $\left.\mathrm{RCH}=\mathrm{CH}_{\mathrm{A}} \mathrm{H}_{\mathrm{B}}\right), 5.06\left(\mathrm{ddd}, J=10.3,2.9,1.1 \mathrm{~Hz}, 1 \mathrm{H}, \mathrm{RCH}=\mathrm{CH}_{\mathrm{A}} \mathbf{H}_{\mathbf{B}}\right), 3.54(\mathrm{t}, J=7.7 \mathrm{~Hz}$, $1 \mathrm{H}, \mathrm{NCHR}_{2}$ ), 3.18 (dt, $\left.J=5.9,1.5 \mathrm{~Hz}, 2 \mathrm{H}, \mathrm{NCH}_{2} \mathrm{R}\right), 1.68$ (dd, $J=6.6,1.5 \mathrm{~Hz}, 6 \mathrm{H}, \mathrm{CH}_{3}$ ), 1.13 (s(br), 1H, NH).

57. To a $-15{ }^{\circ} \mathrm{C} \quad 0.1 \mathrm{M}$ solution of amine iii $(97 \mathrm{mg}, 0.64 \mathrm{mmol})$ in pyridine: $\mathrm{CH}_{2} \mathrm{Cl}_{2}(1: 3 \mathrm{v} / \mathrm{v})$ was slowly added trifluoroacetic anhydride $(0.18 \mathrm{~mL}, 1.3$ mmol) dropwise. The reaction was stirred at ambient temperature for $2 \mathrm{~h}$, at which time TLC analysis indicated that no starting material remained. The solution was then concentrated in vacuo, and the resulting oil was redissolved in $\mathrm{CH}_{2} \mathrm{Cl}_{2}(\sim 2 \mathrm{~mL})$. Silica gel was then added until a thick slurry formed. A purge of $\mathrm{N}_{2}$, followed by reduced pressure lead to the removal of solvent. The mixture, now absorbed onto silica gel, was transferred to the top of a silica gel column packed with 15:1 hexanes:EtOAc. The desired product, TFA-protected amine 57 was obtained in $65 \%$ yield $(101 \mathrm{mg}, 0.41 \mathrm{mmol})$ after eluting 
with 15:1 hexanes:EtOAc (anisaldehyde stain for TLC visualization). ${ }^{1} \mathrm{H}$ NMR (400 $\left.\mathrm{MHz}, \mathrm{CDCl}_{3}, 25 \mathrm{C}\right), \sim 1: 1$ mixture of amide rotamers: $\delta$ 5.83-5.72 (m, $\left.1 \mathrm{H}, \mathrm{RCH}=\mathrm{CH}_{2}\right)$, 5.71-5.58 (m, 3H, RCH=CHR), 5.49 (dd, $J=15.4,5.5 \mathrm{~Hz}, 1 \mathrm{H}, \mathrm{RCH}=\mathrm{CHR}), 5.22-5.11$ $\left(\mathrm{m}, 2 \mathrm{H}, \mathrm{RCH}=\mathrm{CH}_{2}\right), 5.00-4.95\left(\mathrm{~m}, 1 \mathrm{H}, \mathrm{NCHR}_{2}\right), 3.99\left(\mathrm{~d}, J=5.1 \mathrm{~Hz}, 1 \mathrm{H}, \mathrm{NCH}_{2} \mathrm{R}\right), 3.89$ (d, $\left.J=5.5 \mathrm{~Hz}, 1 \mathrm{H}, \mathrm{NCH}_{2} \mathrm{R}\right), 1.74-1.70\left(\mathrm{~m}, 3 \mathrm{H}, \mathrm{CH}_{3}\right)$; HRMS Calcd for $\mathrm{C}_{12} \mathrm{H}_{16} \mathrm{~F}_{3} \mathrm{NO}$ $(\mathrm{M}+\mathrm{Na}): 270.1082$, Found: 270.1082.

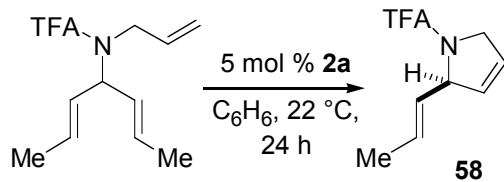

58. General procedure for ARCM was followed. Silica gel chromatography with 9:1 hexane: $\mathrm{Et}_{2} \mathrm{O}$ was used for purification ( $70 \%$ yield, $\left.0.045 \mathrm{mmol}\right)$. IR (neat): 2924 (w), 2879 (w), 1714 (s), 1463 (m), 1230 (s), 1213 (s), 1146 (s), 962 (w) cm ${ }^{-1}$; ${ }^{1}$ H NMR (400 $\mathrm{MHz}, \mathrm{CDCl}_{3}, 25^{\circ} \mathrm{C}$ ), $\sim 1: 4$ mixture of amide rotamers: $\delta 5.87-5.64(\mathrm{~m}, 3 \mathrm{H}), 5.41$ (dddd, $J$ $=15.4,7.3,3.3,1.5 \mathrm{~Hz}, 0.8 \mathrm{H}), 5.37-5.30(\mathrm{~m}, 0.2 \mathrm{H}), 5.25\left(\mathrm{~s}(\mathrm{br}), 1 \mathrm{H}, \mathrm{NCHR}_{2}\right), 4.43(\mathrm{ABq}$, $\left.J=15.0 \mathrm{~Hz}, 1.6 \mathrm{H}, \mathrm{NCH}_{2} \mathrm{R}\right), 4.34\left(\mathrm{ABq}, J=17.6 \mathrm{~Hz}, 0.4 \mathrm{H}, \mathrm{NCH}_{2} \mathrm{R}\right), 1.70$ (dd, $J=6.6$, $\left.1.8 \mathrm{~Hz}, 3 \mathrm{H}, \mathrm{CH}_{3}\right), 5.49$ (dd, $\left.J=15.4,5.5 \mathrm{~Hz}, 1 \mathrm{H}, \mathrm{RCH}=\mathrm{CHR}\right), 5.22-5.11(\mathrm{~m}, 2 \mathrm{H}$, $\left.\mathrm{RCH}=\mathrm{CH}_{2}\right), 5.00-4.95\left(\mathrm{~m}, 1 \mathrm{H}, \mathrm{NCHR}_{2}\right), 3.99\left(\mathrm{~d}, J=5.1 \mathrm{~Hz}, 1 \mathrm{H}, \mathrm{NCH}_{2} \mathrm{R}\right), 3.89(\mathrm{~d}, J=$ $\left.5.5 \mathrm{~Hz}, 1 \mathrm{H}, \mathrm{NCH}_{2} \mathrm{R}\right), 1.74-1.70\left(\mathrm{~m}, 3 \mathrm{H}, \mathrm{CH}_{3}\right) ;{ }^{13} \mathrm{C} \mathrm{NMR}\left(100 \mathrm{MHz}, \mathrm{CDCl}_{3}\right): \delta 155.3(\mathrm{q}$, $J=36.4 \mathrm{~Hz}), 130.0,129.8,129.2,128.94,128.91,128.6,127.3,124.13,124.11,123.6$, $116.3(\mathrm{q}, J=287 \mathrm{~Hz}), 67.3,66.45,66.42,54.8,52.9$ (q, $J=3.8 \mathrm{~Hz}), 31.7,28.1,22.8$, 22.6, 17.8, 17.7, 14.2; HRMS Calcd for $\mathrm{C}_{9} \mathrm{H}_{10} \mathrm{~F}_{3} \mathrm{NO}(\mathrm{M}+\mathrm{Na})$ : 228.0612, Found: $228.0608 ;[\alpha]_{\mathrm{D}}-86.8\left(c=0.5, \mathrm{CHCl}_{3}\right)$.

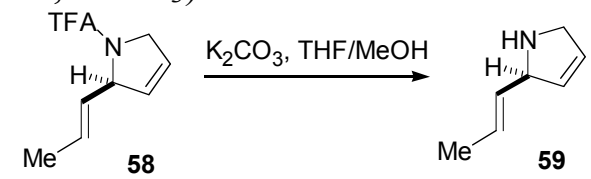

59. Trifluoroacetamide 58 (13.6 mg, $0.066 \mathrm{mmol})$ was combined with $2 \mathrm{~mL}$ of a $\mathrm{K}_{2} \mathrm{CO}_{3}$ solution $\left(10 \%\right.$ in 5:2 MeOH: $\left.\mathrm{H}_{2} \mathrm{O}\right)$. The mixture was allowed to stir for $15 \mathrm{~h}$ and then transferred to a separatory funnel with $\mathrm{CH}_{2} \mathrm{Cl}_{2}$ and $\mathrm{H}_{2} \mathrm{O}$. The aqueous layer was washed three times with $2 \mathrm{~mL}$ portions of $\mathrm{CH}_{2} \mathrm{Cl}_{2}$. The combined organic fractions were dried over $\mathrm{Na}_{2} \mathrm{SO}_{4}$, filtered, and carefully concentrated with a stream of $\mathrm{N}_{2}$ to give amine $\mathbf{5 9}$ (3.7 mg, $0.032 \mathrm{mmol}, 50 \%$ yield) as an oil. ${ }^{1} \mathrm{H}$ NMR (400 MHz, $\left.\mathrm{CDCl}_{3}\right): \delta 5.87(\mathrm{~d}(\mathrm{br}), J$ $=4.8 \mathrm{~Hz}, 1 \mathrm{H}, \mathrm{RCH}=\mathrm{CHR}), 5.71(\mathrm{~s}(\mathrm{br}), 1 \mathrm{H}, \mathrm{RCH}=\mathrm{CHR}), 5.60(\mathrm{ddd}, J=14.3,12.8,6.2$ $\mathrm{Hz}, 1 \mathrm{H}, \mathrm{RCH}=\mathrm{CHR}), 5.40$ (ddd, $J=15.0,7.7,1.5 \mathrm{~Hz}, 1 \mathrm{H}, \mathrm{RCH}=\mathrm{CHR}), 4.43(\mathrm{~s}(\mathrm{br}), 1 \mathrm{H}$, $\mathrm{NCH}), 3.81(\mathrm{~s}(\mathrm{br}), 1 \mathrm{H}, \mathrm{NCH}), 3.73(\mathrm{~s}(\mathrm{br}), 1 \mathrm{H}, \mathrm{NCH}), 1.77(\mathrm{~s}(\mathrm{br}), 1 \mathrm{H}, \mathrm{NH}), 1.68(\mathrm{dd}, J=$ $\left.6.2,1.1 \mathrm{~Hz}, 3 \mathrm{H}, \mathrm{RCH}_{3}\right)$. 


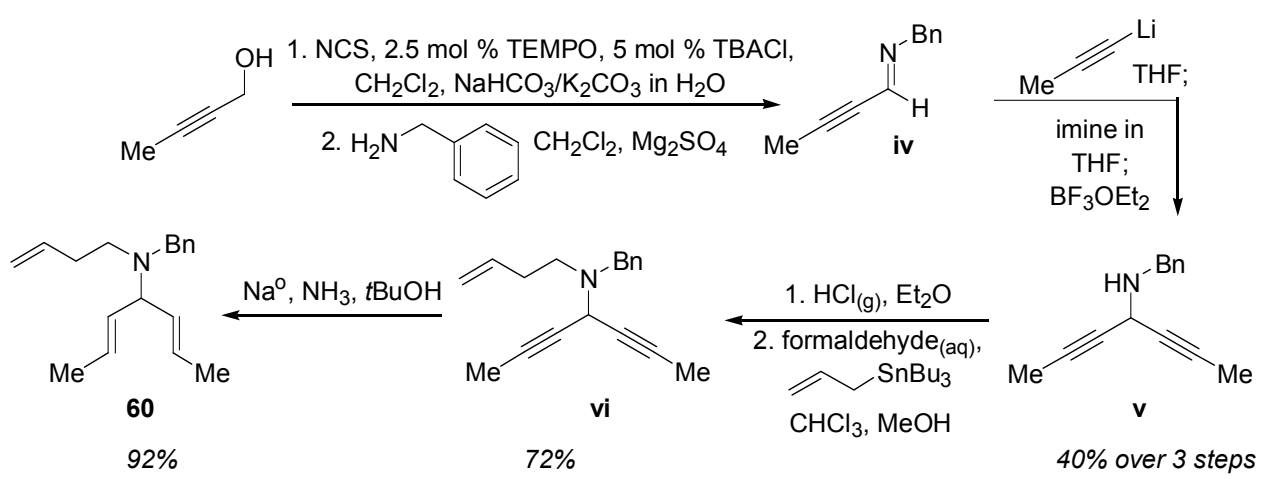

60. The synthesis of $\mathbf{6 0}$ was accomplished in a sequence related to that for the preparation of 57.

iv. The procedure for the preparation of $\mathbf{i}$ was followed (see above) with the following modifications: for the oxidation of 2-butyn-1-ol, $2.5 \mathrm{~mol} \%$ of TEMPO and 5 mol \% TBACl was used. ${ }^{1} \mathrm{H}$ NMR $\left(400 \mathrm{MHz}, \mathrm{CDCl}_{3}\right), \sim 1: 1$ mixture of cis and trans isomers: $\delta 7.55(\mathrm{~s}(\mathrm{br}), 1 \mathrm{H}, \mathrm{N}=\mathrm{CHR}), 7.33-7.21(\mathrm{~m}, 5 \mathrm{H}, \mathrm{ArH}), 4.81\left(\mathrm{~s}(\mathrm{br}), 1 \mathrm{H}, \mathrm{NCH}_{2} \mathrm{Ph}\right)$, 4.66 ( s(br), 1H, $\left.\mathrm{NCH}_{2} \mathrm{Ph}\right), 2.07$ (s(br), 1.5H, $\left.\mathrm{CH}_{3}\right), 1.99$ (s(br), 1.5H, $\mathrm{CH}_{3}$ ).

v. The procedure for the preparation of ii was followed (see above). Imine iv was directly subjected to alkylation without purification. Benzyl amine $\mathbf{v}$ was obtained in $40 \%$ yield over three steps after purification with silica gel chromatography $\left(2: 1\right.$ hexane:Et ${ }_{2} \mathrm{O}$ washed with $2 \% \mathrm{v} / \mathrm{v}$ concentrated $\mathrm{NH}_{4} \mathrm{OH}$ as an eluent). ${ }^{1} \mathrm{H} \mathrm{NMR}\left(400 \mathrm{MHz}, \mathrm{CDCl}_{3}\right)$ : $\delta$ 7.37-7.21 (m, 5H, ArH), $4.24\left(\mathrm{dq}, J=4.4,2.2 \mathrm{~Hz}, 1 \mathrm{H}, \mathrm{NCHR}_{2}\right), 3.90(\mathrm{~s}(\mathrm{br}), 2 \mathrm{H}$, $\left.\mathrm{PhCH}_{2}\right), 1.85$ (t(br), $\left.J=2.2 \mathrm{~Hz}, 6 \mathrm{H}, \mathrm{CH}_{3}\right)$.

vi. The $\mathrm{HCl}$ salt of $\mathbf{v}$ was prepared as follows: through a solution of $\mathbf{v}(2.0 \mathrm{~g})$ in $\sim 50 \mathrm{~mL}$ dry $\mathrm{Et}_{2} \mathrm{O}$ was bubbled $\mathrm{HCl}$ gas for $\sim 1$ min. A gooey solid immediately precipitated. The solvent was then removed in vacuo to give $2.07 \mathrm{~g}$ of an orange foam (88\% yield). ${ }^{1} \mathrm{H}$ NMR (400 MHz, $\left.\mathrm{CDCl}_{3}\right): \delta 7.52-25$ (m, 5H, ArH), $4.43(\mathrm{~s}(\mathrm{br}), 1 \mathrm{H}$, $\left.\mathrm{NCHR}_{2}\right), 4.07$ (s, 2H, PhCH $), 1.85$ (s(br), 6H, $\mathrm{CH}_{3}$ ). This material was then transferred to a round-bottom flask and diluted with 1:1 mixture of $\mathrm{MeOH}: \mathrm{CHCl}_{3}$ to make a $0.5 \mathrm{M}$ solution. A $37 \%$ aqueous solution of formaldehyde $(0.73 \mathrm{~mL})$ was added, followed by $2.86 \mathrm{~mL}$ of allyl tributyl stannane. The reaction was sealed and stirred for $14 \mathrm{~h}$. The mixture was then poured into a separatory funnel and diluted with $\sim 20 \mathrm{~mL}$ of a $1 \mathrm{M}$ aqueous $\mathrm{HCl}$ solution. The organic layer was separated, washed with a saturated aqueous solution of $\mathrm{NaHCO}_{3}$, dried with $\mathrm{Na}_{2} \mathrm{SO}_{4}$, filtered and concentrated. Silica gel chromatography (15:1 hexanes: $\left.\mathrm{Et}_{2} \mathrm{O}\right)$ gave $1.58 \mathrm{~g}$ of vi as a clear oil ( $72 \%$ yield). Note: for more polar amines, the workup described in the literature ${ }^{20}$ is convenient for the facile removal of the stannane byproducts (before chromatography). In this case, unexpectedly, the $\mathrm{HCl}$ salt of $\mathbf{v}$ is not water soluble. vi: ${ }^{1} \mathrm{H} \mathrm{NMR}\left(400 \mathrm{MHz}, \mathrm{CDCl}_{3}\right): \delta 7.37-7.20(\mathrm{~m}$, $5 \mathrm{H}, \mathrm{ArH}), 5.80$ (ddt, $\left.J=16.8,9.9,6.6,6.6 \mathrm{~Hz}, 1 \mathrm{H}, \mathrm{RCH}=\mathrm{CH}_{2}\right), 5.03(\mathrm{~d}(\mathrm{br}), J=17.2 \mathrm{~Hz}$, $\left.1 \mathrm{H}, \mathrm{RCH}=\mathrm{CH}_{\mathrm{A}} \mathrm{H}_{\mathrm{B}}\right), 4.97\left(\mathrm{~d}(\mathrm{br}), J=10.3 \mathrm{~Hz}, 1 \mathrm{H}, \mathrm{RCH}=\mathrm{CH}_{\mathrm{A}} \mathbf{H}_{\mathbf{B}}\right), 4.32(\mathrm{p}, J=2.2 \mathrm{~Hz}$, $\left.1 \mathrm{H}, \mathrm{NCHR}_{2}\right), 3.67$ (s, 2H, PhCH $), 2.66$ (t, $\left.J=7.32 \mathrm{~Hz}, 2 \mathrm{H}, \mathrm{NCH}_{2} \mathrm{R}\right), 2.25$ (dd, $J=14.6$, $\left.6.6 \mathrm{~Hz}, 2 \mathrm{H}, \mathrm{NCH}_{2} \mathrm{CH}_{2} \mathrm{R}\right), 1.85\left(\mathrm{~d}, J=2.2 \mathrm{~Hz}, 6 \mathrm{H}, \mathrm{CH}_{3}\right)$.

60. Into a two-neck round-bottomed flask, equipped with a Dewar condenser (at $78{ }^{\circ} \mathrm{C}$ ), was condensed $\sim 50 \mathrm{~mL}$ of $\mathrm{NH}_{3}$ at $-60{ }^{\circ} \mathrm{C}$. Through the side arm, $555 \mathrm{mg}$ of

(20) Grieco, P. A.; Bahsas. A. J. Org. Chem. 1987, 52, 1378-1380. 
metallic $\mathrm{Na}(32.6 \mathrm{mmol})$ was added in one portion. To the stirring deep blue solution was then added a solution of amine vi $(410 \mathrm{mg}, 1.63 \mathrm{mmol}$, in two portions of $2 \mathrm{~mL} \mathrm{THF}$ ) via cannula. The blue color did not dissipate as the reaction was stirred for $1 \mathrm{~h}$ at $-60{ }^{\circ} \mathrm{C}$. At this time, solid $\mathrm{NH}_{4} \mathrm{Cl}$ was added until the mixture became colorless. $\mathrm{Et}_{2} \mathrm{O}, \sim 5 \mathrm{~mL}$, was added, and the mixture was allowed to warm to ambient temperature. The solution was poured into a separatory funnel and diluted with $\sim 10 \mathrm{~mL} \mathrm{Et}_{2} \mathrm{O}$ and $15 \mathrm{~mL} \mathrm{H}_{2} \mathrm{O}$. The aqueous layer was washed two times with $15 \mathrm{~mL}$ portions of $\mathrm{Et}_{2} \mathrm{O}$. The combined organic fractions were washed with two $30 \mathrm{~mL}$ portions of a saturated aqueous solution of $\mathrm{NaCl}$, dried over $\mathrm{Na}_{2} \mathrm{SO}_{4}$, filtered and concentrated in vacuo. The desired product, 60, was obtained in $92 \%$ yield $(381 \mathrm{mg})$ after silica gel chromatography (30:1 hexanes:EtOAc washed with $2 \% \mathrm{v} / \mathrm{v}$ concentrated $\mathrm{NH}_{4} \mathrm{OH}$, Dragendorff's reagent for TLC visualization). ${ }^{1} \mathrm{H}$ NMR (400 MHz, $\mathrm{CDCl}_{3}$ ): $\delta$ 7.35-7.19 (m, 5H, ArH), $5.76(\mathrm{ddt}, J=17.2,10.3,7.0 \mathrm{~Hz}$, $\left.1 \mathrm{H}, \mathrm{RCH}=\mathrm{CH}_{2}\right), 5.54-5.43(\mathrm{~m}, 2 \mathrm{H}, \mathrm{RCH}=\mathrm{CHR}), 5.48(\mathrm{dd}, J=5.1,5.1 \mathrm{~Hz}, 2 \mathrm{H}$, $\mathrm{RCH}=\mathrm{CHR}), 4.99-4.91\left(\mathrm{~m}, 2 \mathrm{H}, \mathrm{RCH}=\mathrm{CH}_{2}\right), 3.57-3.55(\mathrm{~m}, 1 \mathrm{H}, \mathrm{NCHR}), 3.58(\mathrm{~s}, 2 \mathrm{H}$, $\left.\mathrm{PhCH}_{2}\right), 2.50\left(\mathrm{t}, J=7.5 \mathrm{~Hz}, 2 \mathrm{H}, \mathrm{NCH}_{2} \mathrm{R}\right), 2.15(\mathrm{dd}(\mathrm{br}), J=13.9,7.0 \mathrm{~Hz}, 2 \mathrm{H}$, $\left.\mathrm{NCH}_{2} \mathrm{CH}_{2} \mathrm{R}\right), 1.7\left(\mathrm{dd}, J=4.8,0.7 \mathrm{~Hz}, 6 \mathrm{H}, \mathrm{CH}_{3}\right) ;{ }^{13} \mathrm{C} \mathrm{NMR}\left(100 \mathrm{MHz}, \mathrm{CDCl}_{3}\right): \delta 141.2$, 137.5, 131.5, 128.7, 128.2, 127.7, 126.6, 115.2, 64.2, 54.5, 49.6, 32.6, 18.1; HRMS Calcd for $\mathrm{C}_{18} \mathrm{H}_{25} \mathrm{~N}(\mathrm{M}+\mathrm{H}): 256.2065$, Found: 256.2065.
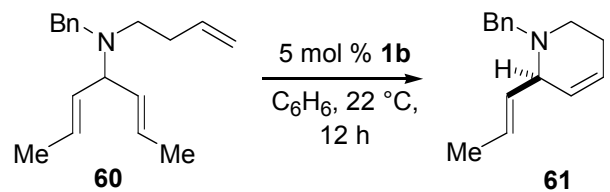

61. General procedure for ARCM was followed. ${ }^{1} \mathrm{H}$ NMR analysis of the unpurified reaction mixture indicated complete conversion of the starting material to the desired product $(90 \%)$ and $\sim 10 \%$ conversion to unidentified byproducts. Silica gel chromatography with 15:1 hexane:EtOAc was used for purification (83\% yield, 0.174 mmol). ${ }^{1} \mathrm{H}$ NMR (400 MHz, $\mathrm{CDCl}_{3}$ ): $\delta$ 7.34-7.19 (m, 5H, ArH), 5.75 (dddd, $J=9.9,4.8$, 2.4, $2.4 \mathrm{~Hz}, 1 \mathrm{H}, \mathrm{RCH}=\mathrm{CHR}), 5.62$ (ddd, $J=15.7,12.8,7.0 \mathrm{~Hz}, 1 \mathrm{H}, \mathrm{RCH}=\mathrm{CHR}), 5.49-$ $5.39(\mathrm{~m}, 2 \mathrm{H}, \mathrm{RCH}=\mathrm{CHR}), 3.98\left(\mathrm{~d}, J=13.6 \mathrm{~Hz}, 1 \mathrm{H}, \mathrm{PhCH}_{\mathrm{A}} \mathrm{H}_{\mathrm{B}}\right), 3.37$ (ddd, $J=8.4,2.6$, $\left.2.6 \mathrm{~Hz}, 1 \mathrm{H}, \mathrm{NCHR}_{2}\right), 3.24\left(\mathrm{~d}, J=13.6 \mathrm{~Hz}, 1 \mathrm{H}, \mathrm{PhCH}_{\mathrm{A}} \mathbf{H}_{\mathrm{B}}\right), 2.81$ (ddd, $J=11.4,4.4,4.4$ $\left.\mathrm{Hz}, 1 \mathrm{H}, \mathrm{NCH}_{\mathrm{A}} \mathrm{H}_{\mathrm{B}} \mathrm{R}\right), 2.25\left(\mathrm{ddd}, J=11.4,8.4,4.4 \mathrm{~Hz}, 1 \mathrm{H}, \mathrm{NCH}_{\mathrm{A}} \mathbf{H}_{\mathrm{B}} \mathrm{R}\right), 2.18-2.08(\mathrm{~m}, 1 \mathrm{H}$, $\mathrm{NCH}_{2} \mathrm{CH}_{\mathrm{A}} \mathrm{H}_{\mathrm{B}} \mathrm{R}$ ), 2.02-1.93 (m, $1 \mathrm{H}, \mathrm{NCH}_{2} \mathrm{CH}_{\mathrm{A}} \mathbf{H}_{\mathrm{B}} \mathrm{R}$ ), 1.71 (dd, $J=6.2,1.5 \mathrm{~Hz}, 3 \mathrm{H}, \mathrm{CH}_{3}$ ); ${ }^{13} \mathrm{C} \mathrm{NMR}\left(100 \mathrm{MHz}, \mathrm{CDCl}_{3}\right): \delta 139.7,132.8,129.8,129.1,128.26,128.23,126.8,125.3$, 63.4, 58.8, 46.7, 26.0, 18.0; Anal. Calcd. for $\mathrm{C}_{15} \mathrm{H}_{19} \mathrm{~N}$ : C, 84.46; H, 8.98; Found: $\mathrm{C}$, 84.59; H, 9.27; $[\alpha]_{\mathrm{D}}-180.9\left(c=1, \mathrm{CHCl}_{3}\right)$.

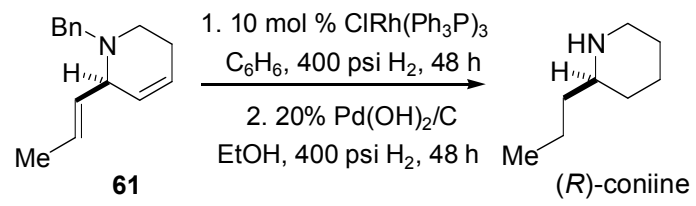

$(\boldsymbol{R})$-Coniine. A test tube containing a teflon stirbar was charged with a solution of amine 61 (105 mg, $0.491 \mathrm{mmol}$ ) in $2.46 \mathrm{~mL} \mathrm{C}_{6} \mathrm{H}_{6}$ and Wilkinson's catalyst (45.4 mg, 0.049 mmol, $10 \mathrm{~mol} \%$, prepared according to literature procedure ${ }^{21}$ ) under an inert atmosphere.

(21) Osborn, J. A.; Wilkinson, G. Inorg. Synth. 1990, 28, 77. 
The container was then placed inside high pressure apparatus, which was subsequently sealed and pressurized to $400 \mathrm{psi}_{2}$. After stirring for $48 \mathrm{~h}$, the reaction vessel was removed from the bomb and the solution was concentrated in vacuo. Analysis by ${ }^{1} \mathrm{H}$ NMR indicated that the starting material had been consumed. Benzyl protected coniine was purified by silica gel chromatography in 10:1 hexane:EtOAc washed with $2 \% \mathrm{v} / \mathrm{v}$ concentrated $\mathrm{NH}_{4} \mathrm{OH}\left(90.9 \mathrm{mg}, 0.419 \mathrm{mmol}, 85 \%\right.$ yield of a pale yellow oil). ${ }^{1} \mathrm{H}$ NMR (400 MHz, $\mathrm{CDCl}_{3}$ ): $\delta$ 7.34-7.20 (m, 5H, ArH), 3.97 (d, $J=13.6 \mathrm{~Hz}, 1 \mathrm{H}, \mathrm{PhCH}_{\mathbf{A}} \mathrm{H}_{\mathrm{B}}$ ), 3.22 (d, $\left.J=13.6 \mathrm{~Hz}, 1 \mathrm{H}, \mathrm{PhCH}_{\mathrm{A}} \mathbf{H}_{\mathbf{B}}\right), 2.73$ (ddd, $\left.J=11.7,3.7,3.7 \mathrm{~Hz}, 1 \mathrm{H}, \mathrm{NCH}\right), 2.31-2.25$ (m, 1H, NCH), 2.05-1.99 (m, 1H, NCH), 1.69-1.26 (m, 10H), 0.91 (dd, $J=7.3,7.3 \mathrm{~Hz}$, $\left.3 \mathrm{H}, \mathrm{CH}_{3}\right) ;{ }^{13} \mathrm{C} \mathrm{NMR}\left(100 \mathrm{MHz}, \mathrm{CDCl}_{3}\right): \delta 140.2,129.1,128.2,126.7,60.8,57.7,51.8$, 34.3, 30.4, 25.3, 23.9, 18.9, 24.8; HRMS Calcd for $\mathrm{C}_{15} \mathrm{H}_{23} \mathrm{~N}(\mathrm{M}+\mathrm{H}): 218.1909$, Found: $218.1905 ;[\alpha]_{\mathrm{D}}-65.7\left(c=1, \mathrm{CHCl}_{3}\right)$. Benzyl deprotection was accomplished through hydrogenolysis as follows: A test-tube equipped with a teflon stirbar was charged with $55.4 \mathrm{mg}(0.255 \mathrm{mmol})$ of benzyl-protected coniine and $11.1 \mathrm{mg}$ of $\mathrm{Pd}(\mathrm{OH})_{2}$ on carbon ( $20 \%$ by wt) in $1.6 \mathrm{~mL}$ EtOH (distilled from $\mathrm{CaH}_{2}$ ). The container was placed inside high pressure apparatus, which was subsequently sealed and pressurized to $400 \mathrm{psi}_{2}$. After stirring for $48 \mathrm{~h}$ (TLC analysis after $24 \mathrm{~h}$ indicated that starting material remained), the reaction vessel was removed from the bomb and the solution was filtered through celite (MeOH used to complete the transfer). After concentration to $\sim 100 \mu \mathrm{L}$ with a gentle purge of $\mathrm{N}_{2}$, the mixture was purified by silica gel chromatography in 10:1 $\mathrm{CH}_{2} \mathrm{Cl}_{2}: \mathrm{MeOH}$ washed with $2 \% \mathrm{v} / \mathrm{v}$ concentrated $\mathrm{NH}_{4} \mathrm{OH}$ to give coniine $(27.5 \mathrm{mg}$, $0.216 \mathrm{mmol}$ ) in 84\% yield. IR (neat): $3277(\mathrm{w}), 2937(\mathrm{~s}), 2851(\mathrm{~s}), 2796(\mathrm{~m}), 1443(\mathrm{~m})$, $1332(\mathrm{~m}), 1128(\mathrm{~m}), 751(\mathrm{~m}) \mathrm{cm}^{-1}$; ${ }^{1} \mathrm{H}$ NMR (400 MHz, $\mathrm{CDCl}_{3}$ ): $\delta 3.06$ (dddd, $J=11.7$, 4.0, 2.2, $\left.1.8 \mathrm{~Hz}, 1 \mathrm{H}, \mathrm{NCH}_{\mathrm{A}} \mathrm{H}_{\mathrm{B}}\right), 2.61\left(\mathrm{ddd}, J=11.7,11.7,2.6 \mathrm{~Hz}, 1 \mathrm{H}, \mathrm{NCH}_{\mathrm{A}} \mathbf{H}_{\mathbf{B}}\right), 2.47-$ $2.41\left(\mathrm{~m}, 1 \mathrm{H}, \mathrm{NCHR}_{2}\right), 1.78-1.01(\mathrm{~m}, 11 \mathrm{H}), 0.90\left(\mathrm{dd}, J=7.0,7.0 \mathrm{~Hz}, 3 \mathrm{H}, \mathrm{CH}_{3}\right) ;{ }^{13} \mathrm{C}$ NMR (100 MHz, $\left.\mathrm{CDCl}_{3}\right): \delta 56.8,47.4,39.8,33.1,26.8,25.1,19.2,14.4$; HRMS Calcd for $\mathrm{C}_{8} \mathrm{H}_{17} \mathrm{~N}(\mathrm{M}+\mathrm{H}): 128.1439$, Found: $128.1434 ;[\alpha]_{\mathrm{D}}-5.5\left(c=1, \mathrm{CHCl}_{3}\right)$, literature ${ }^{22}$ $[\alpha]_{\mathrm{D}}^{20}-8.1\left(c=2, \mathrm{CHCl}_{3}\right)$.

(22) Moody, C. J.; Lightfoot, A. P.; Gallagher, P. T. J. Org. Chem. 1997, 62, 746-748. 